\title{
Optically Detected Magnetic Resonance Studies on $\pi$-conjugated semiconductor systems
}

By

\author{
Ying Chen \\ A dissertation submitted to the graduate faculty \\ in partial fulfillment of the requirement for the degree of \\ DOCTOR OF PHILOSOPHY
}

Major: Condensed Matter Physics

Program of Study Committee:
Joseph Shinar, Major Professor
Adam Kaminski
Vladimir Kogan
Curtis Struck
Gary Tuttle

Iowa State University

Ames, Iowa

2011

Copyright $($ Ying Chen, 2011. All rights reserved. 


\section{TABLE OF CONTENTS}

1. Introduction to $\pi$-conjugated organic materials 1

Abstract 1

$1.1 \pi$-conjugated materials $\quad 1$

1.2 Applications of organic semiconductors 5

1.2.1 OLEDs 5

$\begin{array}{ll}1.2 .2 \text { OSCs } & 10\end{array}$

$\begin{array}{ll}\text { 1.2.3 Other applications } & 11\end{array}$

$\begin{array}{ll}1.3 \text { Conclusion } & 12\end{array}$

$\begin{array}{ll}\text { References } & 12\end{array}$

2. Introduction to Optically Detected Magnetic Resonance

$\begin{array}{ll}\text { (ODMR) } & 15\end{array}$

$\begin{array}{ll}\text { Abstract } & 15\end{array}$

$\begin{array}{ll}\text { 2.1 PL-detected magnetic resonance (PLDMR) } & 15\end{array}$

$\begin{array}{ll}\text { 2.1.1 Single modulated PLDMR (sm-PLDMR) } & 17\end{array}$

2.1.2 Double modulated PLDMR (DM-PLDMR) 19

$\begin{array}{ll}2.1 .3 \text { Triplet powder pattern } & 21\end{array}$

2.2 EL detected magnetic resonance (ELDMR) 24

2.3 Photocurrent detected magnetic resonance (PCDMR) 25

2.4 Photoinduced absorption detected magnetic resonance (PADMR) 26 
References

3. ELDMR studies in Alq3 and Rubrene doped Alq3 OLEDs

Abstract

3.1 Degradation measured by ELDMR in Alq3 OLEDs

3.1.1 Introduction

3.1.2 Experimental

3.1.3 Results and discussion

3.1.4 Conclusion

3.2 ELDMR in Rubrene doped Alq3 devices

3.2.1 Introduction

3.2.2 Experimental

3.2.3 Results and discussion

3.2.4 Conclusion

References

4. PLDMR Studies on P3HT and MEH-PPV films

Abstract

4.1 Double modulated PLDMR studies in P3HT and MEH-PPV films

4.1.1 Introduction

4.1.2 Experimental 52

4.1.3 Results and discussion

4.1.4 Conclusion 
4.2.1 Introduction

4.2.2 Quantitative analysis based on TPQ model

4.2.3 Conclusion

66

References

66

5. Colossal transient room temperature photocurrent-detected magnetic resonance in organic devices: a magnetic resonance-induced switch

Abstract 68

5.1 Introduction 68

5.2 Experimental 69

5.3 Results and discussions 70

5.3.1 Colossal PCDMR 70

5.3.2 Possible mechanisms 75

5.3.3 A quantitative analysis 81

5.4 Conclusions 84

References

6. Evidence for holes beyond the recombination zone \& trions in the electron transport layer of OLEDs

Abstract

6.1 Introduction 88

6.2 Experimental

6.2.1 ODMR and EDMR measurements. 
6.2.2 OLED Fabrication

6.2.3. Transient EL measurements.

6.3 Results and discussions

6.3.1 ELDMR

6.3.2 Transient EL

96

6.4 Conclusions

99

References

99

Summary and general conclusion

102

Acknowledgements

104 


\title{
Chapter 1. Introduction to $\pi$-conjugated organic materials
}

\begin{abstract}
Since the early $80 \mathrm{~s}, \pi$-conjugated semiconducting materials have attracted steadily growing interest from both academia and industry, and in particular during the past decade for applications such as organic solar cells (OSCs), organic light emitting diodes (OLEDs) and organic field effect transistors (OFETs) [1-5]. They have several advantages over inorganic semiconductors such as flexibility, lower manufacturing costs, color tunability in display and lighting applications, and potential for large active area applications. For all these applications, understanding of device physics such as exciton dynamics and carrier transport is the key for further developments. In this chapter, some basic information on $\pi$-conjugated organic semiconductors is provided. Applications of these organic semiconductors are briefly introduced. A deeper understanding of the physical processes, especially those under operational situations is required to help further development of organic semiconductors.
\end{abstract}

\section{1. $\pi$-conjugated materials}

As the result of mixing $2 s$ and $2 p$ orbitals in the carbon atom, it is possible for these orbitals to combine to form three planar $\mathrm{sp}^{2}$ orbitals ( $\mathrm{sp}^{2}$ hybridization). One of the three original $\mathrm{p}$ orbitals $\left(\mathrm{p}_{\mathrm{z}}\right)$ remains unaltered, while the $2 \mathrm{~s}$ orbital mixes with the other two p orbitals. The bonds formed from hybrid orbital in this case are called $\sigma$-bonds; The $\mathrm{p}_{\mathrm{z}}$ orbital is perpendicular to the plane of $\mathrm{sp}^{2}$ hybridization. In organic materials, for example, benzene and anthracene, the $\mathrm{p}_{z}$ orbitals of neighboring carbon 
atoms overlap with each other, together creating what is called a $\pi$-bond. Electrons are delocalized in $\pi$-bond. The structures of several such $\pi$-conjugated materials used in this work are shown in Figure 1.1.

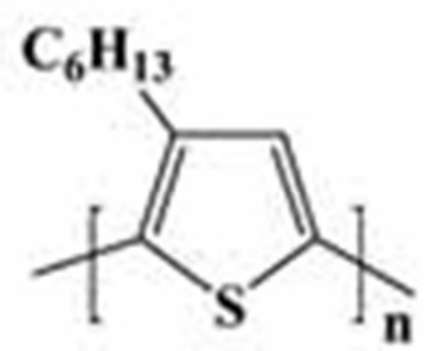

poly[2-methoxy-5-(2 -ethyl-hexyloxy)-1,4-phenyl ene vinylene] (MEH-PPV)

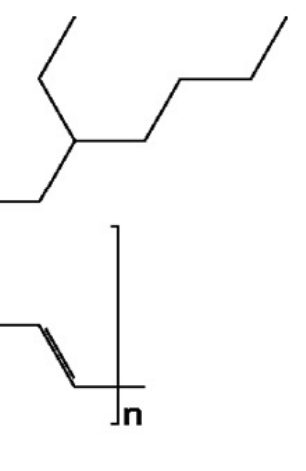

poly(3-hexylthiophene) (P3HT)

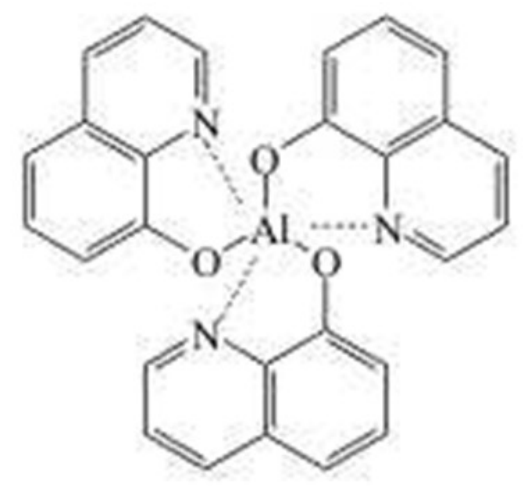

Tris(8-hydroxyquinolinato)aluminium $\left(\mathrm{Alq}_{3}\right)$
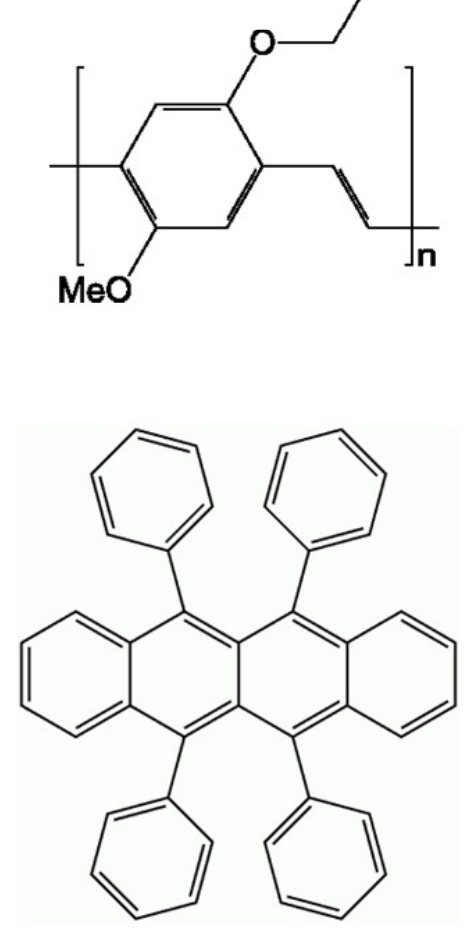

5,6,11,12-tetraphenylnaphthacene (Rubrene)

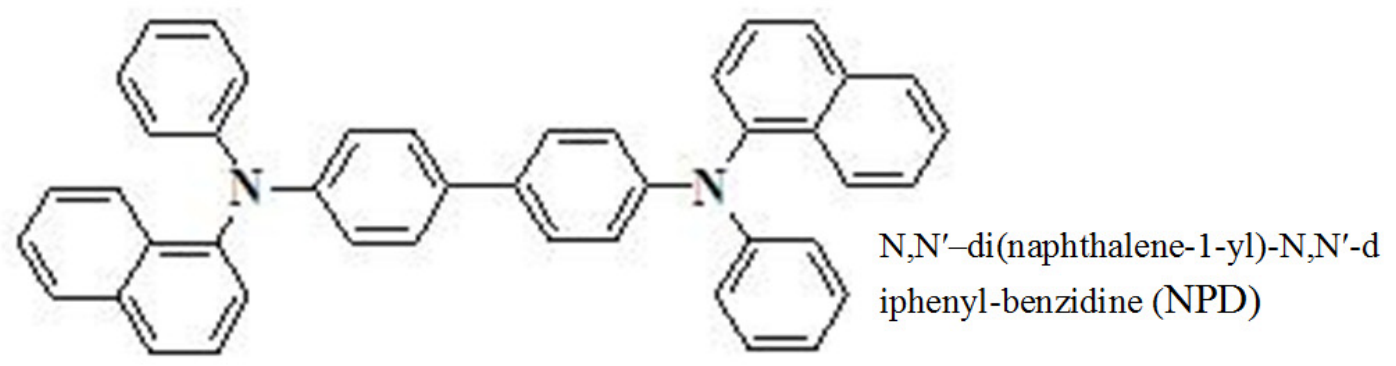

Figure 1.1, Molecular structures for several organic materials

In a $\pi$-conjugated molecule or polymer, the electrons occupy the molecular orbitals up to the highest occupied $(\pi)$ molecular orbital (HOMO) and there is a gap between that 
and the lowest unoccupied antibonding $\left(\pi^{*}\right)$ molecular orbital (LUMO), as shown in Figure 1.2. This gap between the LUMO and HOMO levels is typically in the range of $1.5-3 \mathrm{eV}$ [6], resulting in semiconducting properties. Under photoexcitation, an electron jumps from an orbital at or below the HOMO to an orbital at or beyond the LUMO, leaving a hole in the HOMO and thus forming an exciton, i.e., a Coulomb bound electron-hole pair. After the excitation, various processes may occur: (i) After a quick process of thermalization ( $\sim 100 \mathrm{fs}$ ) [6], the exciton relaxes to the lowest excited state. From there, the singlet exciton (SE) will decay to the ground state, either radiatively or nonradiatively. (ii) The excited SE may convert to a triplet exiton (TE) through intersystem crossing (ISC). This process is extremely important in phosphorescent materials in which the spin-orbit coupling induced by heavy metal atoms greatly enhances the ISC and allows radiative decay from the TE to the ground state. (iii) The SEs may also dissociate to form polarons through charge transfer, as shown in Figure 1.3. A polaron is a charge that interacts with surrounding lattice polarization and deformation.

Exciton dissociation is the basis for photovoltaic applications. In the case of current injection, the process is in the other way: charge carriers form polarons on the active molecules. Two polarons may pair together to form either TEs or SEs. Without any difference in generation cross sections, the ratio for TE and SE should be 3:1. However, there are arguments that the SE formation is more favored in some material $[7,8]$. 


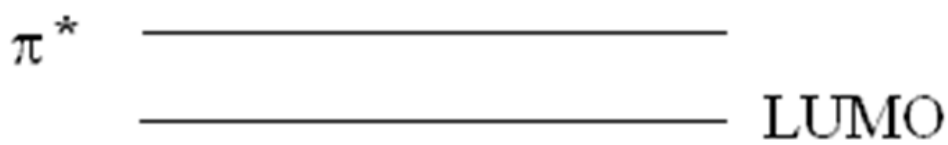

$\pi$

HOMO

Figure 1.2 HOMO and LUMO in conjugated material

Triplet Excitons

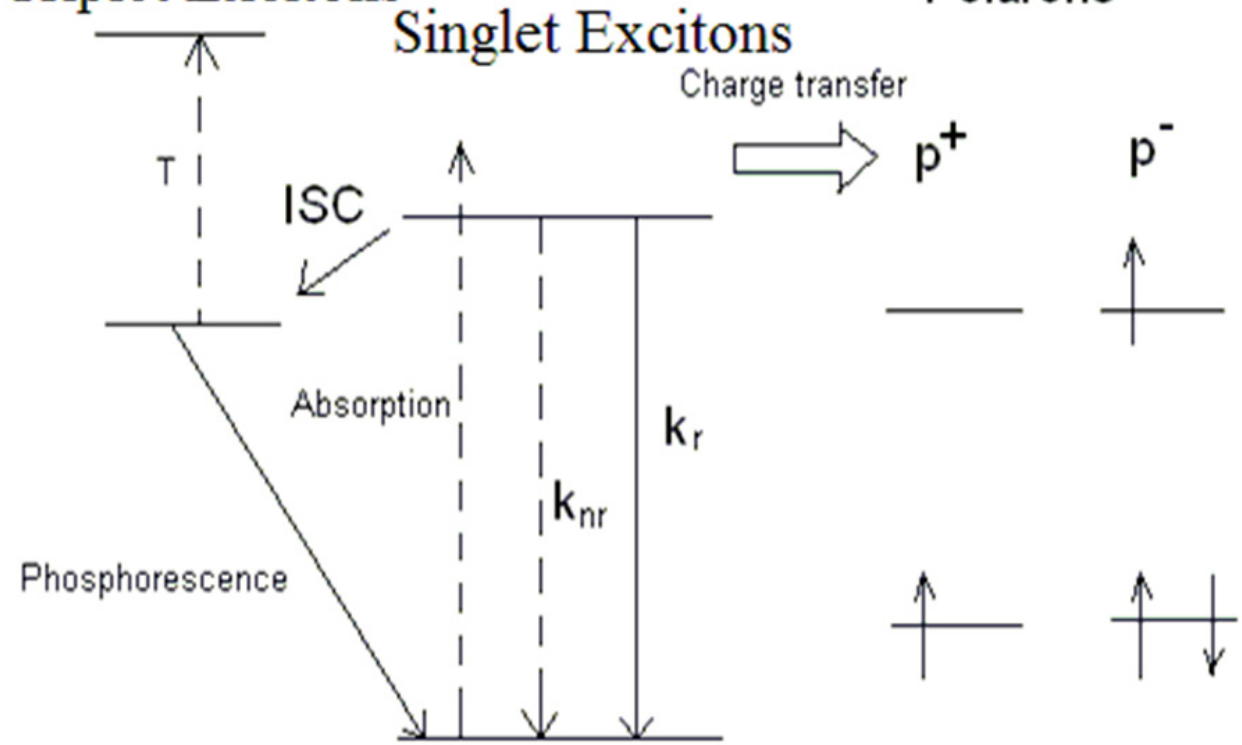

\section{Ground State}

Figure 1.3 Photoexcitation and various processes following the excitation [6].

Besides the processes mentioned above, there are also luminescence quenching processes that are significant in $\pi$-conjugated materials:

(a) SEs get quenched by TEs 
$S+T \rightarrow T^{*}+$ phonons

where $S$ represents SE, $T$ represents TE, and $T^{*}$ represents a TE with higher energy.

(b) SEs get quenched by polarons

This includes the cases of either SEs directly annihilated by the polaron,

$$
S+p^{+/-} \rightarrow p^{+/-^{*}}+\text { phonons }
$$

(where $p^{+/-}$represents polaron that is positively charged or negatively charged and $p^{+/-^{*}}$ represents polaron with higher energy), or the polaron induces dissociation of the SE

$$
S+p^{+/-} \rightarrow p^{+}+p^{-}+p^{+/-}+\text {phonons }
$$

(c) SEs get quenched by bipolarons.

Bipolarons are doubly charged polarons that are either positively charged $b p^{++}$or negatively charged $b p^{--}$. Similar to the case for polarons, there are two quenching mechanisms for this process

$$
\begin{aligned}
& S+b p^{++/--} \rightarrow b{p^{++/--^{*}}+\text { phonons }}^{S+b p^{++/--} \rightarrow p^{+}+p^{-}+b p^{++/--}+\text {phonons }}
\end{aligned}
$$

(d) TEs get quenched by polarons.

$$
T+p \rightarrow p^{*}+\text { phonons }
$$

As mentioned, in phosphorescent materials, TEs can decay radiatively to the ground state giving phosphorescence. So this process is very important for those materials.

\subsection{Applications of organic semiconductors}

\subsubsection{OLEDs}


In 1987, Tang and VanSlyke described multilayered OLEDs based on organic small molecule films sandwiched between electrodes [9]. Semiconducting conjugated polymers were also found to emit green-yellow light under an electric field in 1990 [10]. Since then, great efforts have been made to develop OLEDs. The commercialization of OLEDs was realized in 1998. They are now poised to become the next generation of flat panel displays because of their advantages in comparison to liquid crystal displays and white OLEDs (WOLEDs) may attain a dominant position in solid state lighting applications.

In OLEDs, holes and electrons are injected through the electrodes. After transport in the organic material, they recombine to form SEs and TEs and then emit light through radiative decay. OLEDs usually have heterojunction multilayer structures, especially in small molecular material based OLEDs (SMOLEDs). Due to the different mobilities of electrons $\left(\mu_{e}\right)$ and holes $\left(\mu_{h}\right)$ (where usually $\mu_{e} / \mu_{h}<0.01$ ), the recombination zone in single layer devices is very close to the cathode and thus leads to electroluminescence (EL) quenching by direct electrode recombination and metallic mirror effect. Multilayer structures are used to avoid this problem. The recombination zone in multilayer devices is located at the heterojunction which is far from the electrodes, thus avoiding quenching by the electrodes. To further improve the efficiency and lifetime of the device, the structure of an OLED could be complex and include many layers between the electrodes, such as hole injecting layer, hole transport layer, electron blocking layer, active layer, hole blocking layer, electron transport layer, electron injecting layer. . Usually indium tin oxide (ITO) is used as the 
transparent anode and $\mathrm{Al}$ is used as the cathode. A typical such structure is shown in Figure 1.4.

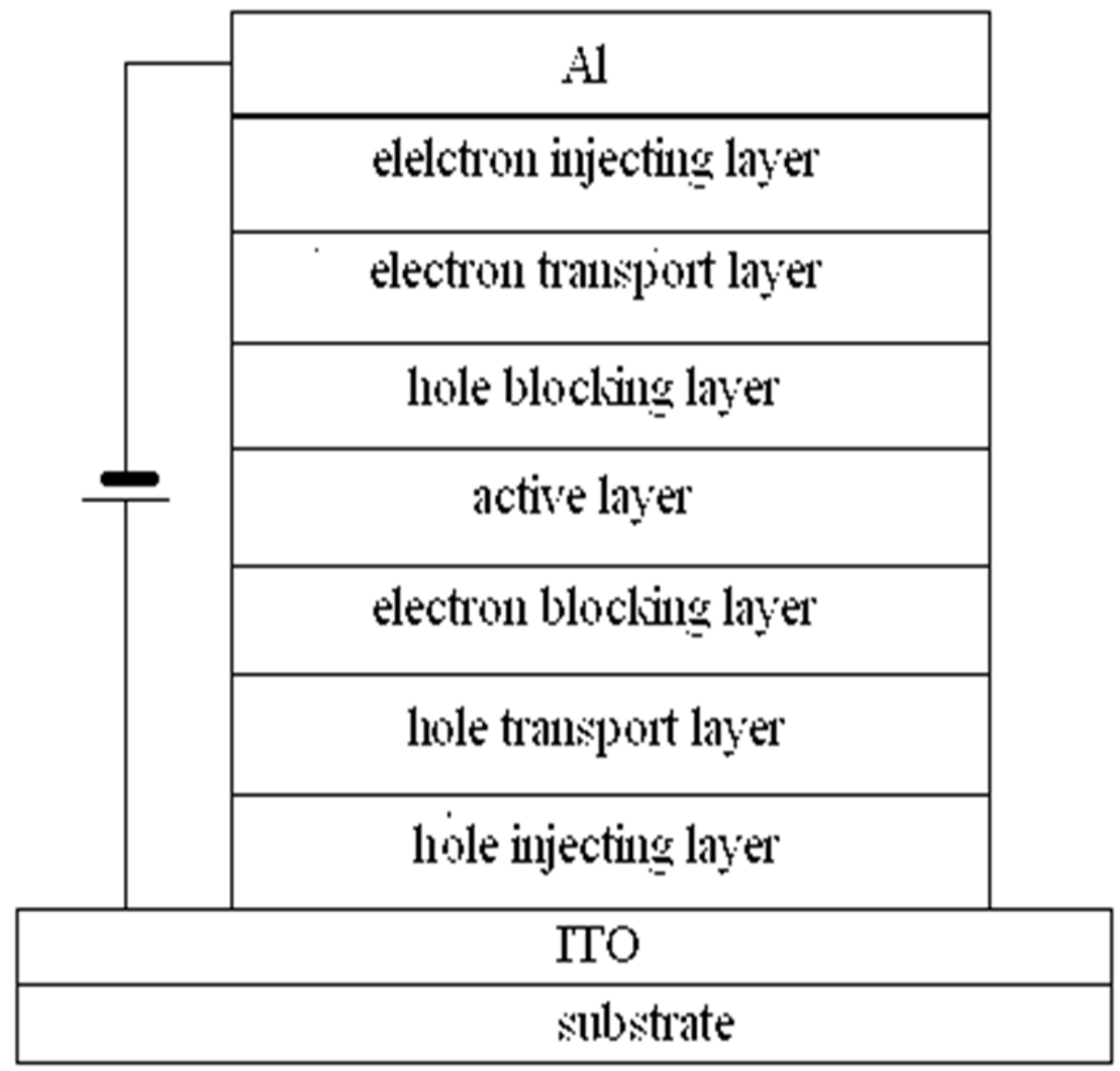

Figure 1.4 A multilayer OLED

For OLED applications, crucial factors are high efficiency and long lifetime. The external quantum efficiency of an OLED $\eta_{E L}{ }^{\text {ext }}$ is composed of several components, as shown in Equation 1.7:

$\eta_{E L}{ }^{e x t}=\eta_{O C} \gamma \eta_{e x c} \phi_{P L}$

where $\eta_{O C}$ is the outcoupling factor which is only $\sim 20 \%$ if $\eta_{O C}=1 /\left(2 n^{2}\right)(n \sim 1.7$ is the refractive index of the organic material), $\gamma$ is the carrier balance factor, $\eta_{e x c}$ is the fraction of excitons formed by carrier recombination that decay radiatively, and 
$\phi_{P L}$ is the emitting material's photoluminescence (PL) quantum yield. The outcoupling $\eta_{O C}$ can be strongly enhanced through methods such as introducing micro structure to the other side of substrates [11] and replacing ITO with PEDOT:PSS to reduce the loss due to wave guiding [12]. Charge carrier balance can be improved through carefully selecting proper materials for each functional layer and controlling the structure parameters such as thickness. The value of $\eta_{e x c}$ is $25 \%$ for fluorescent emitter, since only the radiative decay of singlet excitons (SEs) generates EL, thus limiting $\eta_{E L}{ }^{e x t}$ to $\sim 5 \%$ taking into account the outcoupling factor. However, in phosphorescent emitters, SEs and TEs are mixed due to strong spin-orbit coupling, resulting in $\eta_{\text {exc }} \sim 100 \%$. Great efforts have also been done to find materials that have $\phi_{P L} \sim 100 \%$. However, due to the complexity of processes that go on in OLEDs during operation, a lot of work is still to be done to better understand various quenching mechanisms of luminescence.

Lifetime of OLED, or degradation, is another factor that is key to successful commercial applications. Organic materials are known to be unstable under ambient conditions. Exposure of devices to such conditions leads to cathode degradation and generation of non-emissive areas commonly known as dark spots. Since the late 1990s, after numerous studies were devoted to this subject [13-15], encapsulation methods were developed to eliminate the growth of dark spots resulting from interaction with air. Once the encapsulation problem was solved, the more challenging problem was to identify the intrinsic degradation mechanisms. Thus luminescence quenching processes and other physical processes required reexamination. Four 
aspects have been studied intensively in recent years:

(i) Thermal instability was proposed to be responsible for intrinsic degradation [16-19]. Especially in cases of short lifetime, low-glass-transition-temperature (low-Tg) used as hole transport materials (HTMs) were suggested to cause degradation. However, later studies showed that although high-Tg materials are still needed for applications at high temperature, they are not necessarily required for good operational stability at room temperature [20].

(ii) Trap formation during device operation plays a big role in intrinsic degradation. In particular, in polymer LEDs (PLEDs) such as those based on poly(phenylene vinylene) (PPV), the formation of bulk traps appears to be the main mechanism of device degradation [21]. On the other hand, degradation in SMOLEDs was proposed to be more related to formation of luminescence quenchers during operation [22].

(iii) Interfaces, especially in SMOLEDs, could be charge barriers and thus lead to degradation. Multiple studies have shown that by removing the abrupt interface of hole transport layer (HTL) and electron transport layer (ETL) through mixing of the two materials to form a emissive layer, the lifetime of the device can be greatly enhanced [23-25].

(iv) Anode degradation is another possible mechanism to explain intrinsic degradation. Since ITO is widely used as the anode for most OLEDs, the diffusion of indium or oxygen from ITO into the organic layers was investigated and found to be related to degradation [26, 27]. Inserting a layer of PEDOT:PSS, which stops the 
diffusion, was found to be effective in improving device lifetime.

\subsubsection{OSCS}

With the world energy crisis foreseeable, solar energy is proposed as one of the major solutions, which is renewable and expected to reduce the risk of global warming through reducing the emission of $\mathrm{CO}_{2}$ [28]. Solution processed bulk-heterojunction polymer photovoltaic cells were first reported in 1995 [29]. Since then, OSCs have been increasingly attractive because of their ease of processing, mechanical flexibility and potential for low cost large area printing [30] studies on OSC systems such as the mixture of poly(3-hexylthiophene):1-(3-methoxycarbonyl)propyl-1-phenyl[6,6]C61(P3HT:

PCBM) have generated numerous publications [31].

To quantify the performance of a solar cell, there are several parameters. Among them, the ratio of maximum power output and power of incident light $\mathrm{P}_{0}$ gives the energy (or power) conversion efficiency of the cell $\eta=\frac{P_{\max }}{P_{0}}$

Another important parameter is external quantum efficiency (EQE) which is defined as the ratio of collected electrons to the incident photons. The quantum conversion efficiency of real solar cell is usually much lower than $100 \%$, due to losses associated with reflection of incident photons, their imperfect absorption by the photovoltaic material and recombination of the charge carries before they reach the electrodes. There are also electrical resistance losses in the cell and in the external circuit. Pure 
conjugated polymer photovoltaic cells only have energy conversion efficiency typically $\sim 0.01 \%$ [32] due to the fast recombination of the carriers to excitons. Therefore, heterojunction structures where an electron acceptor is added to help exciton dissociation is key to improving the efficiency. The overall process occurring in the hybrid polymer may be divided into several steps [33]. Correspondingly, the EQE can be written as:

$E Q E=\eta_{A} \eta_{\text {diff }} \eta_{\text {sep }} \eta_{t r} \eta_{c c}$

where $\eta_{A}$ is the photon absorption yield, $\eta_{\text {diff }}$ is the charge carriers diffusion yield, $\eta_{\text {sep }}$ is the charge carrier separation yield, $\eta_{t r}$ is the charge transport yield in donor and acceptor materials, and $\eta_{c c}$ is the charge collection yield. The crucial element for improvement of overall efficiency is the alignment of the energy levels of component materials and electrodes. As in OLEDs, stability/degradation is another important factor for successful application. When oxygen and water are present, their diffusion into the device is generally regarded as the dominant source of degradation. It was found that oxygen and water diffusion through the outer electrode is the main path of such degradation $[34,35]$. Photooxidation is another important factor that causes degradation, especially in PPV-based devices [36-38]. Besides these, chemical degradation of electrodes such a ITO, which was also mentioned in OLED degradation, is also proposed as a possible degradation mechanism for OSCs.

\subsubsection{Other applications}

Besides applications in OLEDs and OSCs, another important application is OFETs. OFETs are considered as the key component of organic integrated circuits for 
use in flexible smart cards, low cost radio frequency identification tags and organic active matrix displays, etc. [39-41]. A typical OFET is composed of a gate electrode, a gate dielectric layer, an organic semiconductor layer and source and drain electrodes. As in traditional FETs, current is injected from the source into the organic semiconductor layer and collected by the drain electrode. The conductance can thus be controlled by the gate voltage.

Organic semiconducting materials can also be used in spintronic applications, such as spin valves. It is reported that a strong spin valve effect of $\sim 22 \%$ was achieved in a tris(8-hydroxyquinolinato) aluminium ( $\mathrm{Alq}_{3}$ )-based device [42].

\subsection{Conclusion}

$\pi$-conjugated organic semiconductors are promising building blocks of next generation electronics. Great efforts have been devoted to this area. Some applications, such as OLEDs, are already commercialized. However, greater application demands a deeper understanding of physical processes in these materials and devices.

\section{References}

[1]. J. Shinar et al., J. Phys. D: Appl. Phys., (2008), 41, 133001

[2]. D. Braga et al., Adv. Mater., (2009), 21, 1473

[3]. G. Gelinck et al., Adv. Mater., (2010), 22, 3778

[4]. T. Ameri et al., Energy Environ. Sci., (2009), 2, 347

[5]. J. Clark et al., Nature Photonics, (2010), 4, 438

[6]. Organic Light Emitting Devices, edited by J. Shinar, (2004), Springer-Verlag New York, Inc. 
[7]. M. Wohlgenannt et al., Nature, (2001), 409, 494

[8]. M. Wohlgenannt et al., Phys. Rev. Lett., (2002), 88, 197401

[9]. C. W. Tang et al., Appl. Phys. Lett., (1987), 51, 913

[10]. J. H. Burroughes et al., Nature, (1990), 347, 539

[11]. R. Liu et al., Optics Express, (2011), 19, A1272

[12]. M. Cai et al., Appl. Phys. Lett., (2011), 99, 153303

[13].W. Bijnens et al., Synth. Met., (1996), 83, 261

[14].Y. F. Liew et al., Appl. Phys. Lett., (2000), 77, 2650

[15].S. F. Lim et al., Appl. Phys. Lett., (2001), 78, 2116

[16].N. A. Lyengar et al., Macromolecules, (2003), 36, 8976

[17].Q. Peng et al., Macromolecules, (2004), 37, 260

[18].H. Murata et al., Appl. Phys. Lett., (1999), 75, 3252

[19].K. Okumoto et al., Synth. Met., (2001), 121, 1655

[20].C. Adachi et al., Appl. Phys. Lett., (1995), 66, 2679

[21].S. Kim et al., J. Soc. Inf. Disp., (2004), 5, 14

[22].H. Aziz et al., Science, (1999), 283, 1900

[23].V. E. Choong et al., Appl. Phys. Lett., (1999), 75, 172

[24].Z. D. Popovic et al., Proc. SPIE, (1999), 683467

[25].A. B. Chwang et al., Appl. Phys. Lett., (2002), 80, 725

[26].T. W. Lee et al., Appl. Phys. Lett., (2006), 89, 123505

[27].M. P. Dejong et al., Appl. Phys. Lett., (2000), 77, 2255

[28].Q. Shiermier et al., Nature, (2008), 454, 816 
[29].G. Yu et al., Science, (1995), 270, 1789

[30].C. J. Brabec et al., Adv. Funct. Mater., (2001), 11, 15

[31].P. W. Blorn et al., Adv. Mater., (2007), 19, 1551

[32].G. Yu et al., Appl. Phys. Lett., (1994), 64, 1540

[33].B. R. Saunders et al., Adv. Colloid Interface Sci., (2008), 138, 1

[34].M. Lira-Cantu et al., Chem. Mater., (2006), 18, 5684

[35].F. C. Krebs et al., Prog. Photovol. Res. Appl., (2007), 15, 697

[36].N. Dam et al., Chem. Mater., (1999), 11, 1302

[37].R. F. Bianchi et al., J. Polym. Sci. Part B: Polym. Phys., (2004), 42, 1033

[38].J. Alstrup et al., Sol. Energy Mater. Sol. Cells, (2007), 91, 394

[39].B. Crone et al., Nature, (2000), 403, 521

[40].P. F. Baude et al., Appl. Phys. Lett., (2003), 82, 3964

[41].Y. Guo et al., Adv. Mater., (2010), 22, 4427

[42].M. Prezioso et al., Adv. Mater., (2011), 23, 1371 


\title{
Chapter 2. Introduction to Optically Detected Magnetic Resonance (ODMR)
}

\begin{abstract}
Developed from electron-spin resonance (ESR) techniques, since the 1960s ODMR has been widely used to study carriers dynamics in various systems such as inorganic semiconductors and organic semiconductors $[1,2]$. In this technique, optical properties of the sample under investigation are monitored, while transitions between sublevels of spin non-zero species are induced by resonance conditions. This method is very sensitive so that it is reported to be able to, e.g., detect a single spin on tetracene [3]. Common optical properties monitored in this method are photoluminescence (PL), photoinduced absorption (PA), electroluminescence (EL), photoconductivity (PC) and so on. In this part, a brief introduction to these techniques is given.
\end{abstract}

\subsection{PL-detected magnetic resonance (PLDMR)}

In ODMR measurements, resonance condition is fulfilled when the microwave photon energy matches the splitting in energy levels of the species under investigation.

$h v_{\mu w}=g \beta H \Delta m_{S}$

where $g$ is the $g$-value of the spin-nonzero species, $\beta$ is the Bonr magneton, $H$ is the applied magnetic field, and $\Delta m_{S}$ is the spin difference between energy sublevels. Resonance induces a population mixing between these sublevels. If off-resonance populations of these sublevels are different, this mixing effect will lead to population 
changes under resonance. If the photoluminescence comes from the radiative decay of SEs, then since SEs have spin $=0$, no direct effect will be observed. However, as mentioned in the previous chapter, luminescence can be quenched or enhanced by various non-zero spin species, such as TEs and polarons. Thus, by changing the sublevel populations of these species, the resonance may induce a change in the total population if different spin states have different decay rate. And this will in turn induce a change in PL.

It should be noted that besides the quenching processes (a)-(d) listed in Chapter 1, there are also many other processes that could affect the PL, such as:

(e). polarons recombine to form SEs

$$
p^{-}+p^{+} \rightarrow S
$$

(f). polarons recombine to form TEs

$$
p^{-}+p^{+} \rightarrow T
$$

(g). polarons annihilate each other

$$
p^{-}+p^{+} \rightarrow S_{0}+\text { phonon }
$$

(h). Two polarons combine to form bipolarons (bps)

$$
p^{-/+}+p^{-/+} \rightarrow b p^{--/++}
$$

(i). Bipolarons combine with polarons to form polarons with opposite charge.

$$
b p^{++/--}+p^{-/+} \rightarrow p^{+/-}
$$

(j). Triplets annihilation generates singlets

$$
T+T \rightarrow S+\text { phonon }
$$

$S_{0}$ represents the ground state. It should be noted that although some of these 
processes do not directly involve SEs, since the species involved are SE quenchers or generators, they can affect SE population indirectly

All these processes can happen simultaneously in the material. Further more, the combination of these processes can be very different in different systems. Thus multiple experiments are usually needed to nail down the mechanism for an observed resonance.

\subsubsection{Single modulated PLDMR (sm-PLDMR)}

A typical experiment setup for sm-PLDMR is shown in Figure 2.1

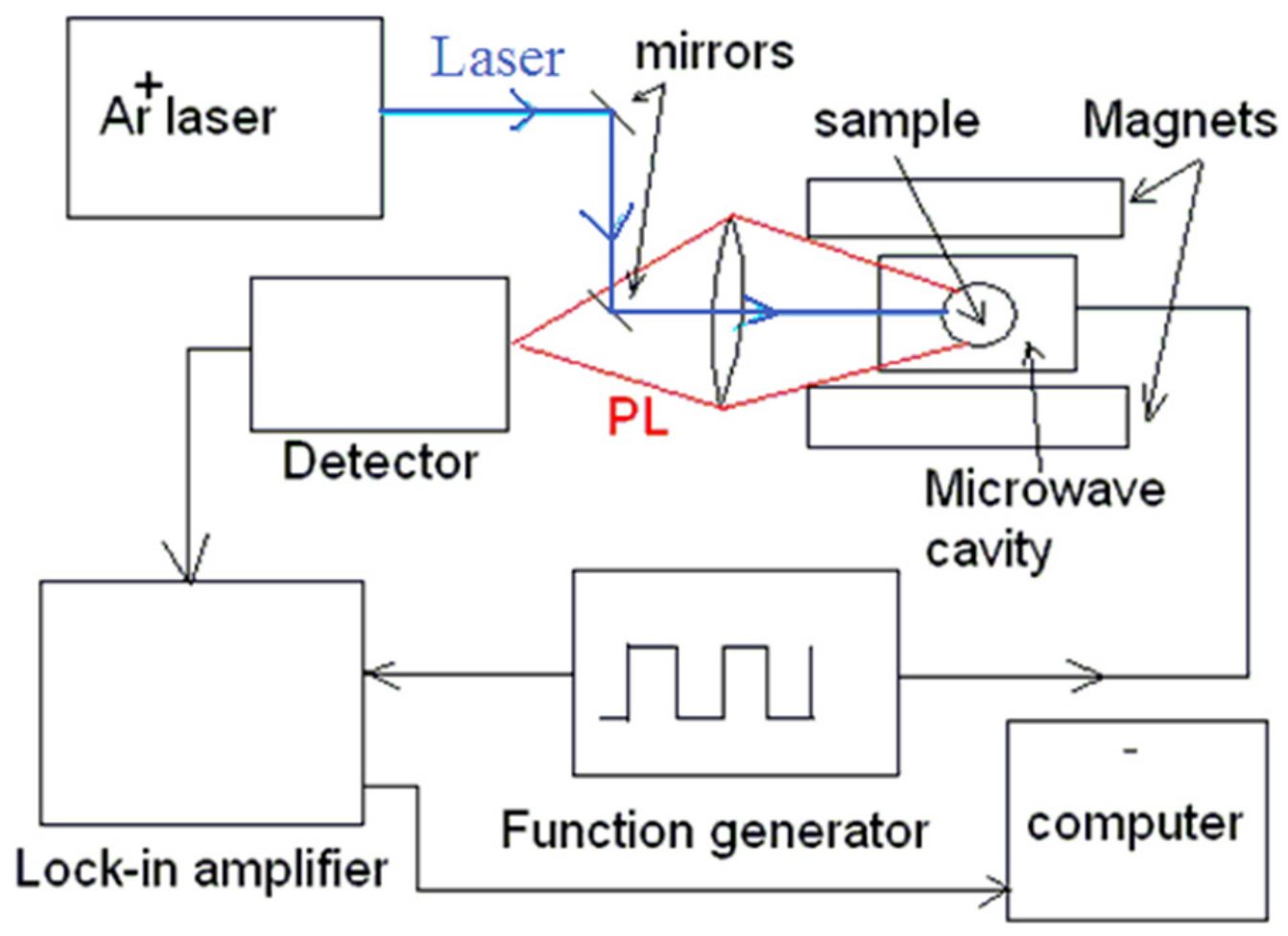

Figure 2.1 Schematic representation of the PLDMR system

The sample is located in a cryostat in the microwave cavity. It is excited by the $\mathrm{Ar}^{+}$ 
laser in a CW mode. In all of the experiments shown in this work, we used the $488 \mathrm{~nm}$ blue laser line. A function generator gives chopping signal with $f=f_{M}$ to the microwave switch so that the microwave power is modulated accordingly, and simultaneously gives the reference signal to the lock-in amplifier. The PL is then collected by the detector and transferred to the lock-in amplifier. The amplifier sends the modulation amplitude of the PL to the computer. During measurements, the magnetic field $H$ sweeps through a certain range. When the splitting of sublevels matches the microwave photon energy, resonance can be seen.

Take the spin-1/2 PLDMR in poly[2-methoxy-5-(2-ethylhexyloxy)1,4-phenylenevinylene] (MEH-PPV) for example: According to triplet-polaron quenching model $[4,5]$, on resonance, the triplet population that could quench the SEs is reduced through enhanced TE- $p$ quenching (process (d) in chapter 1). Thus the luminescence is enhanced under resonance conditions. A result is shown in Figure 2.2.

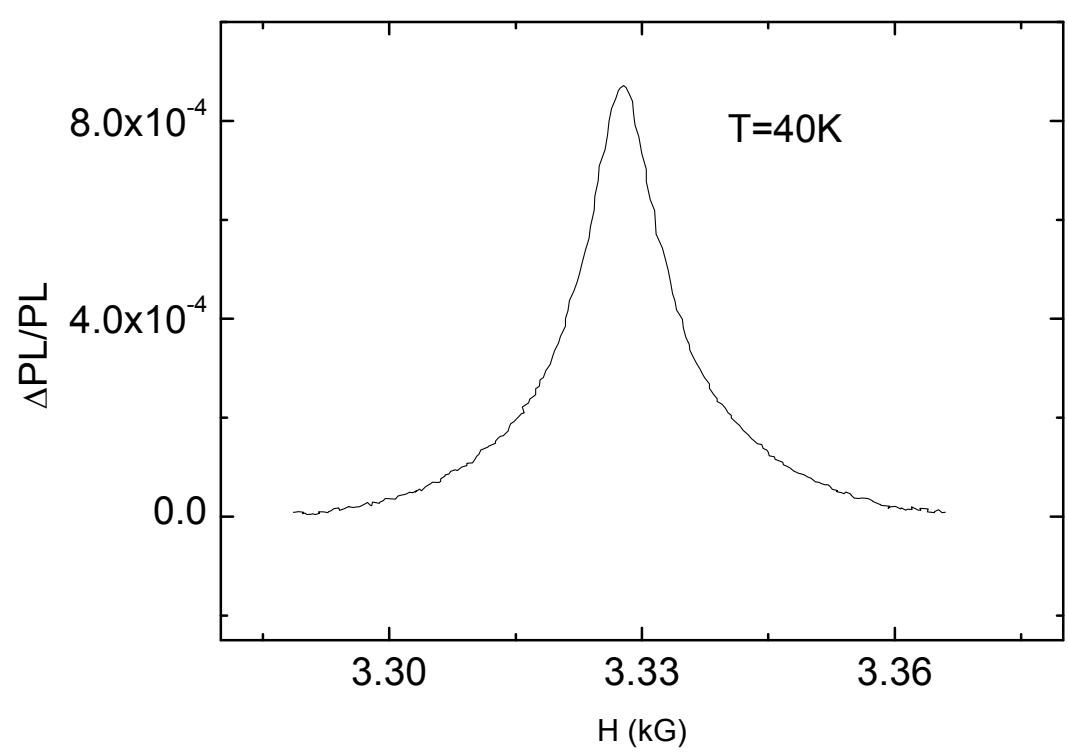

Figure 2.2 The positive spin-1/2 PLDMR in an MEH-PPV film 
According to equation 2.1, the g value for this resonance can be calculated

$$
g=\frac{h v}{\beta H}
$$

which gives $g=2.002(1)$ here. Note in this spin-1/2 resonance, $\Delta m_{S}= \pm 1$.

Other resonances, such as the triplet powder pattern $\Delta m_{S}= \pm 1$ and $\Delta m_{S}= \pm 2$ of the same film, can provide other information about this material.

Besides the information directly obtained from one resonance spectrum, richer information can be obtained by studying the behavior of each resonance with varying microwave power, modulation frequency, excitation intensity, etc.

\subsubsection{Double modulated PLDMR (DM-PLDMR)}

Figure 2.3 shows the experimental setup for DM-PLDMR

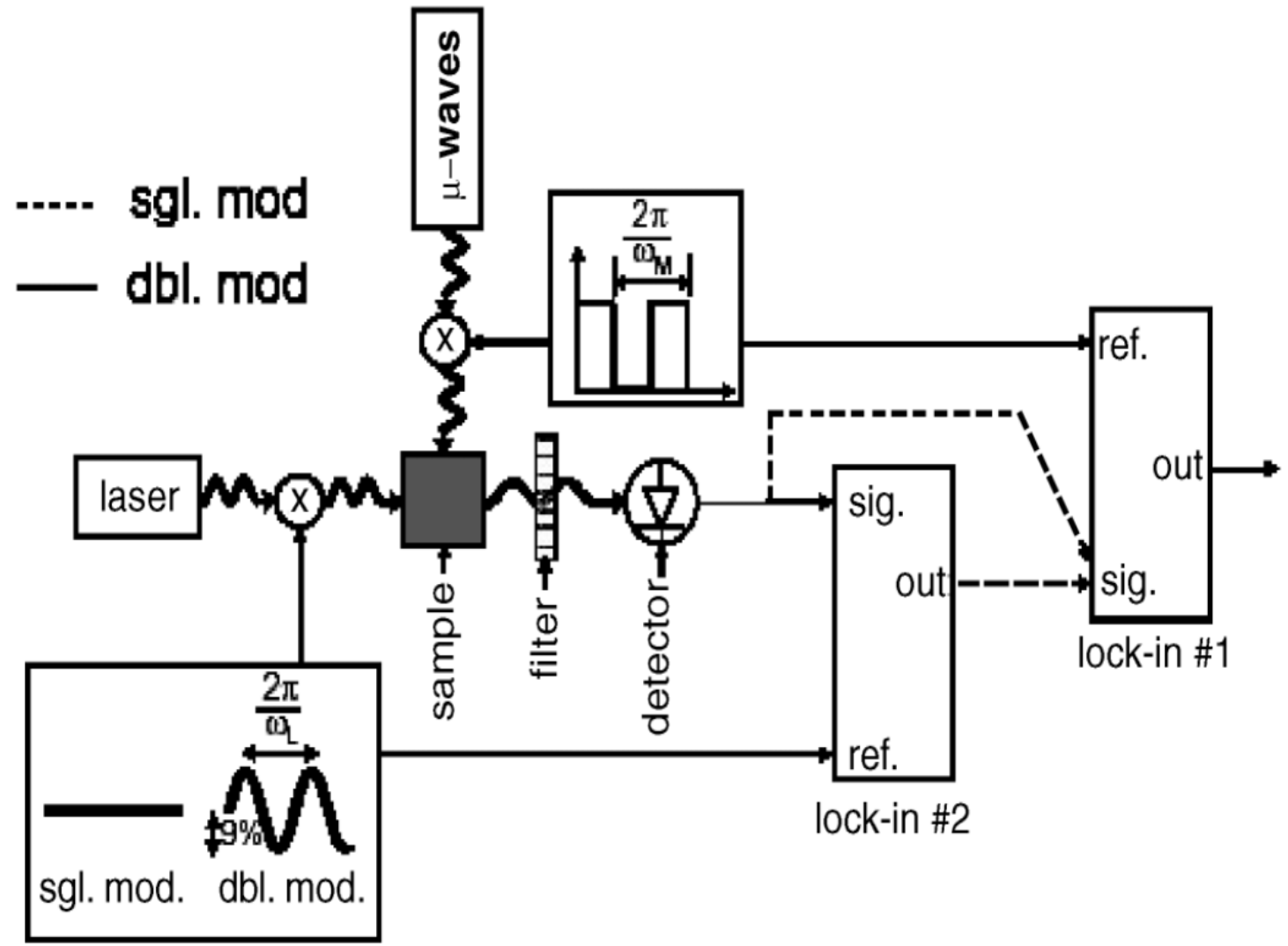

Figure 2.3 Schematic representation of the PLDMR system. [4] 
In DM-PLDMR, both the microwave power and the excitation power are modulated, which gives the name "double modulation". Two lock-in amplifiers are used in this experiment. To ensure the signal is not distorted due to the filtering function of the amplifiers, the time constant set on the amplifier \#2 must be small to minimize the side band attenuation of amplifier \#1. In the work of Lee et al. $[4,5]$ it was shown that the frequency dependence of the DM-PLDMR exclusively favors the quenching model mentioned above. The curve can not be explained by a spin-dependent polaron recombination model (SDR model) $[6,7]$ as shown in Figure 2.4 .

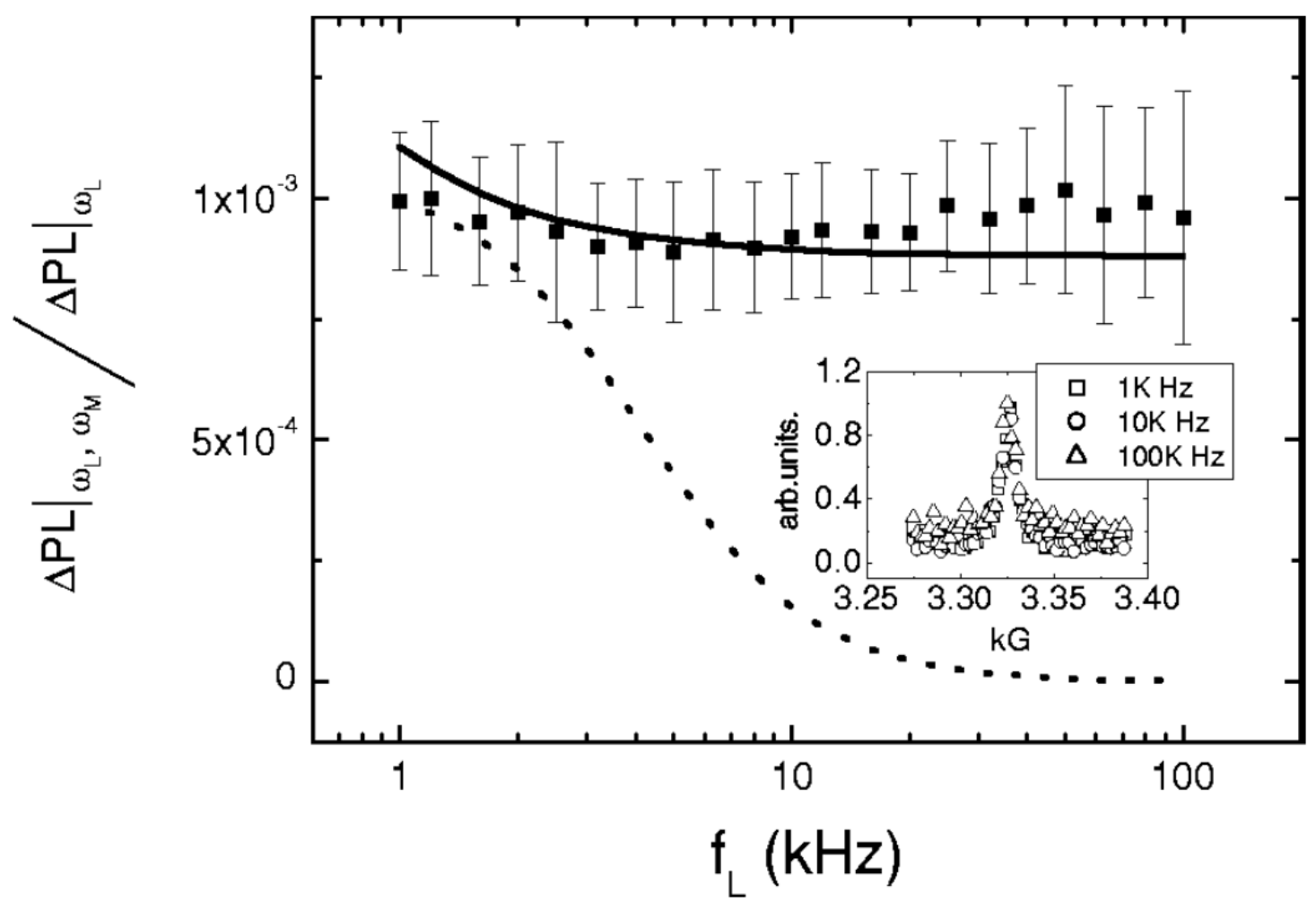

Figure 2.4 Frequency dependence of DM-PLDMR amplitude. Dotted line is the simulation based on SDR model [5]. 
However, this experiment did not rest the case. Supporters of SDR model responded by pointing out that the quenching model can not explain the sm-PLDMR well enough if one takes a close look at the in-phase and quadrature components [7-9]. At one point, it appears that the SDR model cannot explain the DM-PLDMR result while quenching model cannot explain the sm-PLDMR. However, as will be shown in chapter 4, the dilemma can be solved if one takes account of the fact that the quenching model actually is a two-step process, which requires careful treatment when dealing with the details of the signal.

\subsubsection{Triplet powder pattern}

The splitting of the sublevels of TEs is shown in figure 2.5.

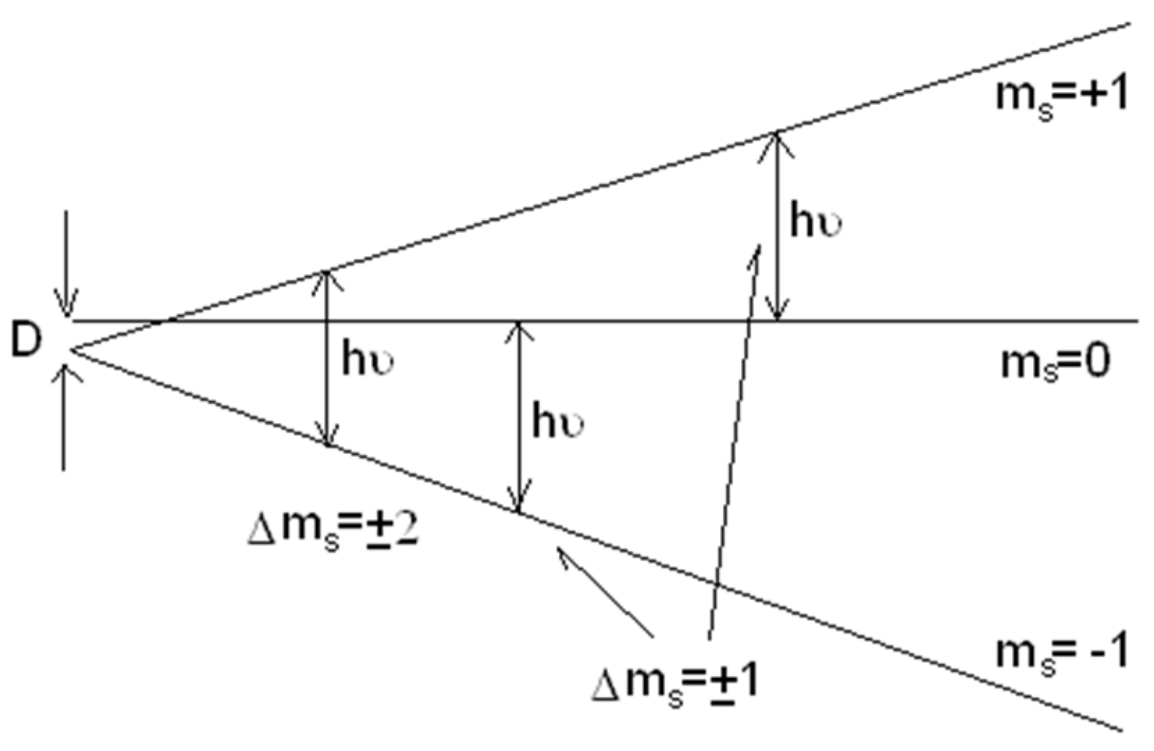

Figure 2.5 Schematic representation of TE sublevel splitting. X-axis is magnetic field. According to equation 2.1, for TEs, resonance can happen either at $\Delta m_{S}= \pm 1$ or 
$\Delta m_{S}= \pm 2$. For $\Delta m_{S}= \pm 2$, only one value of $H$ fulfills the resonance condition. For $\Delta m_{S}= \pm 1$, due to zero field splitting, the resonance happens in a range of $H$, as shown in the figure.

According to Swanson et al. [10] TE powder pattern typically result from the spin Hamitonian

$$
H=\beta \vec{H} \bullet g \bullet \vec{S}+D\left(S_{z}^{2}-\frac{S^{2}}{3}\right)+E\left(S_{x}^{2}-S_{y}^{2}\right)
$$

where $D$ and $E$ are the zero field splitting parameters (Fig. 2.5). Fig. 2.6 shows simulations of the effects of varying $D$ and $E$ values.

To summarize the simulations, the solutions for this Hamiltonian yield the following characteristics of the full field triplet powder pattern [10, 11]:

(1) Steps at $H_{1,2} \approx(h v \pm D) / g \mu_{B}$

(2) Shoulders at $H_{3,4} \approx(h v \pm(D+3 E) / 2) / g \mu_{B}$

(3) Sigularities at $H_{5,6} \approx(h v \pm(D-3 E) / 2) / g \mu_{B}$

Thus, from the powder pattern, one can extract the values of $D$ and $E$. $D$ can be used to estimate the upper bound $r_{U B}$ on the spatial extent of the TE:

$$
r_{U B} \approx\left(\frac{3}{4} g \mu_{B}\right)^{1 / 3}\left(\frac{g \mu_{B}}{D}\right)^{1 / 3} \approx 24.1\left(\frac{g \mu_{B}}{D}\right)^{1 / 3} \AA
$$

And $E / D$ represents the axial symmetry of the TE wavefunctions. ( $E=0$ for axially symmetric and $E=D / 3$ for maximum deviation from axial symmetry).

It should be noted that although this simulation was done to analyze PLDMR results, it is not limited to PLDMR. On the other hand, this simulation is unrelated to 

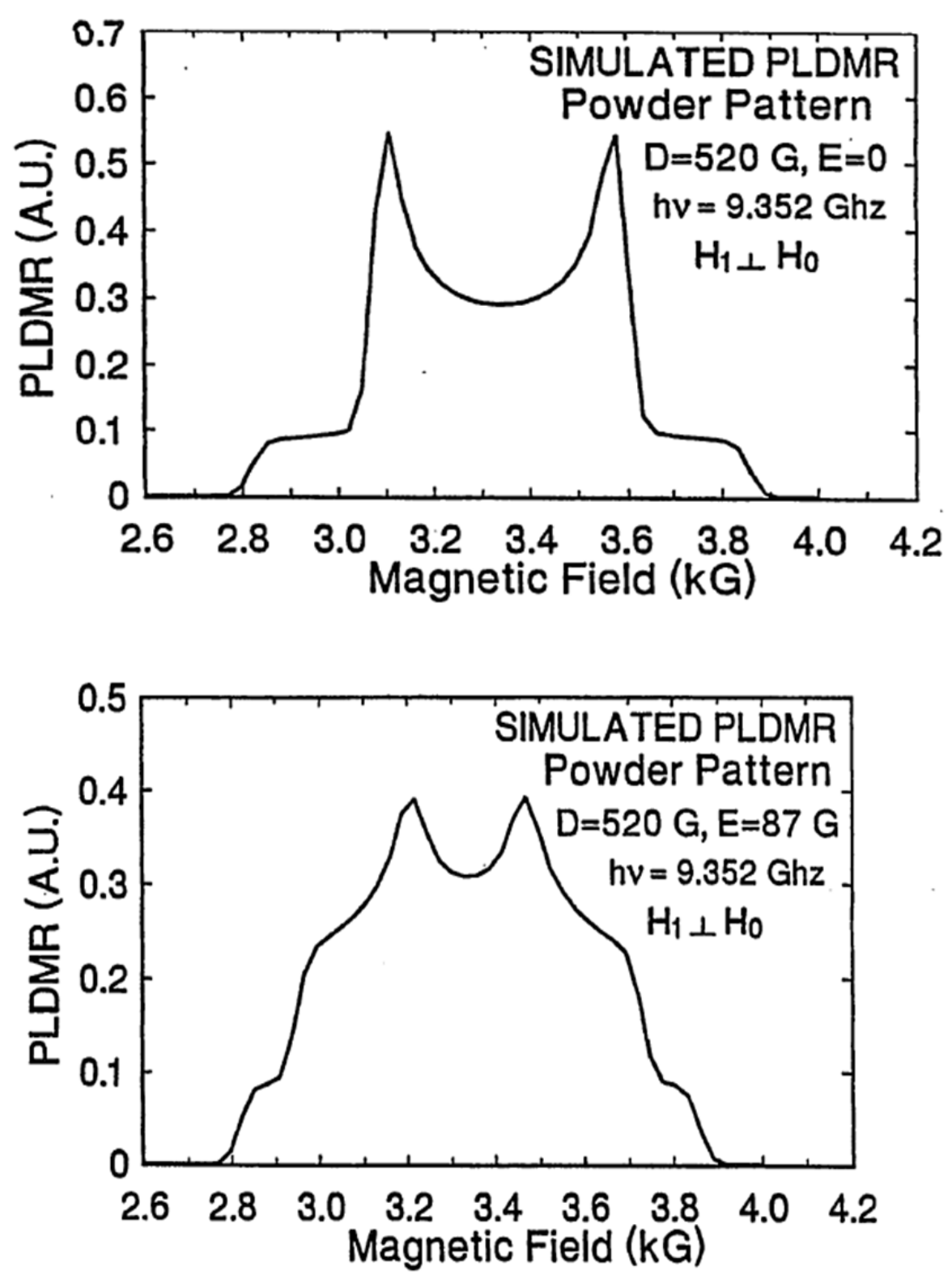

Figure 2.6 Simulated triplet powder pattern with $\Delta m_{S}= \pm 1[10]$

the mechanism responsible for the resonance, leaving the related process unexplained.

The mechanism for the both the broad positive spin-1 powder pattern and the half-field resonance (see below) observed in films and solutions of poly(3-hexylthiophene) (P3HT) and poly(3-dodecylthiophene) (P3DT) [12] remained 
unclear until List et al compared the PLDMR of several polymer blends and proposed the model of reduced SE quenching by TEs that results from a reduced TE population [13].

\subsection{EL detected magnetic resonance (ELDMR)}

ELDMR provides a method to study the spin-related exciton dynamics in OLEDs under real operational conditions. The experimental setup is shown in Figure 2.7.

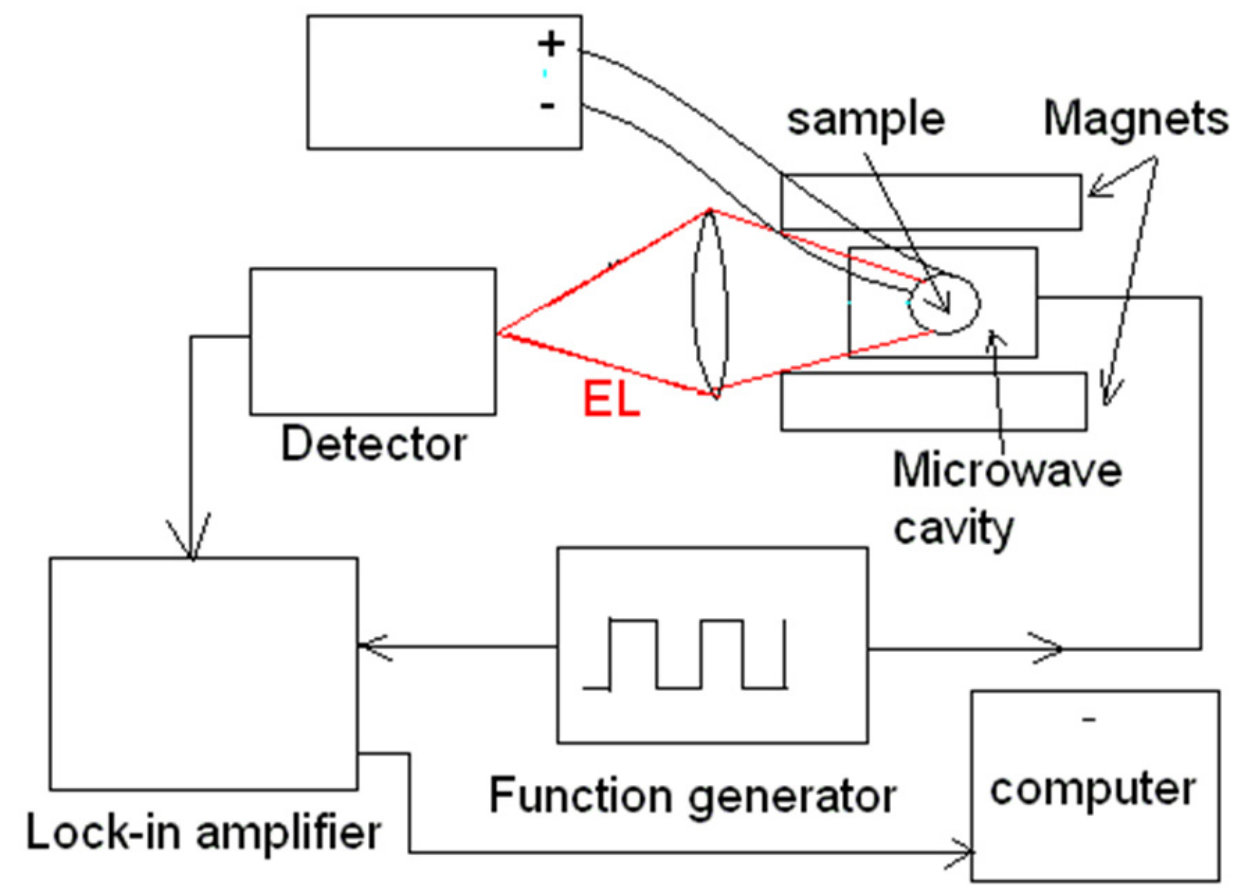

Figure 2.7 Schematic representation of the ELDMR system

Compared with the setup for PLDMR, the only change is that photoexcitation in PLDMR is replaced by electrical current injection. Since the overall process of electroluminescence can be divided into several consecutive steps: carrier injection, carrier transport, recombination and radiative decay, ELDMR can be used to study any spin-related process in these steps. A negative spin-1/2 ELDMR is observed in small molecular OLEDs and polymer LEDs.[14-16] The mechanism for this 
resonance is ascribed to enhanced formation of bipolarons. By comparing the amplitude of this resonance in devices with different buffer layers (between $\mathrm{Al}$ cathode and electron transport layer), $\mathrm{Li}$ et al suggested that the sites for this bipolaron formation locate either near or at the buffer layer.

\subsection{Photocurrent detected magnetic resonance (PCDMR)}

In OSC applications, the devices are photoexcited to generate photocurrent. Monitoring resonance through changes in the photocurrent thus becomes attractive for its potential to provide insights into photovoltaic processes. The schematic experimental setup for PCDMR is shown in figure 2.8.

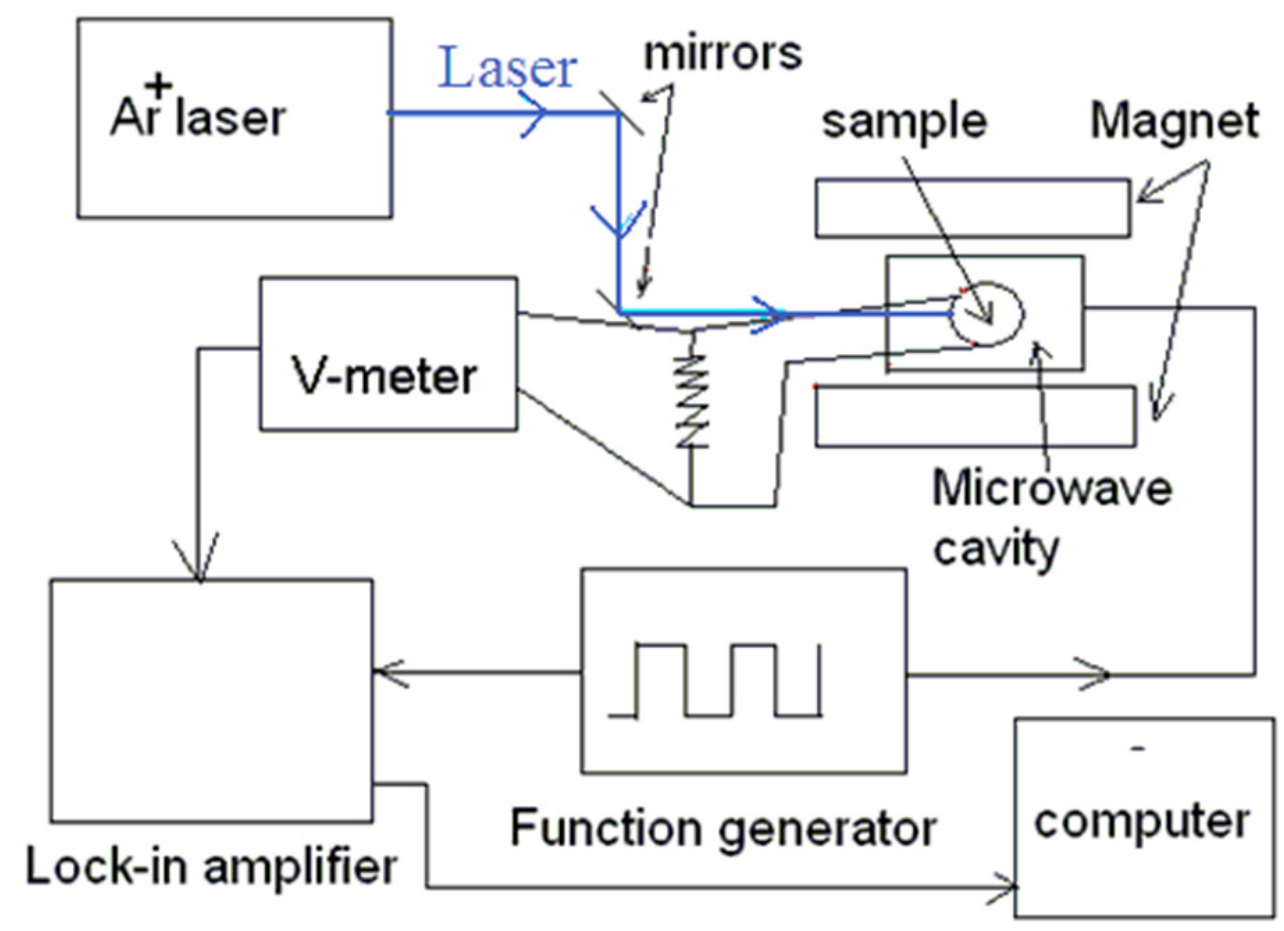

Figure 2.8 Schematic representation of the PCDMR system

As shown, the photocurrent is measured by measuring the voltage drop across a 
standard resistor which is driven by the sample cell. A similar setup is also used for electric current detected magnetic resonance (EDMR) if the photoexcitation source is replaced by a voltage source in series with the standard resistor.

\subsection{Photoinduced absorption detected magnetic resonance (PADMR)}

Besides luminescence and current, the absorption and photoinduced absorption of the material can also be changed under resonance conditions. In this technique, photoexcitation leads to the production of SEs, TEs, and polarons in the material. Since different species have different absorption bands, it is possible to differentiate the contributions of different origins. Photoinduced absorption (PA)-detected magnetic resonance (PADMR) has been studied in methyl-bridged ladder-type poly(p-phenylnenes) (m-LPPPs) and poly(p-phenylene vinylenes) (PPVs) [17, 18]. Negative changes in polaron and TE photoinduced absorption bands were observed in spin-1/2 PADMR. This unambiguously demonstrates that spin-1/2 resonance conditions decrease the polaron and TE populations and thus ruled out the TE-TE annihilation to SEs as a possible mechanism of the spin-1/2 resonance.

\section{References}

[1]. M. Scharnoff, J. Chem. Phys., (1967), 46, 3263

[2]. Charles P. Poole, Jr., Electron Spin Resonance, second edition, (1983)

[3]. J. Kohler et al., C. R. Acad. Sci. Paris, (1997), 324, 373

[4]. M. K. Lee et al., Phys. Rev. Lett., (2005), 94, 137403

[5]. M. Segal et al., Phys. Rev. B, (2005), 71, 245201

[6]. M. Wohlgenannt et al., Phys. Rev. Lett., (2002), 88, 197401 
[7]. C. G. Yang et al., Phys. Rev. Lett., (2007), 99, 157401

[8]. C. G. Yang et al., Phys. Rev. B, (2007), 75, 246201

[9]. C. G. Yang et al., Phys. Rev. Lett., (2006), 96, 089701

[10].L. S. Swanson, Ph. D. Thesis, (1991)

[11].E. J. W. List et al., Phys. Rev. B, (2000), 61, 10807

[12].L. S. Swanson et al., Phys. Rev. Lett., (1990), 65, 1140

[13]. E. J. W. List et al., Phys. Rev. B, (2002), 66, 235203

[14].G. Li et al., Phys. Rev. B, (2004), 69, 165311

[15].G. Li et al., Phys. Rev. B, (2005), 71, 235211

[16].N. C. Greenham et al., Phys. Rev. B, (1996), 53, 13528

[17]. E. J. W. List et al., Phys. Rev. B, (2000), 61, 10807

[18]. X. Wei et al., Phys. Rev. Lett., (1992), 68, 666 


\title{
Chapter 3. ELDMR studies in $\mathrm{Alq}_{3}$ and rubrene-doped $\mathrm{Alq}_{3}$ OLEDs
}

\begin{abstract}
ELDMR is a powerful tool for investigating device physics of OLEDs under real operational conditions. It is sensitive to trapping of various excitons, and thus is very useful for gathering information about trap-related processes. The degradation process and behavior under various current injection levels are investigated using this technique.
\end{abstract}

\subsection{Degradation measured by ELDMR in $\mathrm{Alq}_{3}$ OLEDs}

\subsubsection{Introduction}

OLED applications require long lifetimes. For example, in case of displays, it is generally assumed that a half-life of at least 10,000 hours, at initial display brightness (luminescence) of $100 \mathrm{Cd} / \mathrm{m}^{2}$, is needed [1]. It is due to this requirement that the degradation mechanism of OLEDs has been studied intensly. Degradation generally appears in the form of a decrease in device luminescence. It can proceed through three modes: (i) dark spot degradation, (ii) catastrophic failure, and (iii) intrinsic degradation. The first mode occurs through the formation of non-emissive regions (dark spots) on the device and leads to the decrease in luminescence. It is associated primarily with degradation at device electrodes [2-4]. The second mode occurs through the development of electrical shorts and leads to a sudden decrease or total loss in device luminescence as a result of large leakage current [5]. The third degradation mode is a long-term 'intrinsic' decrease in luminescence of the emissive area. It mainly occurs during device operation [3]. While the first two modes of 
degradation can be effectively controlled by means of proper device encapsulation and adequate control over device fabrication conditions, the intrinsic degradation mode has been far more challenging and continues to be an issue for OLED commercialization.

The EL decreases in intrinsic degradation even when the device is driven by constant current source. This essentially reflects the intrinsic decrease in the EL quantum efficiency. A number of models have been proposed to explain the intrinsic degradation behavior, for example, the morphological instability model in which morphological instability caused by crystallization is believed responsible for degradation [6], the indium migration model in which the degradation is attributed to penetration of indium from the ITO anode into the organic layers, leading to EL quenching [7], and instability of cationic $\mathrm{Alq}_{3}$ model in which the degradation is attributed to the instability of $\mathrm{Alq}_{3}{ }^{+}$that produces EL-quenching byproducts [8]. The last model is, of course, limited to $\mathrm{Alq}_{3}$-based devices. However, since $\mathrm{Alq}_{3}$ is still one of the most widely used small-molecule emitters, and it was reported that Alq ${ }_{3}$-based devices exhibit interesting organic magneto resistance (OMR) effects $[9,10]$, it is important to understand the degradation mechanism in such devices.

In this study, we observed the degradation behavior in $\mathrm{Alq}_{3}$-based devices through room temperature ELDMR measurements. The development of the resonance signal is recorded and the mechanism behind the phenomenon is discussed.

\subsubsection{Experimental}

Sublimed

$\mathrm{Alq}_{3}$

and

hole-transporting 
$\mathrm{N}, \mathrm{N}^{\prime}$-di(naphthalene-1-yl)-N,N'-diphenyl-benzidine (NPD) were purchased from $\mathrm{H}$. W. Sands. CsF was purchased from Sigma-Aldrich. All materials were used as received.

All devices were fabricated on the glass/ITO substrates in a thermal evaporation chamber within a glovebox with a base pressure of $\sim 2 \times 10^{-6}$ mbar. The cleaned ITO substrates were dried by blowing nitrogen and then treated in a UV ozone oven to increase the work function of the ITO and hence facilitate hole injection, as described elsewhere [11]. $5 \mathrm{~nm}$ of CuPC, $50 \mathrm{~nm}$ of NPD, $40 \mathrm{~nm}$ of $\mathrm{Alq}_{3}, 1 \mathrm{~nm}$ of CsF and 100 $\mathrm{nm}$ of Al were deposited sequentially.

For ELDMR measurements, the fabricated devices were placed in the quartz dewar of an Oxford Instruments cryostat inside an optically accessible $9.35 \mathrm{GHz}$ $\mathrm{X}$-band microwave cavity. The changes in the EL induced by the X-band microwaves at the field for resonance were detected by feeding the signal into a lock-in amplifier referenced by the microwave chopping frequency $f_{\mu}=500 \mathrm{~Hz}$. During the room-temperature experiments, the device was protected by flowing high purity dry nitrogen through the cryostat.

\subsubsection{Results and discussion}

A negative spin-1/2 ELDMR resonance can be observed at room temperature when the sample is driven at $U=18 \mathrm{~V}$, which generated a current density $j=0.25$ $\mathrm{mA} / \mathrm{mm}^{2}$ through the device. The g-factor calculated from the peak position is 2.0025 . The full width at half maximum (FWHM) is $\sim 20$ Gauss. This is consistent with

previous studies on similar devices [12]. The microwave power when it is not 
chopped is $160 \mathrm{~mW}$. In the measurements, the microwave power was chopped at $500 \mathrm{~Hz}$.

Figure 3.1 shows the development of the negative spin-1/2 resonance through multiple runs. The amplitude of this resonance monotonically increases with increasing time. The line shape of the resonance is stable.

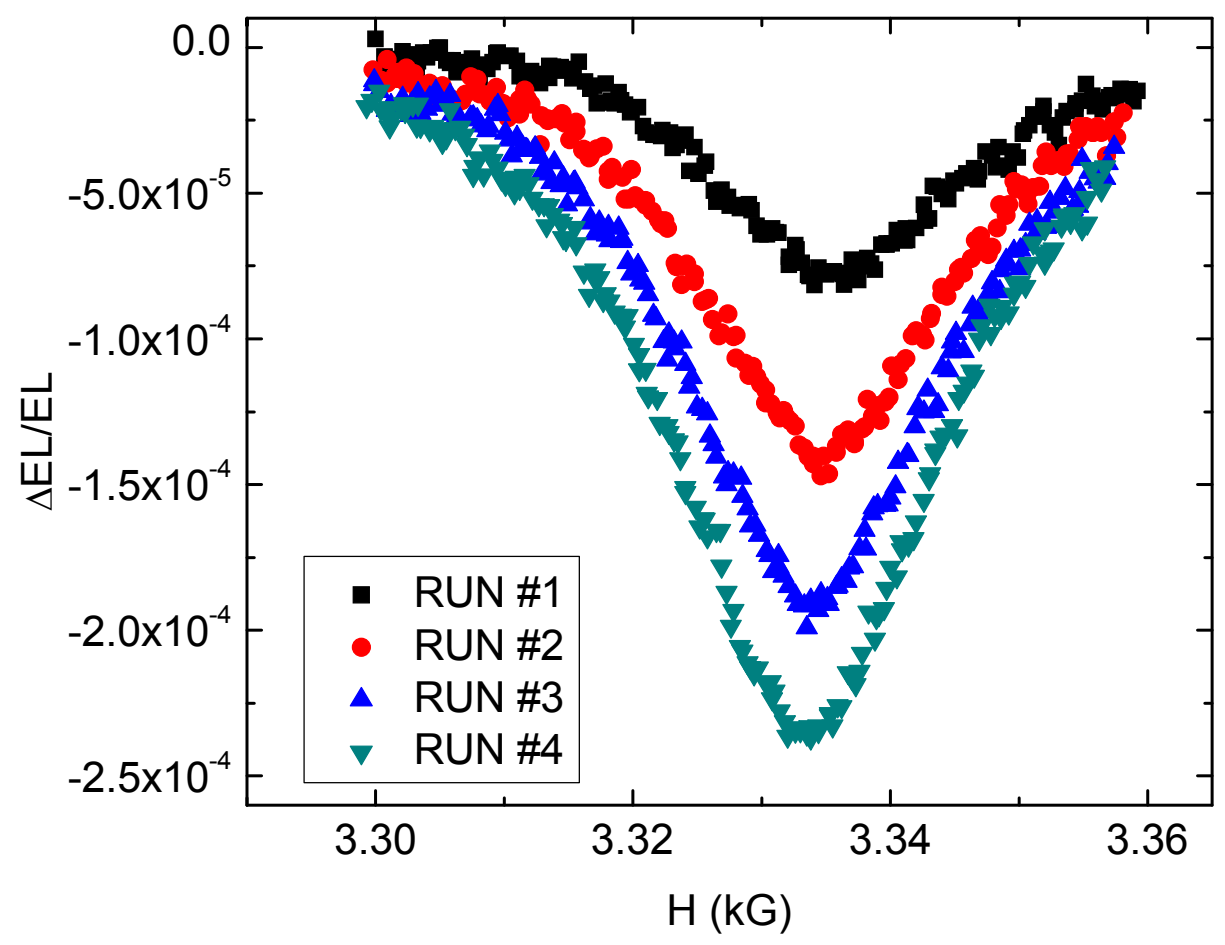

Figure 3.1 Evolution of the negative spin-1/2 ELDMR in ITO/Alq $3 / \mathrm{LiF} / \mathrm{Al}$ OLEDs right after the device fabrication. The time interval between each run is 10 minutes.

$$
\mu \text {-wave power } P_{\mu}=160 \mathrm{~mW} \text {, bias } U=18 \mathrm{~V}, \mathrm{~T}=293 \mathrm{~K}
$$

Figure 3.2 shows the amplitude of the resonance $\Delta I_{E L} / I_{E L}$ vs. time of a similar device. Note the resonance is negative, so the amplitude increases by $12 \%$ in $250 \mathrm{~min}$. It should be pointed out that the device degrades much faster when it is initially 
driven by the current source, so the degradation effect shown in Fig 3.2 is much less than that in Fig 3.1. In the experiment corresponding to Figure 3.2, it is believed that some degradation had already taken place when the system was adjusted for testing. However, since we are more concerned about the long-term 'intrinsic' degradation, as mentioned previously, it is reasonable to monitor the slower degradation.

As one can see, a decrease in the total EL through other mechanisms such as growing dark spots could lead to a "false" increase in $\Delta I_{E L} / I_{E L}$. To exclude this effect, $\Delta I_{E L}$ as a function of time is plotted in Figure 3.3. As shown in the figure, $|\Delta E L|$ increases by $\sim 9 \%$ in $250 \mathrm{~min}$. Thus, the degradation observed in this experiment is mainly manifest in the resonance getting stronger with time.

It is generally believed that the negative spin-1/2 ELDMR in $\mathrm{Alq}_{3}$ devices is due to enhanced formation of spinless bipolarons under resonance conditions [12]. According to their EDMR results, Gang Li et al. suggested that the EL decreases simply due to decrease in $j$ under resonance conditions, since bipolarons have lower mobility. However, instead of driven by voltage source, the sample was driven at constant current in our experiments. Thus the scenario proposed can not explain the phenomenon observed here. The negative spin-1/2 ELDMR under constant current suggests that compared with polarons, bipolarons are more effective quenching centers for singlet excitons (SEs). Under resonance conditions, more SEs are dissociated by more bipolarons, which in turn leads to more quenching of EL. 


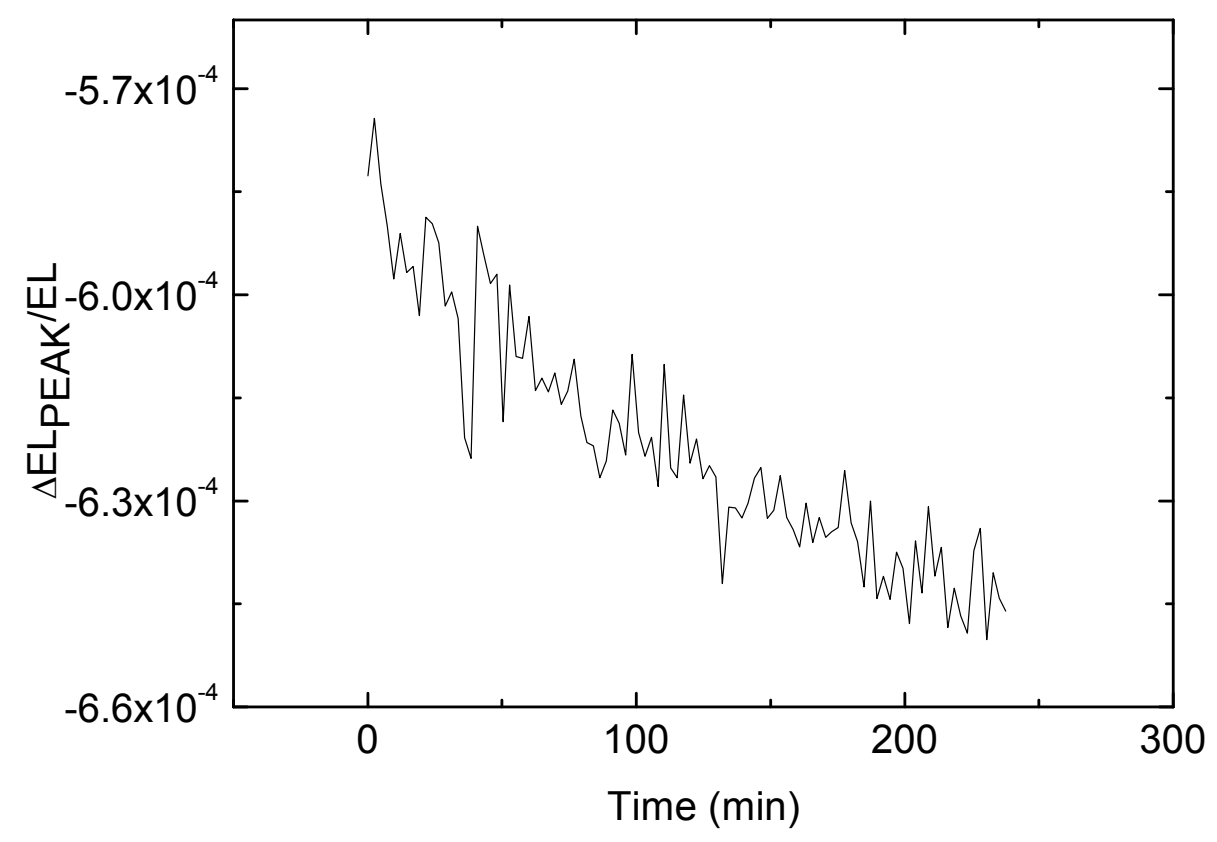

Figure 3.2 ELDMR vs time in an $\mathrm{Alq}_{3}$ OLED. $\mu$-wave power $P_{\mu}=160 \mathrm{~mW}, j=6$ $\mathrm{mA} / \mathrm{cm}^{2} . T=293 \mathrm{~K}$

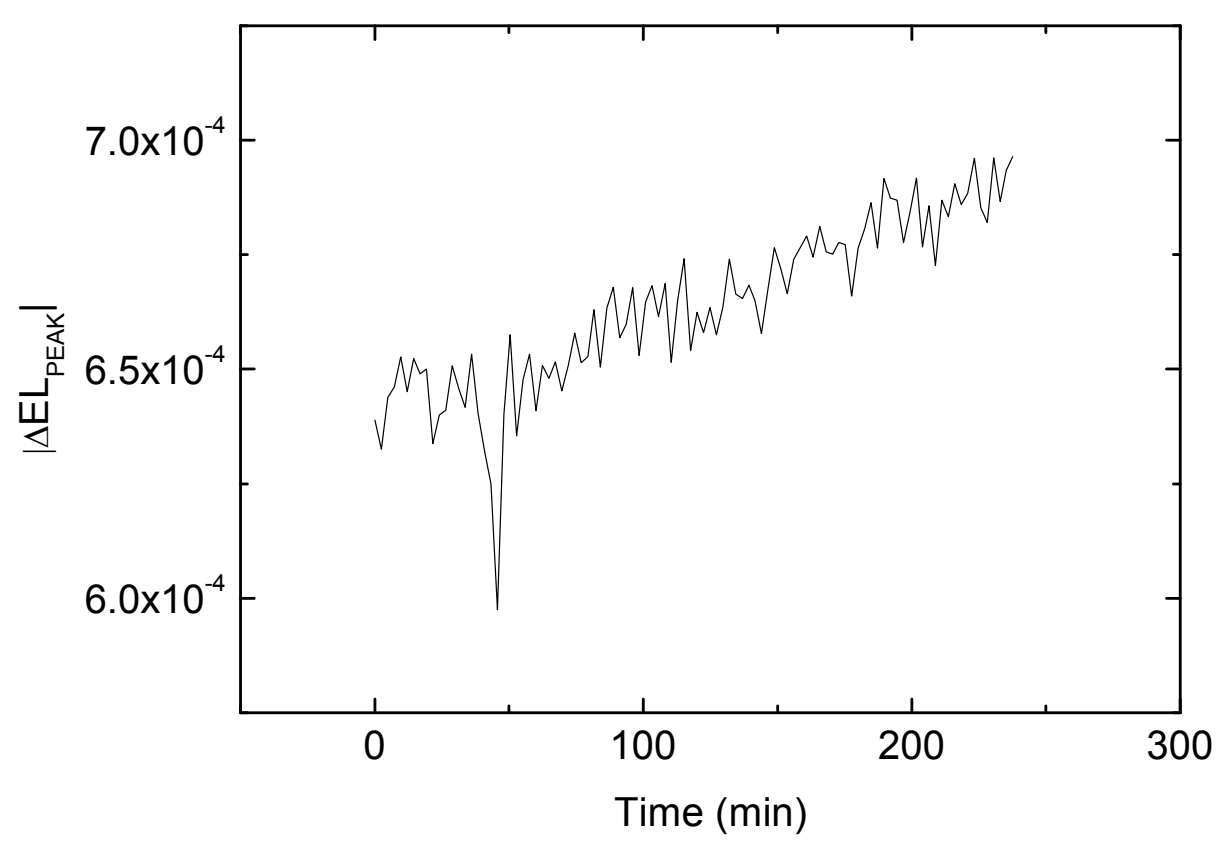

Figure 3.3 Resonance induced change $|\Delta E L|$ vs time. $\mu$-wave power $P_{\mu}=160 \mathrm{~mW}$,

$$
j=6 \mathrm{~mA} / \mathrm{cm}^{2} . T=293 \mathrm{~K}
$$


The increase in $|\Delta E L|$ with increasing time indicates more bipolarons are formed after degradation. The most simplified rate equation to depict this phenomenon can be written as follows:

$\frac{d b p}{d t}=G_{b p}-\frac{b p}{\tau}$

where $b p$ is the population of spin-0 bipolarons, $G_{b p}$ is the generation rate of these bipolarons and $\tau$ is the effective lifetime of bipolarons, including all first order decay channels. Obviously, at steady state

$b p=G_{b p} \times \tau$

Under resonance conditions, like-charged polaron pairs in a non-zero spin configuration convert to spin- 0 configuration with the help of magnetic resonance, leading to a higher generation rate of bipolarons $G_{b p}{ }^{O N}$. Assuming $\tau$ does not change significantly under resonance conditions, $|\Delta E L| \propto \Delta b p=\left(G_{b p}^{o N}-G_{b p}\right) \times \tau=\left(\frac{G_{b p}{ }^{o N}}{G_{b p}}-1\right) \times b p$

Since $\frac{G_{b p}{ }^{O N}}{G_{b p}}$ should only depend on resonance conditions such as microwave power, with same resonance conditions, a increase in $|\Delta E L|$ most probably indicates a higher $b p$, i.e., the quenching of SEs by bipolarons becomes more significant upon degradation. Indeed, it is proposed that due to the continuous formation of traps or increased disorder induced by electrical field-induced stress, more bipolarons are generated in the device [13]. This scenario has also been invoked to explain the increased OMR in $\mathrm{Alq}_{3}$-based devices upon degradation. Our observation here is in good agreement with the proposed scenario. 
To verify the validation of the simple analysis above, i.e., the model with single constant effective bipolaron lifetime, $\Delta I_{E L} / I_{E L}$ vs $f_{M}$ was measured and the model was used to fit the experiment data, as shown in Figure 3.4.

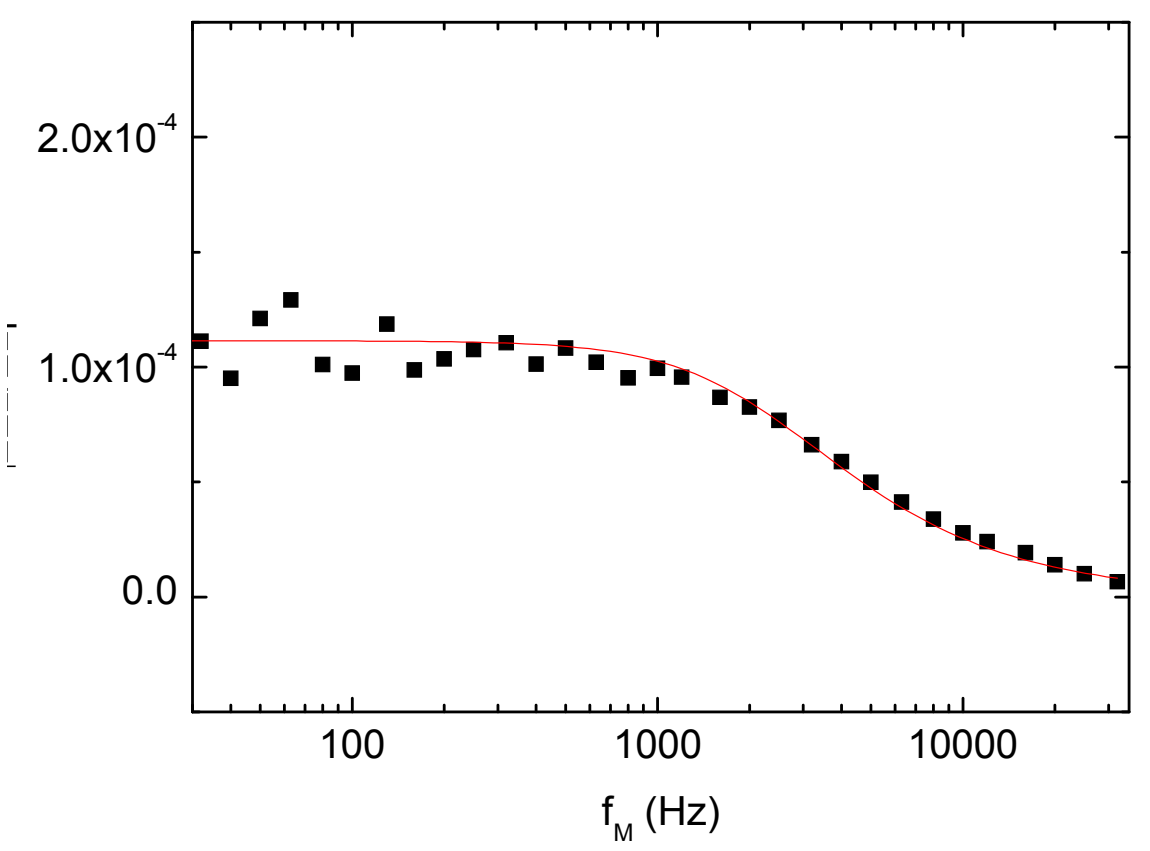

Figure 3.4 Magnitude of the spin-1/2 ELDMR for Alq3-based OLEDs vs $f_{M}$. The solid line is the fit according to the model presented in the text.

For frequency dependence, we define $X^{m}$ as the Fourier coefficient for a given modulated rate or species, $X(t)$, so that

$$
X(t)=\operatorname{Re}\left\{\sum_{m} X^{m} e^{i m \omega_{m} t}\right\}
$$

where $m$ refers to harmonics of microwave chopping frequency $\omega_{m}$.

Thus, from the rate equation,

$$
|\Delta E L| \propto b p^{1}=\operatorname{Re}\left\{\frac{G^{1}}{i \omega+\frac{1}{\tau}}\right\}=\frac{G^{1}}{\sqrt{\omega^{2}+\frac{1}{\tau^{2}}}}
$$


The fitting is shown in Figure 3.4 as red line. As one can see, with $\tau=68 \mu$ s there is good agreement with the measured behavior.

\subsubsection{Conclusion}

To conclude, ELDMR measurements on $\mathrm{Alq}_{3}$-based OLEDs indicate enhanced bipolaron formation after degradation. This is consistent with other experiments such as OMR. Thus, we conclude that intrinsic degradation in $\mathrm{Alq}_{3}$-based devices generates traps that induce bipolarons.

\subsection{ELDMR in rubrene-doped $\mathrm{Alq}_{3}$-based devices}

\subsubsection{Introduction}

Doping of organic light-emitting layers with fluorescent or phosphorescent dyes has been widely used to enhance the electroluminescence (EL) efficiency or to tune the emission color since the first demonstration of efficient EL in OLEDs [14-18]. Doping can also enhance the device stability by facilitating radiative recombination of injected electrons and holes and thereby reducing unstable excited states $[8,14,19]$. Rubrene is well known for its use as a yellow light source in light sticks. It is also widely used as a dopant in OLEDs, especially for white light emission applications $[20,21]$. It is reported that doping rubrene into $\mathrm{Alq}_{3}$ can greatly enhance the device stability, improving the device lifetime 100 fold [22]. In addition, the EL efficiency of red fluorescent dyes doped into $\mathrm{Alq}_{3}$ was reported to be improved by additional doping of rubrene, which assists the energy transfer from $\mathrm{Alq}_{3}$ to the red dopants [23, 24]. 
In this study, we investigated the ELDMR of OLEDs with rubrene doped into $\mathrm{Alq}_{3}$ as the active layer. The behavior of the resonance is different from Alq $\mathrm{A}_{3}$-only devices. The mechanism driving the ELDMR is discussed.

\subsubsection{Experimental}

Sublimed $\mathrm{Alq}_{3}$ and hole transporting material NPD were purchased from $\mathrm{H}$. W. Sands. Rubrene and CsF were purchased from Sigma-Aldrich.

All devices were fabricated through the same procedures as described in Sec. 3.1. $5 \mathrm{~nm}$ of CuPC, $50 \mathrm{~nm}$ of NPD, $10 \mathrm{~nm}$ of rubrene-doped $\mathrm{Alq}_{3}, 30 \mathrm{~nm}$ of $\mathrm{Alq}_{3}, 1 \mathrm{~nm}$ of $\mathrm{CsF}$, and $100 \mathrm{~nm}$ of $\mathrm{Al}$ were deposited sequentially. The doping ratio was $2 \mathrm{wt} \%$ rubrene in $\mathrm{Alq}_{3}$, unless otherwise mentioned.

ELDMR measurements were carried out in the same way as described in the previous section and Chapter 2. During the experiments, the device was protected by flowing high purity dry nitrogen through the cryostat.

\subsubsection{Results and Discussions}

Spin-1/2 ELDMR

Figure 3.5 shows the Spin-1/2 ELDMR in a rubrene-doped $\mathrm{Alq}_{3}$ device at various current densities $j$. As seen, at $j<10 \mathrm{~mA} / \mathrm{cm}^{2}$, a negative resonance with g-factor $g=2.0023$ and FWHM $\sim 15 \mathrm{G}$ can be seen. $\left|\Delta I_{E L} / I_{E L}\right|$ of this negative resonance decreases with increasing $j$. When the current increases even higher, a positive resonance appears with a similar $g$ factor but slightly broader lineshape and overlapping the negative resonance. $\Delta I_{E L} / I_{E L}$ of this positive resonance increases with $j$. Further increasing $j$ to $>80 \mathrm{~mA} / \mathrm{cm}^{2}$ renders the negative resonance unobservable. 


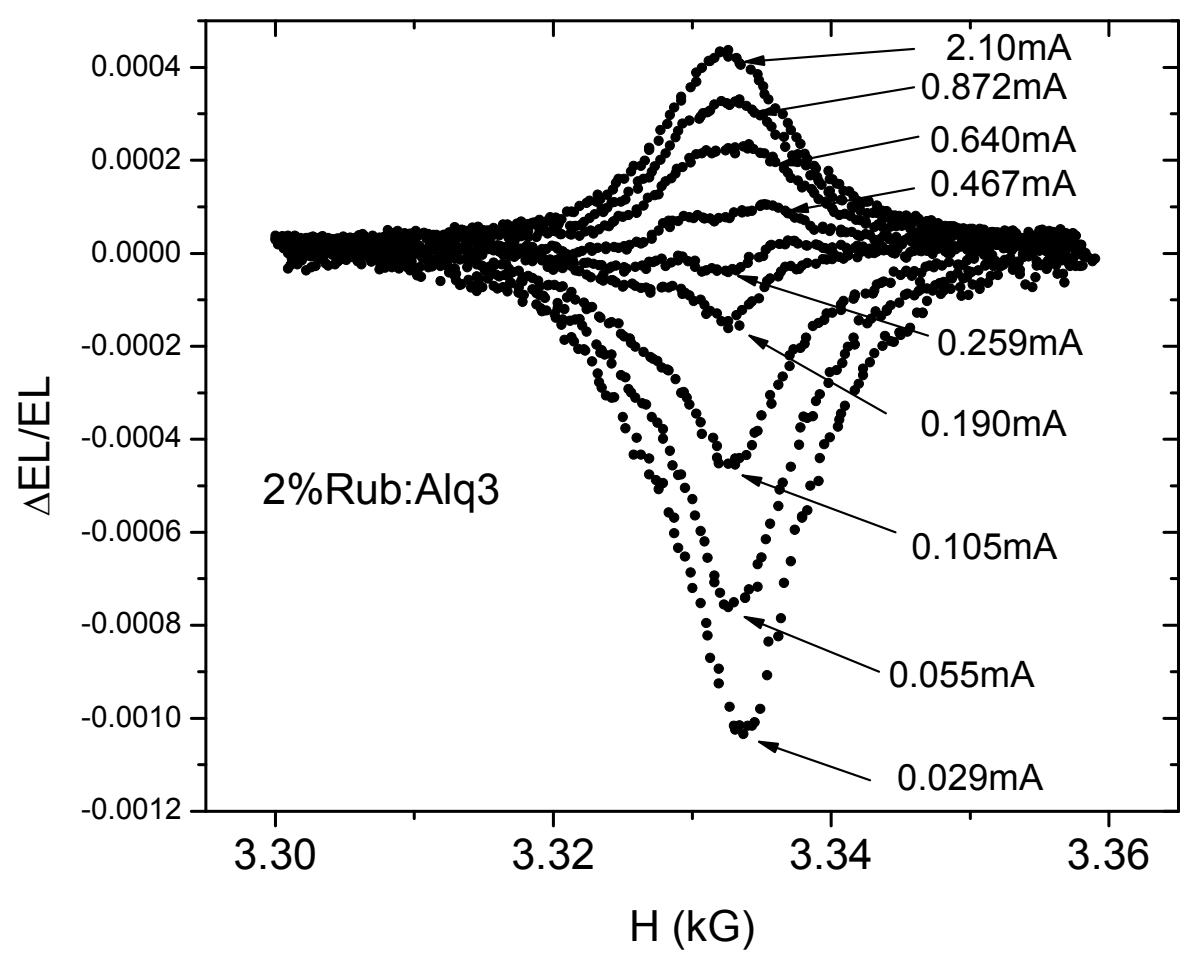

Figure 3.5 Spin-1/2 ELDMR in a Rubrene doped Alq3 OLED at various current injection levels. $\mu$-wave power $P_{\mu}=160 \mathrm{~mW}, T=293 \mathrm{~K}$. Active area $\mathrm{A}=4 \mathrm{~mm}^{2}$.

The negative resonance is similar to the spin- $1 / 2$ negative ELDMR in pure $\mathrm{Alq}_{3}$ devices. As mentioned in Sec. 3.1, it is assigned to the enhanced formation of bipolarons under resonance conditions. However, in the rubrene-doped $\mathrm{Alq}_{3}$ devices, the lineshape is narrower. This can be explained by the trapping effect of rubrene. It has been well established that rubrene traps charge and is a direct recombination center in these devices [25]. On the other hand, bipolarons are stabilized by Coulombically bounding to oppositely charged defects or impurities, to form trions [26]. Considering the dipolar broadening contribution to the resonance lineshape, smaller FWHM implies shorter average distance $d$ between these counterions [12]. In 
our case, doping of rubrene leads to higher trap density in the device, i.e., smaller d, thus leads to a narrower negative spin-1/2 ELDMR lineshape.

The positive spin-1/2 resonance has not been previously observed in $\mathrm{Alq}_{3}$-only devices. Figure 3.6 shows the spin-1/2 ELDMR in a $\mathrm{Alq}_{3}$-only device at various $j$. Even at very high $j \sim 200 \mathrm{~mA} / \mathrm{cm}^{2}$, when the device was driven at $20 \mathrm{~V}$, only the negative resonance can be seen, even though $\left|\Delta I_{E L} / I_{E L}\right|$ decreased with increasing $j$. This behavior was also reported in previous ELDMR studies of $\mathrm{Alq}_{3}$-only devices [12].

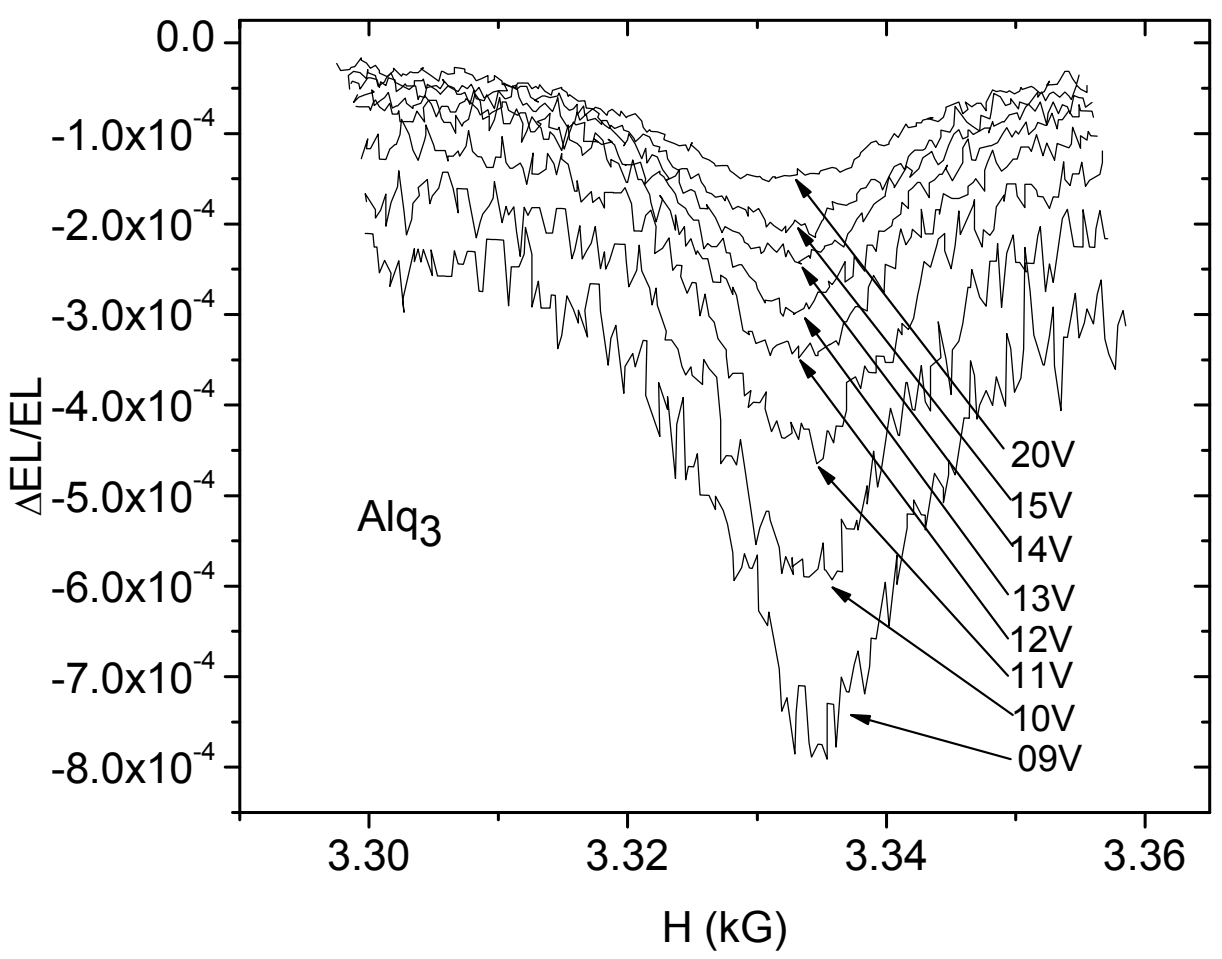

Figure 3.6 Spin-1/2 ELDMR in an Alq3 OLED at various $j$. $\mu$-wave power $P_{\mu}=160$ $m W, T=293 K$. Active area for the device $A=4 \mathrm{~mm}^{2}$

To understand the mechanism behind the positive spin-1/2 resonance, one can compare it with the PLDMR results in MEH-PPV films. It has been reported that even 
at room temperature, a positive spin-1/2 PLDMR resonance can be seen in MEH-PPV films $[27,28]$. The g-factor of the positive PLDMR is similar to the g-factor of the positive ELDMR in rubrene-doped $\mathrm{Alq}_{3}$ devices, consistent with a similar mechanism for both.
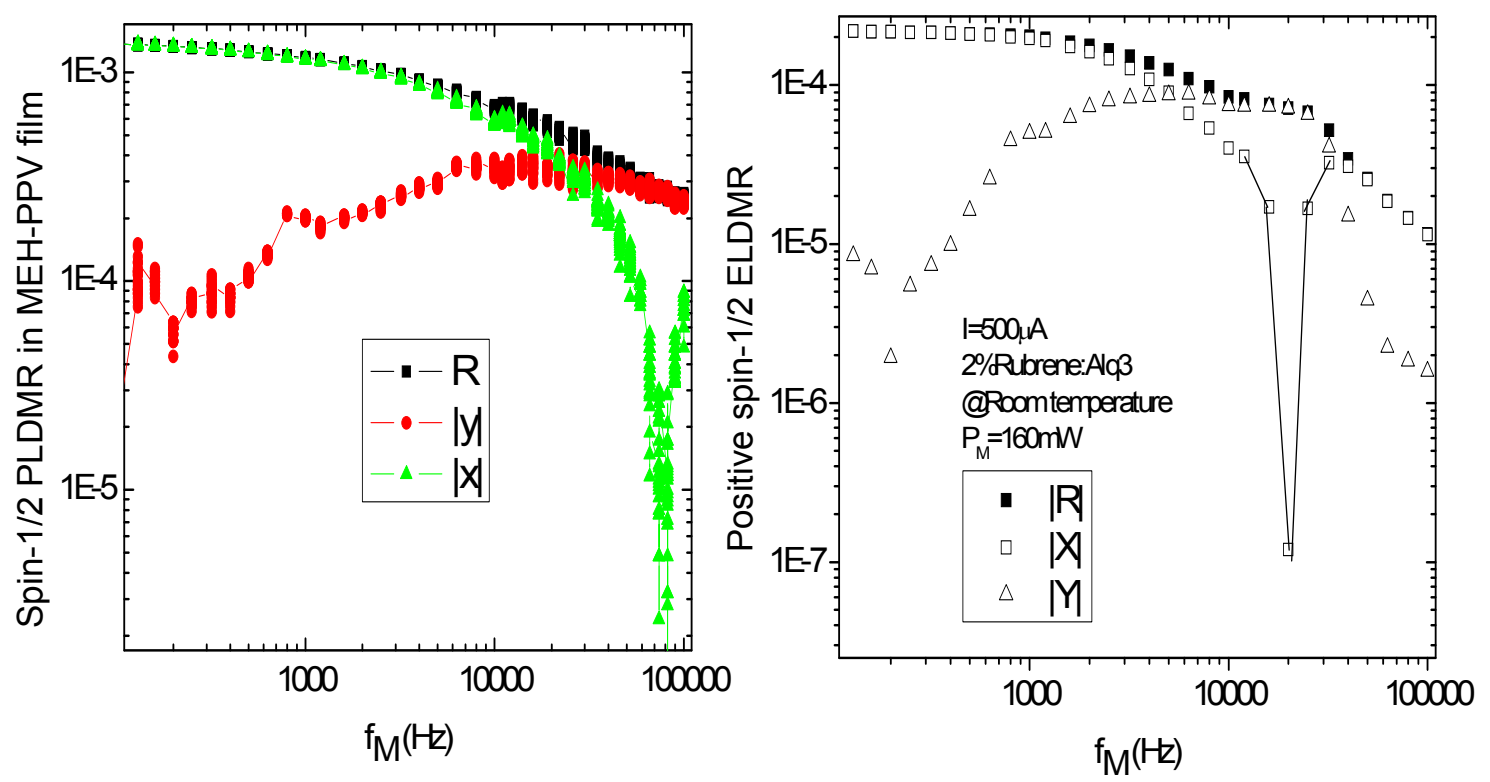

Figure 3.7 Comparison of the $f_{M}$ dependence of the amplitude $R$, in-phase component

$X$, and quadrature component $Y$ between (a) the positive spin-1/2 PLDMR in an MEH-PPV film and (b) the positive spin-1/2 ELDMR in a rubrene doped Alq ${ }_{3} O L E D$.

To further compare the PLDMR of the MEH-PPV film and the ELDMR of the rubrene-doped $\mathrm{Alq}_{3}$ OLED, the microwave chopping frequency dependence of the two single modulated resonances are shown in Figure 3.7. As one can see, the behavior of the resonances is similar, including the in-phase and out-of-phase components. Note that both resonances were detected with the same spectrometer. Since the frequency dependence of ODMR is strongly dependent on the mechanism behind the resonance, this similarity clearly demands a similar mechanism for both. 
The mechanism for the positive PLDMR has been ascribed to mainly two types of models: (i) The spin-dependent recombination (SDR) model assumes that SE formation is favored over TE formation in organic light emitting materials, so that the positive spin 1/2 PLDMR is due to enhanced delayed fluorescence resulting from magnetic resonance-induced net conversion of triplet polaron pairs to singlet polaron pair [29]. (ii) The TE-polaron quenching (TPQ) model assumes that the positive spin 1/2 PLDMR and ELDMR resonance is due to a reduction in the quenching rate of SEs by TEs and polarons, which itself results from a reduction in the TE and polaron populations through enhanced TE-polaron annihilation under resonance conditions $[27,28]$. However, it was shown that the double modulation PLDMR (DM-PLDMR) is consistent with the TPQ model while inconsistent with the SDR model. Hence, to compare the ELDMR in the rubrene-doped $\mathrm{Alq}_{3}$ device with the PLDMR in the MEH-PPV film, a double modulation ELDMR (DM-ELDMR) experiment was also carried out, where both the microwave power and $j$ were modulated. The result is shown in Figure 3.8. As clearly seen, the DM-ELDMR result is similar to the DM-PLDMR, i.e., the amplitude of the double modulated resonance is essentially independent of the bias chopping frequency $f_{V}$. Thus, the mechanism behind the positive spin-1/2 ELDMR in rubrene-doped $\mathrm{Alq}_{3}$ devices is the same as the mechanism behind the positive spin-1/2 PLDMR in MEH-PPV films, i.e., enhanced annihilation of TE-polaron pairs (TPQ) that reduces the TE and polaron populations under resonance conditions, and in turn reduces the quenching of SEs by TEs and polarons. Higher $j$ produces more polarons on $\mathrm{Alq}_{3}$ moledcules, which enhances the 
TPQ effects, including ELDMR.

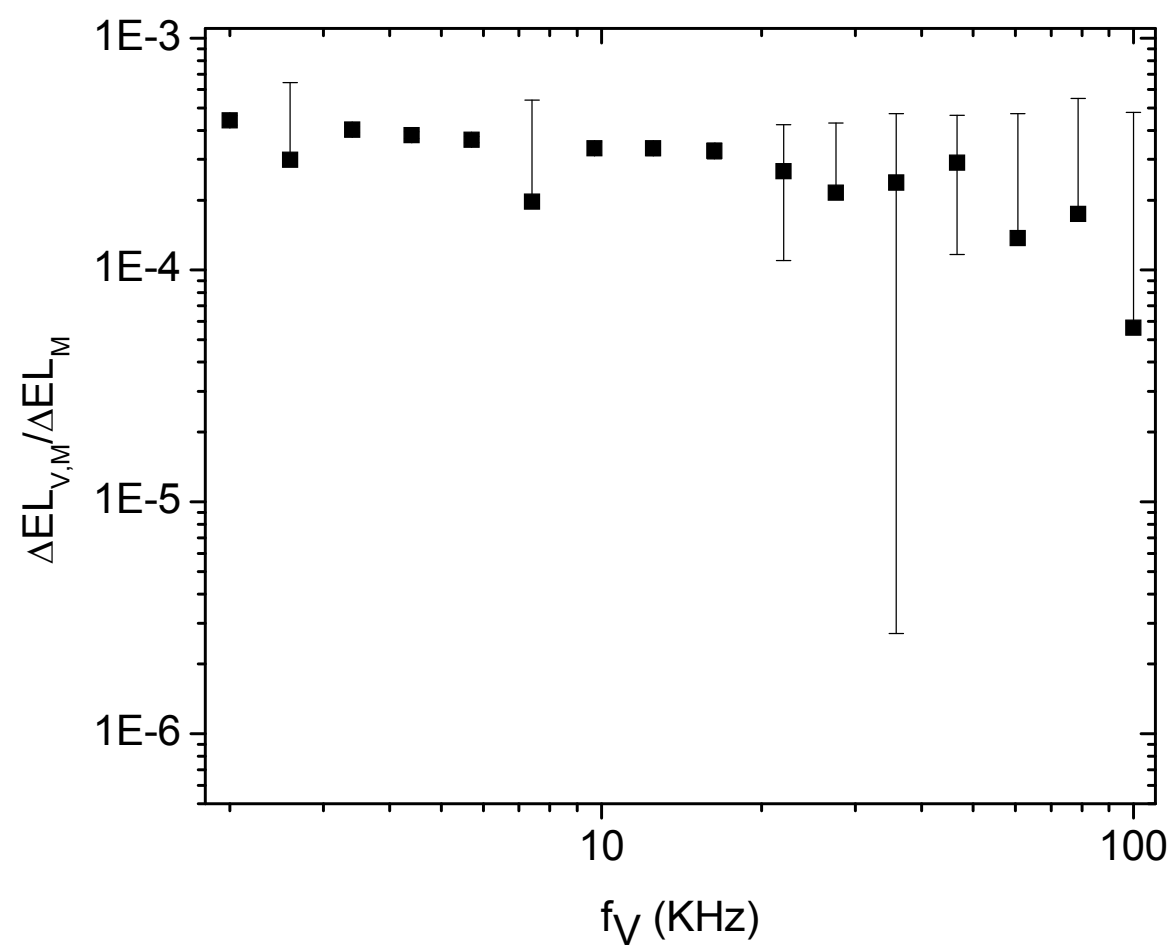

Figure 3.8 Double modulated ELDMR as a function of modulation frequency $f_{V}$.

$$
P_{\mu}=160 \mathrm{~mW}, J=6 \mathrm{~mA} / \mathrm{cm}^{2} . T=293 \mathrm{~K}
$$

With higher current injection, not only the positive spin-1/2 resonance gets stronger, but also a broad triplet powder pattern ELDMR around $g=2$ can be seen. Figure 3.9 shows such a full field triplet powder pattern. A similar triplet powder pattern has been reported in PLDMR results from various polymer films [30-32]. The mechanism for this triplet resonance was investigated by List et al. and ascribed to the reduction of TE-SE annihilation, i.e., the same TPQ model as assigned to the positive spin-1/2 resonance.[33]

Figure 3.10 shows the half-field triplet powder pattern ELDMR which corresponds to $\Delta m_{S}= \pm 2$ transitions among the TE spin sublevels. 


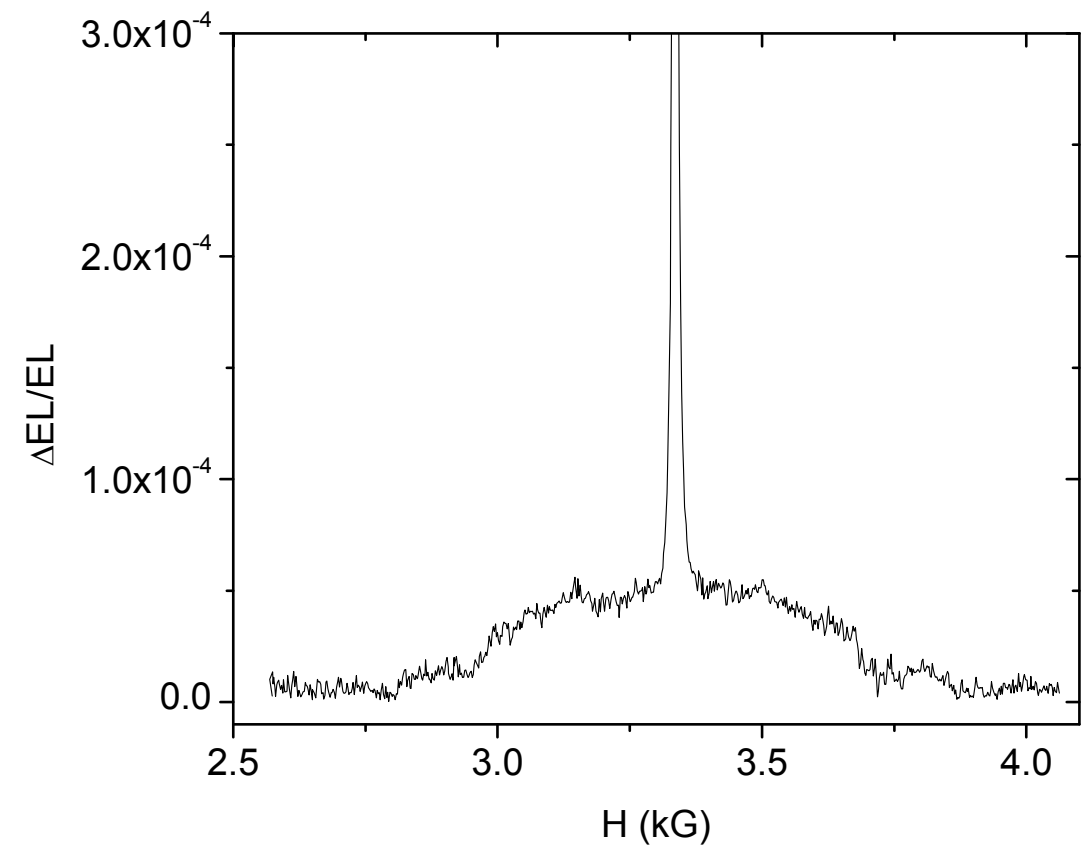

Figure 3.9 Triplet powder pattern ELDMR corresponding to $\Delta m_{S}= \pm 1 . P_{\mu}=160 \mathrm{~mW}$,

$$
J=40 \mathrm{~mA} / \mathrm{cm}^{2} . T=293 \mathrm{~K}
$$

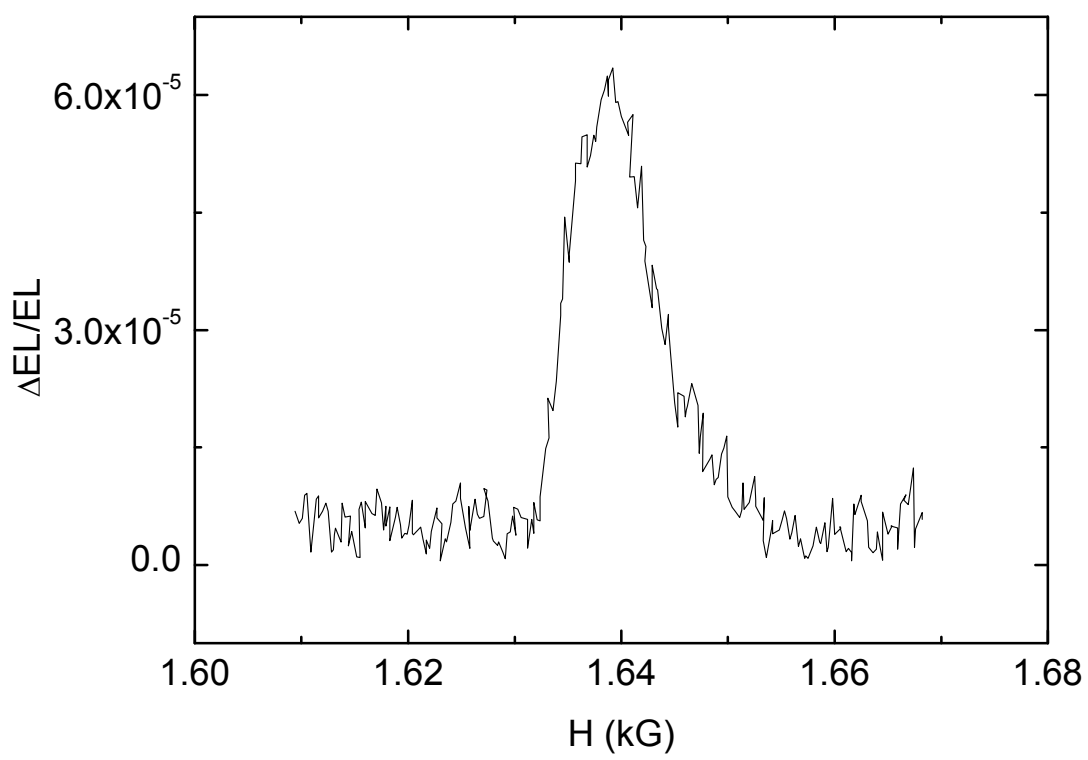

Figure 3.10 Triplet powder pattern ELDMR corresponding to $\Delta m_{S}= \pm 2 . P_{\mu}=160 \mathrm{~mW}$,

$$
J=40 \mathrm{~mA} / \mathrm{cm}^{2} . T=293 \mathrm{~K}
$$

The observation of a triplet powder pattern is due to the anisotropic 
electron-electron spin interaction, which is given by the spin Hamiltonian $H_{s s}=J_{0} \overrightarrow{S_{1}} \bullet \overrightarrow{S_{2}}+D\left(S_{1 z} S_{2 z}-\frac{1}{3} \overrightarrow{S_{1}} \bullet \overrightarrow{S_{2}}\right)+E\left(S_{1 x} S_{2 x}-S_{1 y} S_{2 y}\right)+\vec{d} \bullet \overrightarrow{S_{1}} \times \overrightarrow{S_{2}}$

where $J_{0}$ is the exchange term and $D$ and $E$ are the zero-field-splitting parameters. Within a first approximation $J_{0} \overrightarrow{S_{1}} \bullet \overrightarrow{S_{2}}$ can be omitted since it only contributes to the singlet-triplet splitting and does not affect the transitions in the triplet manifold and $\vec{d} \bullet \overrightarrow{S_{1}} \times \overrightarrow{S_{2}}$ also vanishes within the triplet manifold. So the modified Hamiltonian only contains the second and third terms in Eq. (3.6) [34].

The solutions for this Hamiltonian yield the following characteristics of the full field triplet powder pattern:[35]

(4) Steps at $H_{1,2} \approx(h v \pm D) / g \mu_{B}$

(5) Shoulders at $H_{3,4} \approx(h v \pm(D+3 E) / 2) / g \mu_{B}$

(6) Singularities at $H_{5,6} \approx(h v \pm(D-3 E) / 2) / g \mu_{B}$

Comparing these values with the triplet ELDMR powder pattern shown in figure 3.9, one gets $D / g \mu_{B}$ and $E / g \mu_{B}$ of $520 \mathrm{G}$ and $50 \mathrm{G}$ respectively. As shown by Swanson et al. [31], an upper bound $r_{U B}$ on the spatial extent of the TE can be determined from the following equation for $D$ :

$D=\frac{3}{4}\left(g \mu_{B}\right)^{2}\left\langle\left(r^{2}-3 z^{2}\right) / r^{5}\right\rangle$

where $\left\langle\left(r^{2}-3 z^{2}\right) / r^{5}\right\rangle$ is the expectation value of $\left(r^{2}-3 z^{2}\right) / r^{5}$ in the TE eigenstate.

Since $\left\langle\left(r^{2}-3 z^{2}\right) / r^{5}\right\rangle \leq\left\langle 1 / r^{3}\right\rangle$

$r_{U B} \approx\left(\frac{3}{4} g \mu_{B}\right)^{1 / 3}\left(\frac{g \mu_{B}}{D}\right)^{1 / 3} \approx 24.1\left(\frac{g \mu_{B}}{D}\right)^{1 / 3} \AA$ 
For the value of $D$ obtained above, we get $r_{U B} \approx 2.9 \AA$.

The foregoing values of $r_{U B}$ and $D$, and the triplet pattern shown in Figs. 3.9 and 3.10 are similar to those observed in PLDMR of conjugated polymers. Thus we assign the TE resonance to the same mechanism, i.e., TPQ. It is not surprising that with the same mechanism, both the positive spin-1/2 ELDMR and spin-1 triplet powder pattern ELDMR increase with increasing $j$.

Degradation of the spin-1/2 ELDMR in rubrene-doped $\mathrm{Alq}_{3}$ devices

As mentioned before, ODMR measurements can provide useful information on device degradation. Figure 3.11 shows the evolution of the spin-1/2 ELDMR for a rubrene-doped $\mathrm{Alq}_{3}$ OLED (protected with flowing high purity nitrogen) at constant $j$.
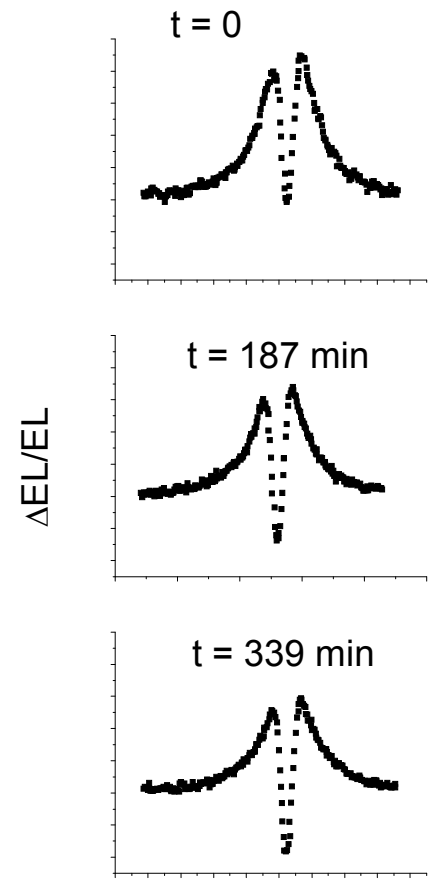
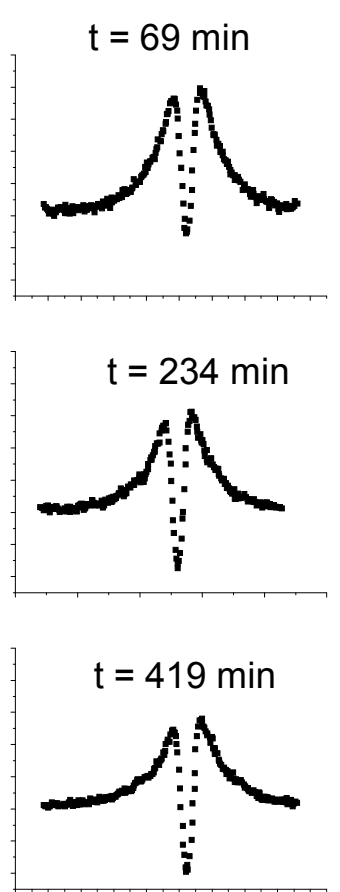

$\mathrm{H}$
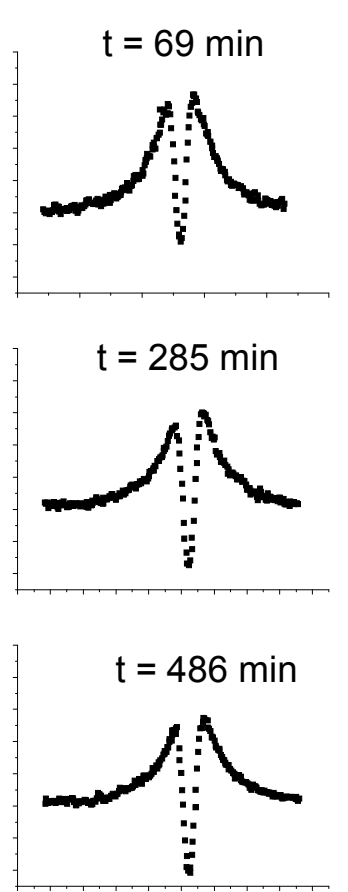

Figure 3.11 Evolution of the spin-1/2 ELDMR in rubrene-doped Alq ${ }_{3}$ devices at 
constant $j$. All curves are plotted on the same scale for better comparison.

Figure 3.12 shows a similar experiment, only this time, the device was driven at constant voltage for 4 hours and then under reverse bias for $30 \mathrm{~min}$. As seen, this reverse bias partially recovers the positive resonance and also suppresses the negative resonance.
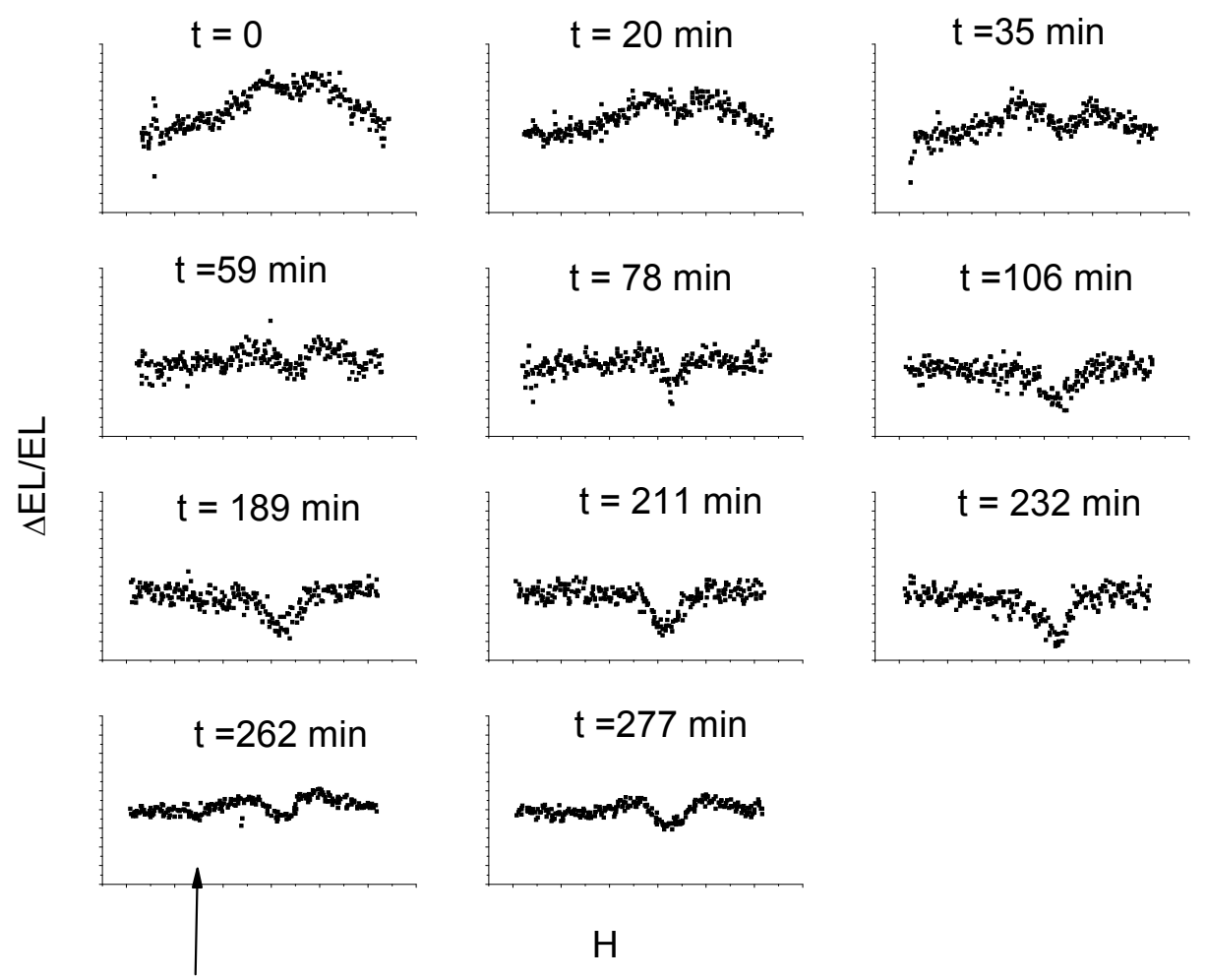

After reverse biased at $5 \mathrm{~V}$ for 30 mins

Figure 3.12 Evolution of the spin-1/2 ELDMR in rubrene-doped Alq 3 devices driven at constant voltage. A reverse bias was applied to the device after $t=232 \mathrm{~min}$ for 30 min. The last two plots show the partial recovery of the positive spin 1/2 ELDMR.

Figure 3.13 shows the same experiment on a similar device driven at constant $j$. The reverse bias has no effect on the ELDMR. Unlike previous degradation experiments, the devices were not protected with flowing nitrogen in these two 
experiments. As seen, the degradation is much faster than in protected devices.

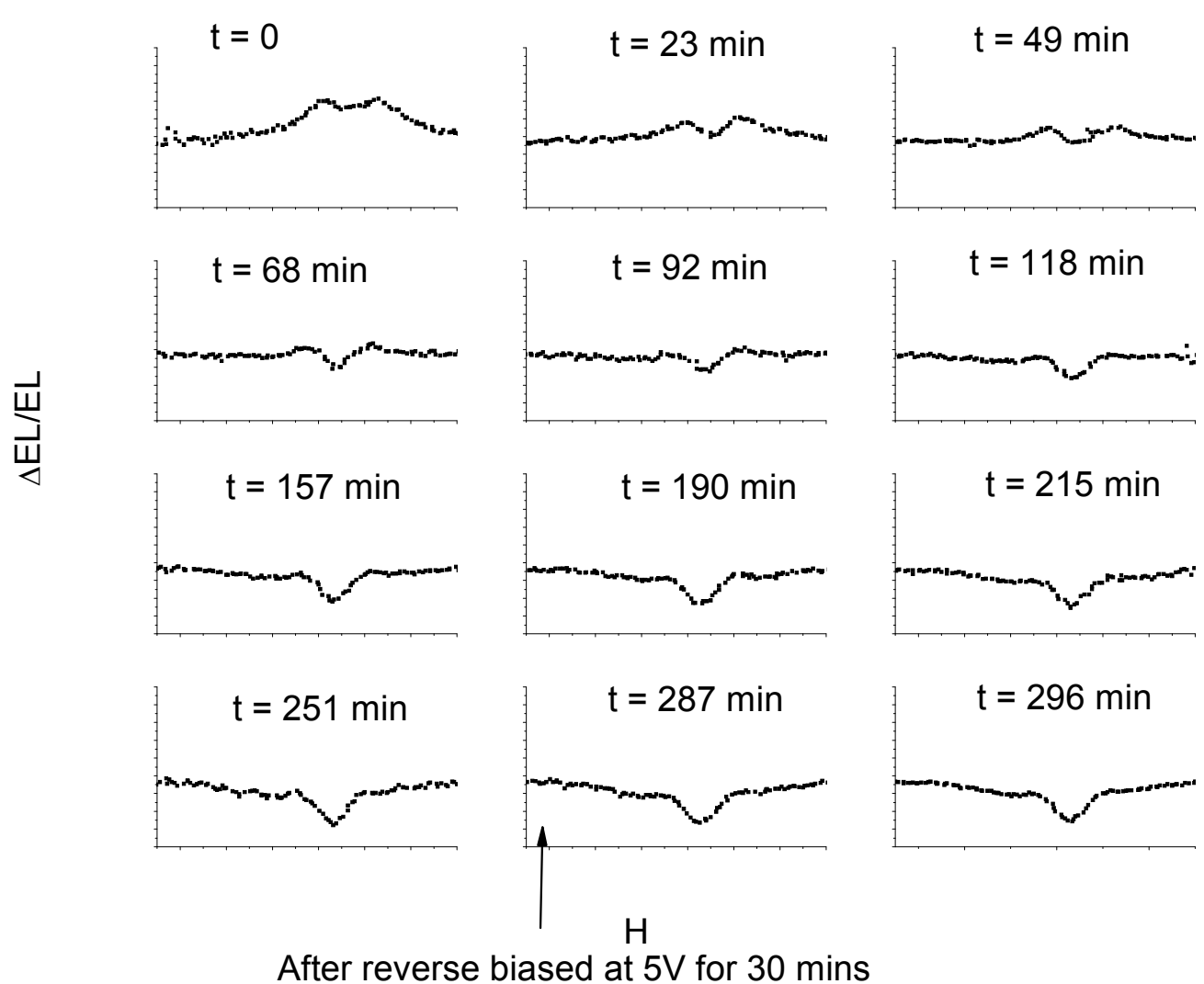

Figure 3.12 Evolution of spin-1/2 ELDMR in Rubrene doped Alq3 device driven by constant current. A reverse bias was applied to the device at 3:05pm. The last two plots show no recovery effect.

Recoverable short-term degradation of OLEDs has been known for a long time [36. 37]. It has been suggested that the mechanism could be a combination of several effects, such as special electrochemical reaction in solid state, internal field formation via trapped charges, movement of impurity ions, and orientation of dipoles. Here we tentatively assign the recoverable degradation to reorientation of dipoles in the device that effectively reduces the internal field, which in turn leads to lower effective current and thus weaker positive resonance and stronger negative resonance. By 
applying reverse bias, the degradation is partially recovered. In constant current driving mode, the effective current remains the same before and after the reverse bias, so no effect could be seen.

\subsubsection{Conclusion}

To conclude, the spin-1/2 ELDMR in rubrene-doped Alq 3 -based devices shows both a quenching component which weakens with increasing $j$ and a positive component which strengthens with $j$. The quenching component is assigned to the enhanced formation of bipolarons under resonance conditions. The enhancing component, together with the triplet powder patterns, is assigned to the TPQ mechanism. The behavior of the ELDMR during degradation, and in particular during recoverable degradation, suggests that the latter is due to orientation of dipoles that reduce the field inside the active layers and consequently $j$ as well.

\section{References}

[1] H. Aziz et al., Chem. Mater. (2004), 16, 4522-4532

[2] P. E. Burrows et al., Appl. Phys. Lett., (1994), 652922

[3] J. McElvain et al., J. Appl. Phys., (1996), 80, 6002

[4] Y. Liew et al., Appl. Phys. Lett., (2000), 77, 2650

[5] Y. Kim et al., Appl. Phys. Lett., (2003), 82, 2200

[6] Y. Sato et al., Cryst. Liq. Cryt., (1994), 253, 243

[7] S. T. Lee et al., Appl. Phys. Lett., (1999), 75, 1404

[8] H. Aziz et al., Science, (1999), 283, 1900

[9] U. Niedermeier et al., Proc. SPIE, (2008), 6999, 699913 
[10] T. D. Nguyen et al., Phys. Rev. B, (2008), 77, 235209

[11] Z. Zhou et al., Adv. Funct. Mater., (2007), 17, 3530

[12] G. Li et al., Phys. Rev. B, (2004), 69, 165311

[13] W. Wagemanns et al., Phys. Status Solidi b, (2010), 1-13

[14] M. A. Baldo et al., Nature, (1998), 395, 151

[15] C. Adachi et al., Appl. Phys. Lett., (2000), 77, 904

[16] H. Murata et al., Proc. SPIE, (1998), 3476, 88

[17] D. J. Fatemi et al., Synth. Met., (1997), 85, 1225

[18] A. A. Shoustikov et al., IEEE J. Select. Topics in Quantum Electronics, (1998), 4, 3

[19] G. Sakamoto et al., Appl. Phys. Lett., (1999), 75, 1682

[20] G. Li, J. shinar, Appl. Phys. Lett., (2003), 835359

[21] J. P. Yang et al., Chem. Phys. Lett., (2000), 325, 251

[22] Z. D. Popovic et al., Thin Solid Films, (2000), 363, 6

[23] Y. Hamda et al., Appl. Phys. Lett., (1999), 75, 1682

[24] H. Y. Kang et al., Mat. Sci. and Engineering C, (2004), 24, 229

[25] Wolfgang Brütting, Physics of Organic Semiconductors, page 287

[26] A. Kadashchuk et al., Phys. Rev. B, (2007), 76, 235205

[27] M. Segal et al., Phys. Rev. B, (2005), 71, 245201

[28] M. K. Lee et al., Phys. Rev. Lett., (2005), 94, 137403

[29] M. Wohlgnannt et al., Nature (London), (2001), 409, 494

[30] L. S. Swanson et al., Phys. Rev. Lett., (1990), 65, 1140 
[31] L. S. Swanson et al., Phys. Rev. B, (1991), 44, 10617

[32] L. S. Swanson et al., Phys. Rev. B, (1992), 46, 15072

[33] E. J. W. List et al., Phys. Rev. B, (2002), 66, 235203

[34] J. A. Weil, Electron Paramagnetic Resonance (Wiley, New York, 1994)

[35] E. J. W. List et al., Phys. Rev. B, (2000), 61, 10807

[36] D. C. Zhou et al., J. Appl. Phys., (1998), 37, L1406

[37] T. Yamada et al., Synth. Met., (2000), 111-112, 237 


\title{
Chapter 4. PLDMR Studies on P3HT and MEH-PPV films
}

\begin{abstract}
PLDMR, including DM-PLDMR, was used to study exciton dynamics under photoexcitation in various $\pi$-conjugated materials. We find that the results can be explained only by TPQ model. Thus this model is the only model consistent with all the positive spin-1/2 resonances in the luminescent $\pi$-conjugated materials and OLEDs.
\end{abstract}

\subsection{Double modulation PLDMR studies in P3HT and MEH-PPV films}

\subsubsection{Intrduction}

Extensive efforts have been made to develop OSCs in the past 25 years [1-5]. Although Started with small molecular materials (pigments), semiconducting polymers have been well incorporated into OSCs resulting in remarkable improvements [6-10].

Among the many $\pi$-conjugated semiconducting polymers, poly (3-hexylthiophene) (P3HT) has been widely used as the electron donor in high efficiency polymer solar cells [10-13]. Studying the processes of photogeneration of charge carriers in these materials is important for a proper understanding of their photoconductivity and related phenomena.

ODMR studies, especially PLDMR studies, have been used to investigate photogeneration and related processes in P3HT [14]. Swanson et al. observed a positive spin- $1 / 2$ resonance and a broad triplet powder pattern around $g=2$ resulting 
from $\Delta m_{S}= \pm 1$ transitions among the three triplet sublevels and a triplet half-field powder pattern around $g=4$, resulting from $\Delta m_{S}= \pm 2$ transitions. The narrow positive spin-1/2 PLDMR resonance was ascribed to recombining distant positive and negative polarons [14]. However, the details of this process were not revealed.

A similar narrow positive spin-1/2 PLDMR resonance is also observed in PPV derivatives, in particular MEH-PPV [15-20]. To explain the resonance, two distinct models have been proposed: (i) The spin-dependent recombination model (SDR model) which assigns the enhancement in the PL to enhanced spin-dependent recombination of singlet polaron pairs to SEs [17, 18], and (ii) The triplet-polaron quenching (TPQ) model which assigns this PLDMR to reduced quenching of SEs by a reduced population of TEs and polarons, that reduced population resulting from enhanced spin-dependent quenching of $[19,20]$. Different experiments and quantitative calculations have been carried out to support each model. However, as will be shown in this chapter, the single modulation microwave chopping frequency dependence of this resonance is not sufficient to support any model. DM-PLDMR provides strong evidence supporting the TPQ model and disqualifying the SDR model.

\subsubsection{Experimental}

The single and double modulated PLDMR experimental setups were the same as described in Chapter 2.

The sample was prepared by evaporating P3HT and MEH-PPV films from toluene solvents onto the inner walls of a glass capillary that was then evacuated and 
sealed. The sample was illuminated by the $\lambda=488 \mathrm{~nm}$ line of an $\mathrm{Ar}^{+}$laser with a intensity of $1000 \mathrm{~mW} / \mathrm{cm}^{2}$ and subjected to spin-1/2 resonance conditions at $\mathrm{X}$ band ( 9.35 GHZ), at various temperatures.

For the single modulation PLDMR, the microwave field was square-wave modulated at $\omega_{M}$. The $\omega_{M}$ component of the PL from the sample was then measured by a lock-in amplifier. For DM-PLDMR, in addition to this microwave modulation, the $\mathrm{Ar}^{+}$laser was sinusoidally modulated at $\omega_{L}$. Two lock-in amplifiers were used in series as described in Chapter 2.

\subsubsection{Results and discussions}

Figure 4.1 shows a typical PLDMR spectrum in P3HT film around $g=2$ at $T=$ $20 \mathrm{~K}$. As seen, a narrow positive spin-1/2 resonance signal is observed at $g=2.0019$, which is consistent with the observations reported by Swanson et al. [14].

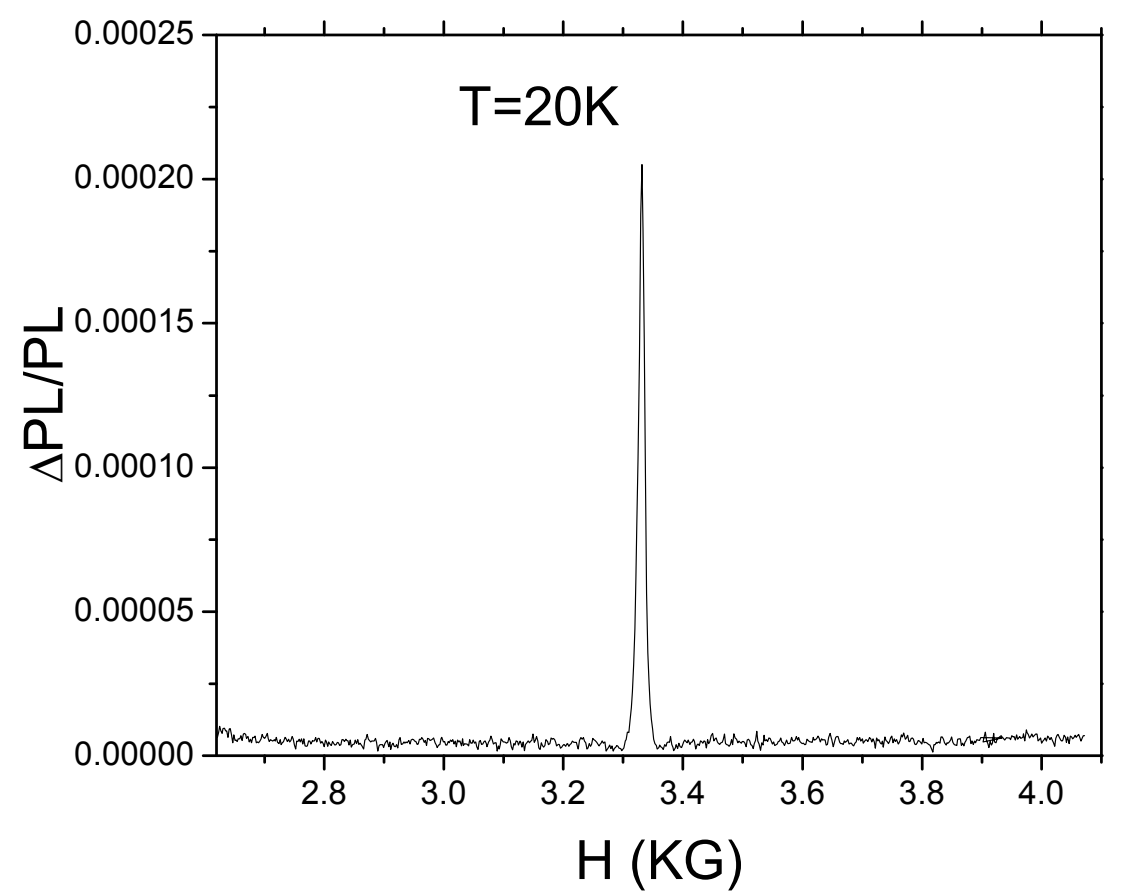

Figure 4.1 Single modulation PLDMR in P3HT film. $\mu$-wave power $P_{\mu}=160 \mathrm{~mW}$, 


$$
\text { Laser power } P_{L}=10 \mathrm{~mW} / \mathrm{mm}^{2}, T=20 \mathrm{~K}
$$

A closer look at the same spectrum but with higher excitation power $P_{L}=40 \mathrm{~mW} / \mathrm{mm}^{2}$ reveals a triplet powder pattern around $g=2$, as shown in Figure 4.2. There are steps at $H \cong(h v \pm D) / g \mu_{B}$ and weak singularities near $H \cong\left(h v \pm D / 2-D^{2} / 8 h v\right) / g \mu_{B}$, with $D / g \mu_{B} \approx 556 G$.

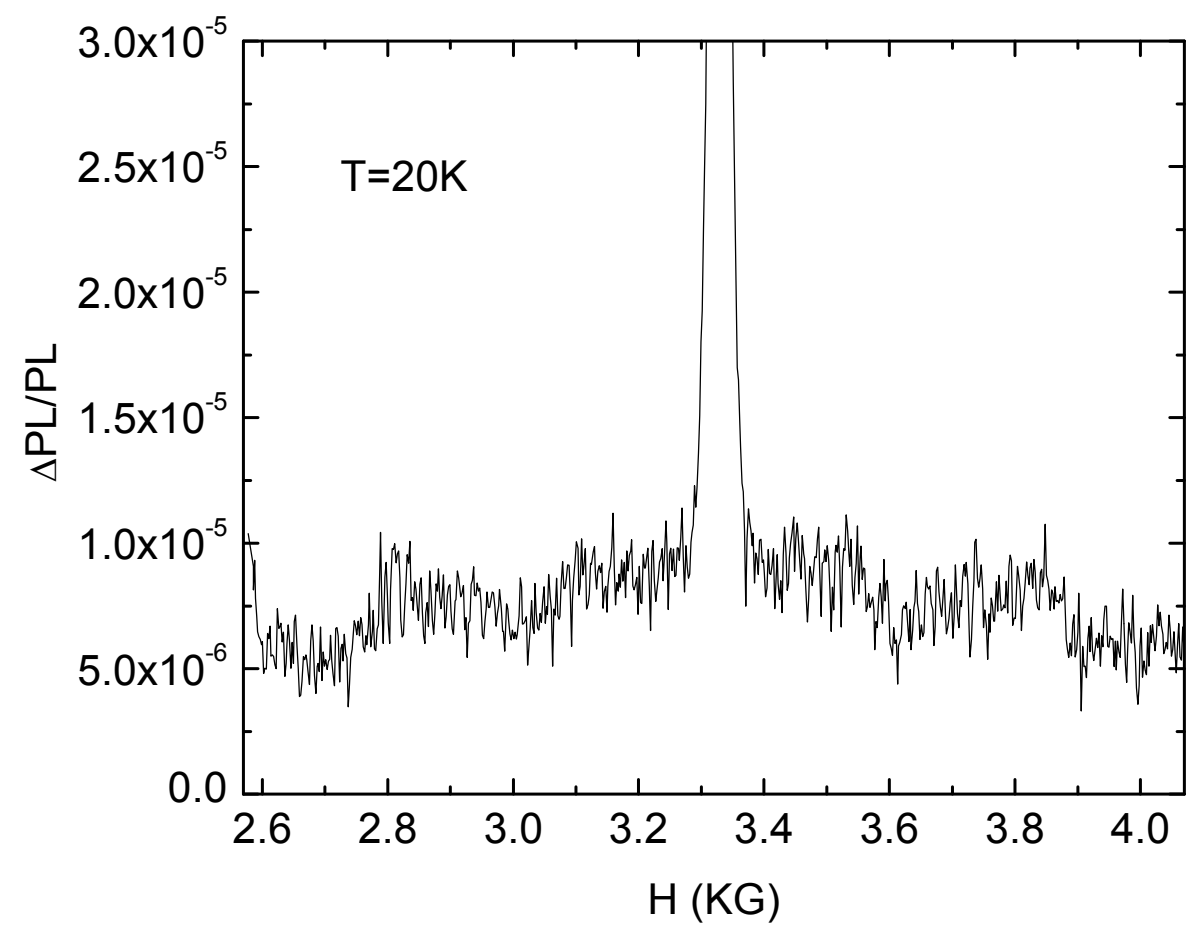

Figure 4.2 Triplet powder pattern PLDMR of $\Delta m_{S}= \pm 1$ in P3HTfilm $\mu$-wave power $P_{\mu}=160 \mathrm{~mW}$, Laser power $P_{L}=40 \mathrm{~mW} / \mathrm{mm}^{2} . T=20 \mathrm{~K}$

This is also in agreement with the reported observations. Indeed, the lineshape of the triplet powder pattern at $T=20 \mathrm{~K}$ is clearly intermediate to the $T=6 \mathrm{~K}$ and $T=$ $125 \mathrm{~K}$ patterns reported by Swanson et al. [14]: From 6 to $20 \mathrm{~K}$, the steps shift toward $h v / g \mu_{B}$, indicating a decreasing $D$. The quenching signal at lower temperature becomes weaker and forms only a dip in the positive resonance. The singularities also 
get weaker, and the broad singularity at the center begins to emerge.

A positive half-field resonance is also observed with temperature dependence similar to the reported results, as shown in Figure 4.3.

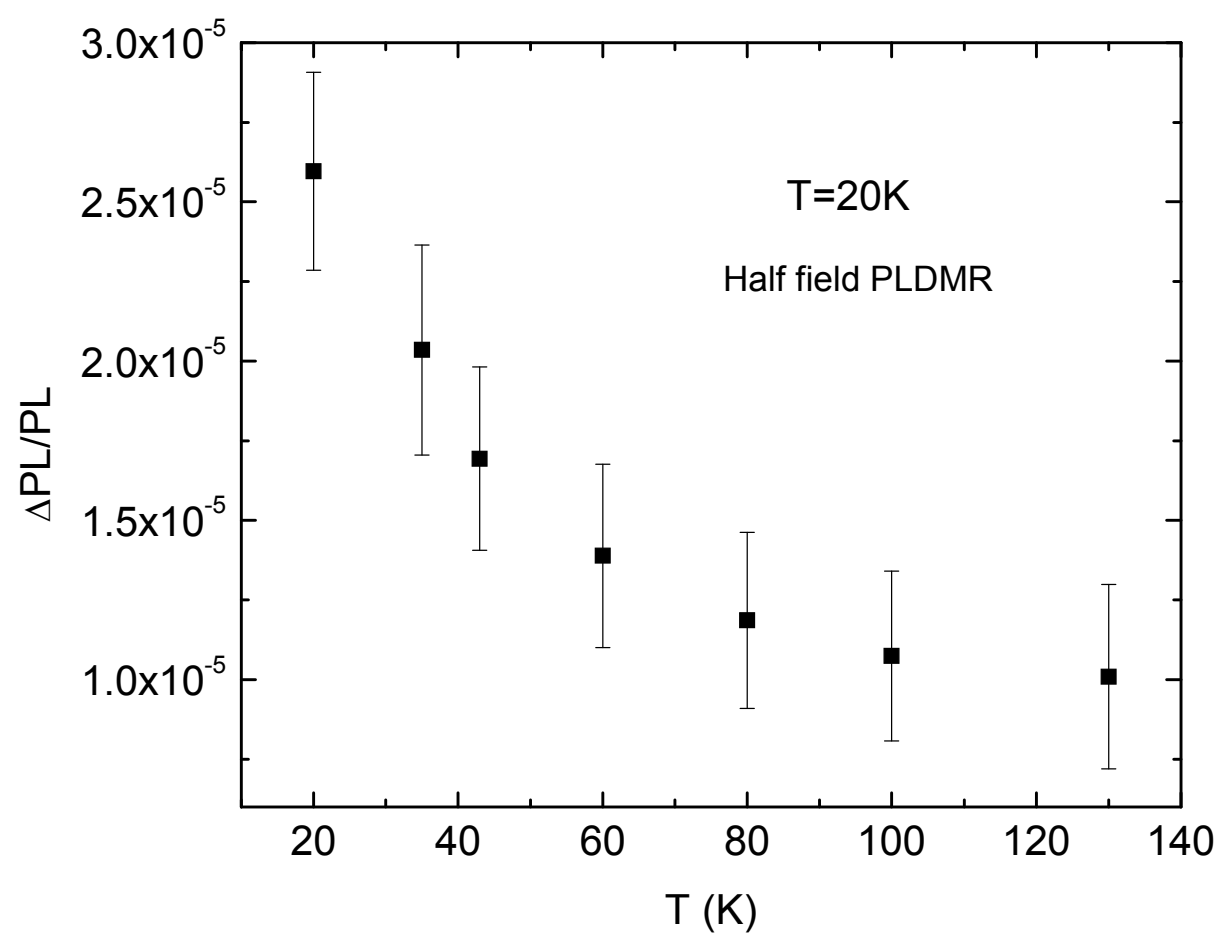

Figure 4.3 The temperature dependence of the amplitude of the half-field $\Delta m_{S}= \pm 2$ PLDMR in P3HT films.

$\mu$-wave power $P_{\mu}=160 \mathrm{~mW}$, Laser power $P_{L}=40 \mathrm{~mW} / \mathrm{mm}^{2} . T=20 \mathrm{~K}$

Figure 4.4 shows a typical DM-PLDMR spectrum around $g=2.0019$ at $f_{L}=100 \mathrm{KHz}$. The positive resonance at such a high $f_{L}$ is still relatively clear. This observation clearly disqualifies the SDR model, as pointed out in references 19 and 20. The amplitude of the DM-PLDMR vs $f_{L}$ at $T=20 \mathrm{~K}$ and $T=35 \mathrm{~K}$ is shown in Fig. 4.5. As seen, in both cases the amplitude of the resonance is essentially independent of $f_{L}$. This clearly shows that the narrow positive spin-1/2 PLDMR is consistent with 
the TPQ model and disqualifies the SDR model.

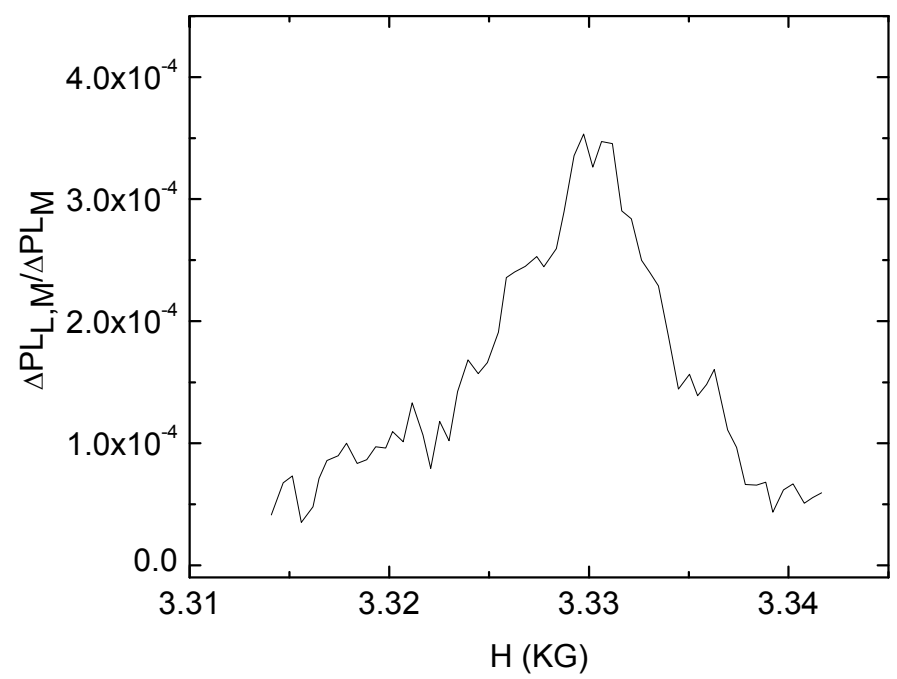

Figure 4.4 A double modulated PLDMR spectrum around $g=2$ in P3HT film.

Modulation frequency $f_{L}=10 \mathrm{KHz}$.

$\mu$-wave power $P_{\mu}=160 \mathrm{~mW}$, Laser power $P_{L}=10 \mathrm{~mW} / \mathrm{mm}^{2} . T=20 \mathrm{~K}$
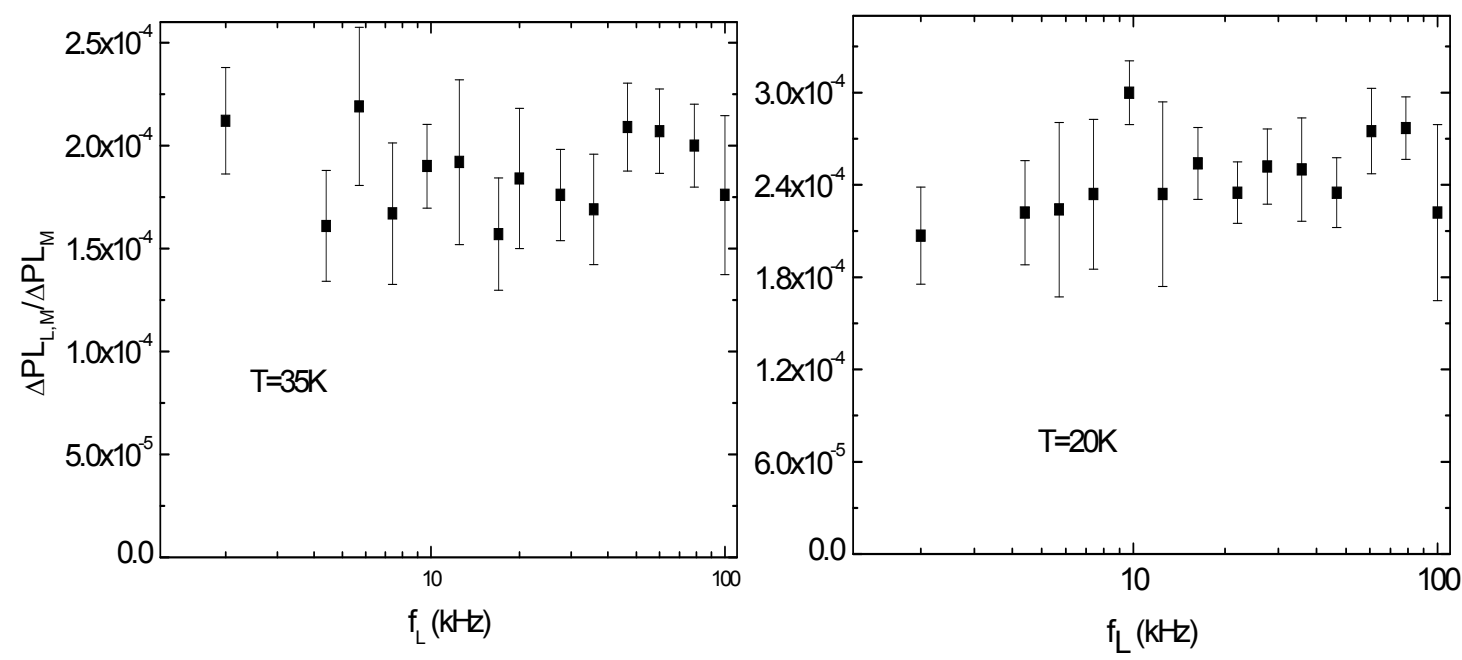

Figure 4.5 Frequency dependence of spin-1/2 DM-PLDMR in P3HT film.

(a) $\mu$-wave power $P_{\mu}=160 \mathrm{~mW}$, Laser power $P_{L}=10 \mathrm{~mW} / \mathrm{mm}^{2} . T=35 \mathrm{~K}$

(b) $\mu$-wave power $P_{\mu}=160 \mathrm{~mW}$, Laser power $P_{L}=10 \mathrm{~mW} / \mathrm{mm}^{2}$. $T=20 \mathrm{~K}$ 
As mentioned in Sec. 4.1.1, the behavior of the positive spin-1/2 PLDMR in MEH-PPV films at $T=20 \mathrm{~K}$ was similarly found to be consistent with the TPQ model. In P3HT, the main narrow positive spin $1 / 2$ resonance, the $\Delta m_{S}= \pm 2$ TE PLDMR, and the broad $\Delta m_{S}= \pm 1$ TE pattern all decrease similarly with increasing $T$. However, in MEH-PPV, unlike the triplet powder pattern which weakens to an unobservable level at $T>100 \mathrm{~K}$, the positive spin-1/2 resonance is observable even at room temperature. It is therefore necessary to examine the DM-PLDMR behavir of this spin-1/2 resonance at various temperatures.
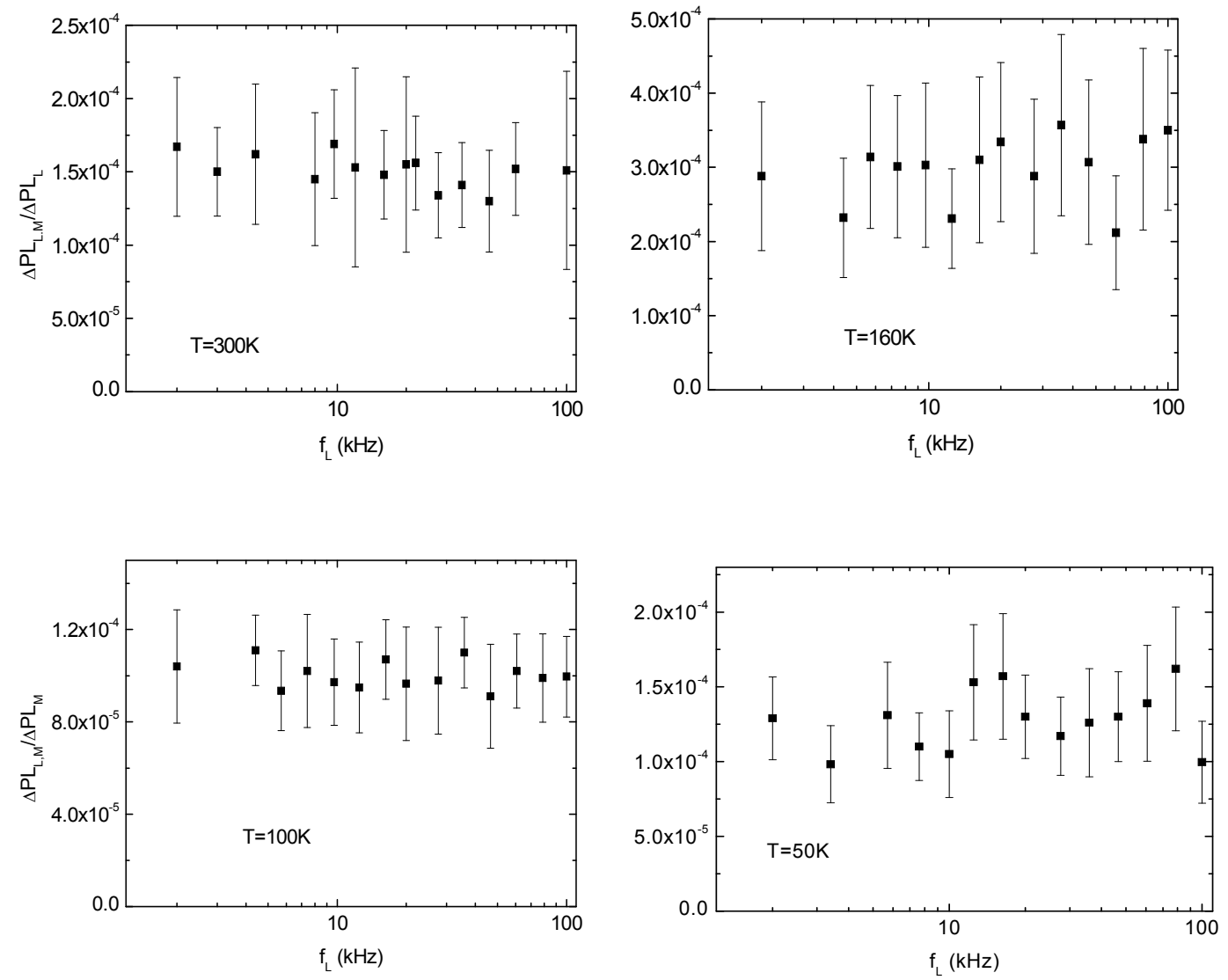

Figure 4.6 DM-PLDMR vs $f_{L}$ in MEH-PPV film at various temperatures: (a) $T=300 \mathrm{~K}$, (b) $T=160 \mathrm{~K}$, (c) $T=100 \mathrm{~K}$ and (d) $T=50 \mathrm{~K}$. $\mu$-wave power $P_{\mu}=810 \mathrm{~mW}$, Laser power $P_{L}=10 \mathrm{~mW} / \mathrm{mm}^{2}$. 
Figure 4.6 shows the DM-PLDMR results in MEH-PPV films vs $f_{L}$ at various temperatures $T>20 \mathrm{~K}$. As seen, the behavior is flat throughout the whole temperature range. Together with the single modulated PLDMR results at different temperatures, the DM-PLDMR results clearly suggest that the narrow positive spin-1/2 PLDMR in MEH-PPV should be explained with TPQ model at all temperatures.

\subsubsection{Conclusion}

To conclude, DM-PLDMR measurements have been carried out in P3HT films and MEH-PPV films. The laser modulation frequency dependence strongly validates the TPQ model and invalidates the SDR model for explaining the narrow positive spin-1/2 PLDMR at $g \sim 2.0025$.

\subsection{Discussions On Single Modulated PLDMR}

\subsubsection{Introduction}

ODMR has been proved to be a powerful tool to investigate exciton dynamics in $\pi$-conjugated materials and OLEDs [22]. This technique is sensitive to population changes in spin sublevels of spin-nonzero species in the materials and thus is able to provide insight into these species and their dynamics. To extract useful information from the results, especially to extract parameters through quantitative simulation, one must identify the mechanisms responsible for the resonances. However, although some resonance mechanisms have been established, such as the mechanism for the quenching spin 1/2 ELDMR and EDMR in OLEDs [21, 23], others are still under debate, notably the mechanism behind the narrow positive spin-1/2 PLDMR in luminescent $\pi$-conjugated polymers. Ever since the SDR and TPQ models were 
proposed, the debate on which model is valid has persisted [17-20, 24, 25]. In particular, Yang et al. analyzed the $f_{M}$ dependence of the separate in-phase and quadrature components of the spin-1/2 PLDMR and concluded that the SDR model accounts for the $f_{M}$ dependence [18] while ignoring the fact that it is inconsistent with the behavior of the DM-PLDMR. The same authors also claimed that the TPQ model cannot explain the frequency dependence if one considers the in-phase and quadrature components respectively instead of considering the total amplitude of the resonance [24].

In the following section, the $f_{M}$ dependence is analyzed within the TPQ model and shown to be consistent with it.

\subsubsection{Quantitative analysis based on the TPQ model}

According to reference 19, the spin-1/2 PLDMR amplitude at $\omega_{M}$ is:

$\left.\Delta P L\right|_{\omega_{M}}=\left|S^{0,1}\right|=G_{S} \tau_{S}\left|-\tau_{S} \gamma_{S T} T^{0,1}-P^{0,1}\left(\tau_{S} \gamma_{S P}+\frac{s}{G_{S}} \chi_{S}\right)\right|$

where $S^{0,1}, T^{0,1}$, and $P^{0,1}$ are the Fourier coefficients of the SE population $S(t)$, TE population $T(t)$, and paired polaron population $P(t)$, respectively. $G_{S}$ is the $\mathrm{SE}$ formation rate, $\tau_{S}$ is the SE lifetime, and $\chi_{S}$ is the fraction of SEs generated from polaron pairs, which is assumed to be a constant 0.25. $\gamma_{S T}$ and $\gamma_{S P}$ are the interaction rates of SEs with TEs and paired polarons, respectively, and $s=i \omega$.

The rate equations for TEs and polarons under the TPQ model are:

$\frac{d P}{d t}=-\frac{P}{\tau_{P}}-\gamma_{T P} T P+G_{P}$ 


$$
\frac{d T}{d t}=-\frac{T}{\tau_{T}}+\left(1-\chi_{S}\right)\left(\frac{P}{\tau_{P}}+\frac{U}{\tau_{U}}\right)-\chi_{S} \gamma_{T P} T P-\gamma_{T P} T U+G_{T}
$$

where $\tau_{T}, \tau_{P}$ and $\tau_{U}$ are the TE, paired polaron and unpaired polaron lifetimes, respectively, $G_{P}$ and $G_{T}$ are the formation rates of paired polarons and TEs, respectively, $\gamma_{T P}$ is the interaction rate of TEs and paired polarons, and $U=U(t)$ is the density of unpaired polarons.

The TPQ model describes the quantities $P, T$ and $\gamma_{T P}$ as changing under resonance. Labeling the derivative with respect to $X$ of the right hand side of the rate equation of $Y$ as $Y_{X}$, we have:

$$
\begin{aligned}
& \frac{P^{0,1}}{\gamma^{0,1}}=\frac{s P_{\gamma}-T_{T} P_{\gamma}+T_{\gamma} P_{T}}{s^{2}+s\left(-T_{T}-P_{P}\right)+\left(P_{P} T_{T}-T_{P} P_{T}\right)} \\
& \frac{T^{0,1}}{\gamma^{0,1}}=\frac{s T_{\gamma}-P_{P} T_{\gamma}+P_{\gamma} T_{P}}{s^{2}+s\left(-T_{T}-P_{P}\right)+\left(P_{P} T_{T}-T_{P} P_{T}\right)}
\end{aligned}
$$

The poles in the equations above are the same. Thus combining equation 4.1, 4.4, and 4.5, one can generalize the solution for the single modulation PLDMR to be:

$$
\left.\Delta P L\right|_{\omega_{M}} / P L=c_{M} \frac{\left(s+z_{M}\right)}{\left(s+p_{M 1}\right)\left(s+p_{M 2}\right)}
$$

where $c_{M}$ is a scaling factor, $p_{M 1}$ and $p_{M 2}$ are the two poles, and $z_{M}$ is the zero. In reference 19, the best fitting gives $p_{M 1}=4100 \mathrm{rad} / \mathrm{s}, p_{M 2}=41400 \mathrm{rad} / \mathrm{s}$, and $z_{M}=$ $5150 \mathrm{rad} / \mathrm{s}$.

One should note that the right hand side of equation 4.6 is complex. Thus setting $c_{M}$ $=1$, the in-phase and quadrature components $x$ and $y$ can be written as:

$$
x=\frac{z_{m} \cdot\left(p_{m 1} \cdot p_{m 2}-\omega^{2}\right)+\omega^{2} \cdot\left(p_{m 1}+p_{m 2}\right)}{\left(p_{m 1} \cdot p_{m 2}-\omega^{2}\right)^{2}+\left(\omega \cdot\left(p_{m 1}+p_{m 2}\right)\right)^{2}}
$$


$y=\frac{\left(p_{m 1} \cdot p_{m 2}-\omega^{2}-z_{m} \cdot\left(p_{m 1}+p_{m 2}\right)\right) \cdot \omega}{\left(p_{m 1} \cdot p_{m 2}-\omega^{2}\right)^{2}+\left(\omega \cdot\left(p_{m 1}+p_{m 2}\right)\right)^{2}}$

Using the values for the parameters as above, the simulated $f_{M}$ dependence of the single modulation PLDMR is shown in Figure 4.7. Note that the in-phase $x$ component is positive throughout the whole frequency range.

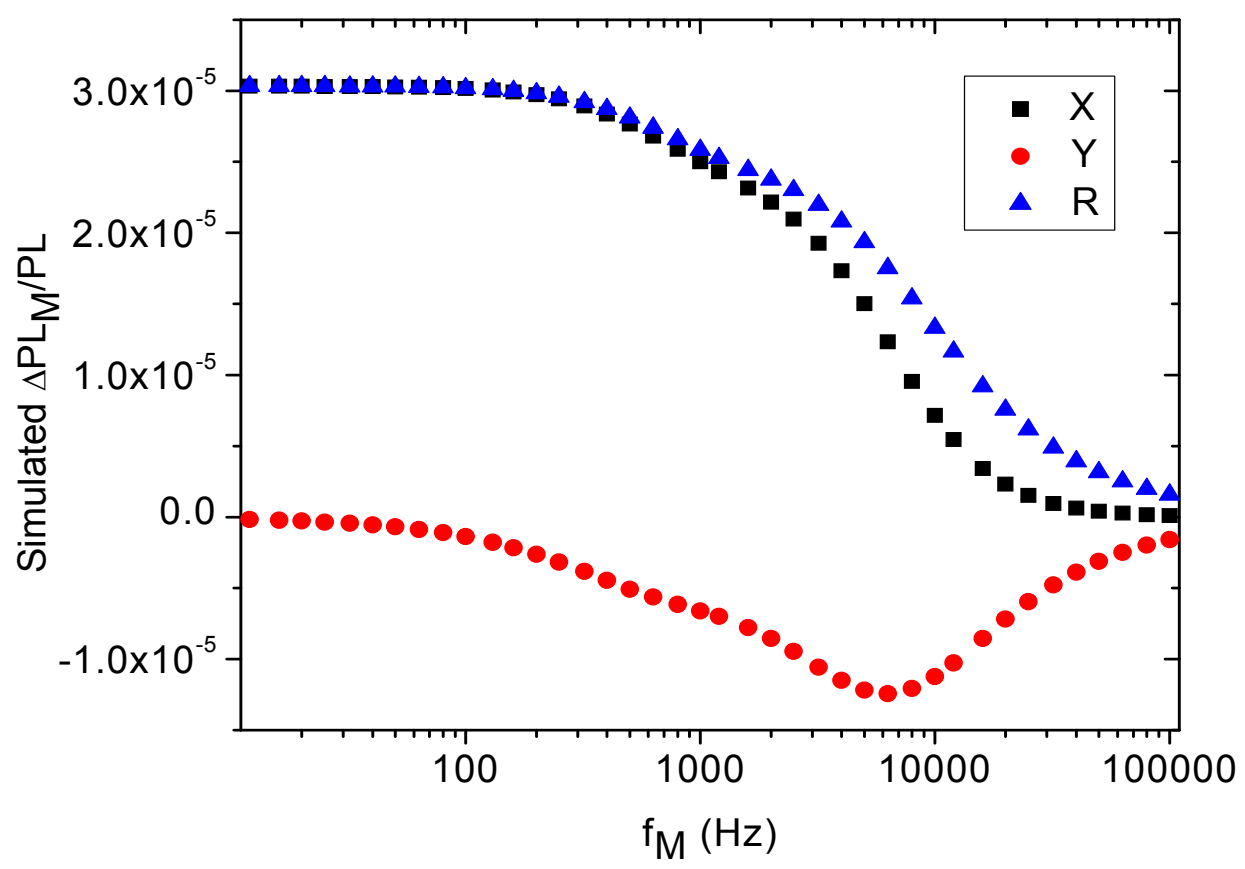

Figure 4.7 Frequency dependence of the in-phase $X$ component, quardature $Y$ component and amplitude $R$ of the single modulation PLDMR simulated by the TPQ model with $p_{M 1}=4100 \mathrm{rad} / \mathrm{s}, p_{M 2}=41400 \mathrm{rad} / \mathrm{s}$, and $z_{M}=5150 \mathrm{rad} / \mathrm{s}$ [19].

Figure 4.8 shows the observed behavior of the room temperature PLDMR in MEH-PPV films. As clearly seen, the $X$ component changes sign at $f_{M}>50 \mathrm{kHz}$. This conflict between the TPQ model prediction and the experimental results led to the conclusion by Yang et al [24] that the TPQ model is invalid, but the SDR model is valid, in explaining the $f_{M}$ dependence of the positive spin-1/2 PLDMR. 


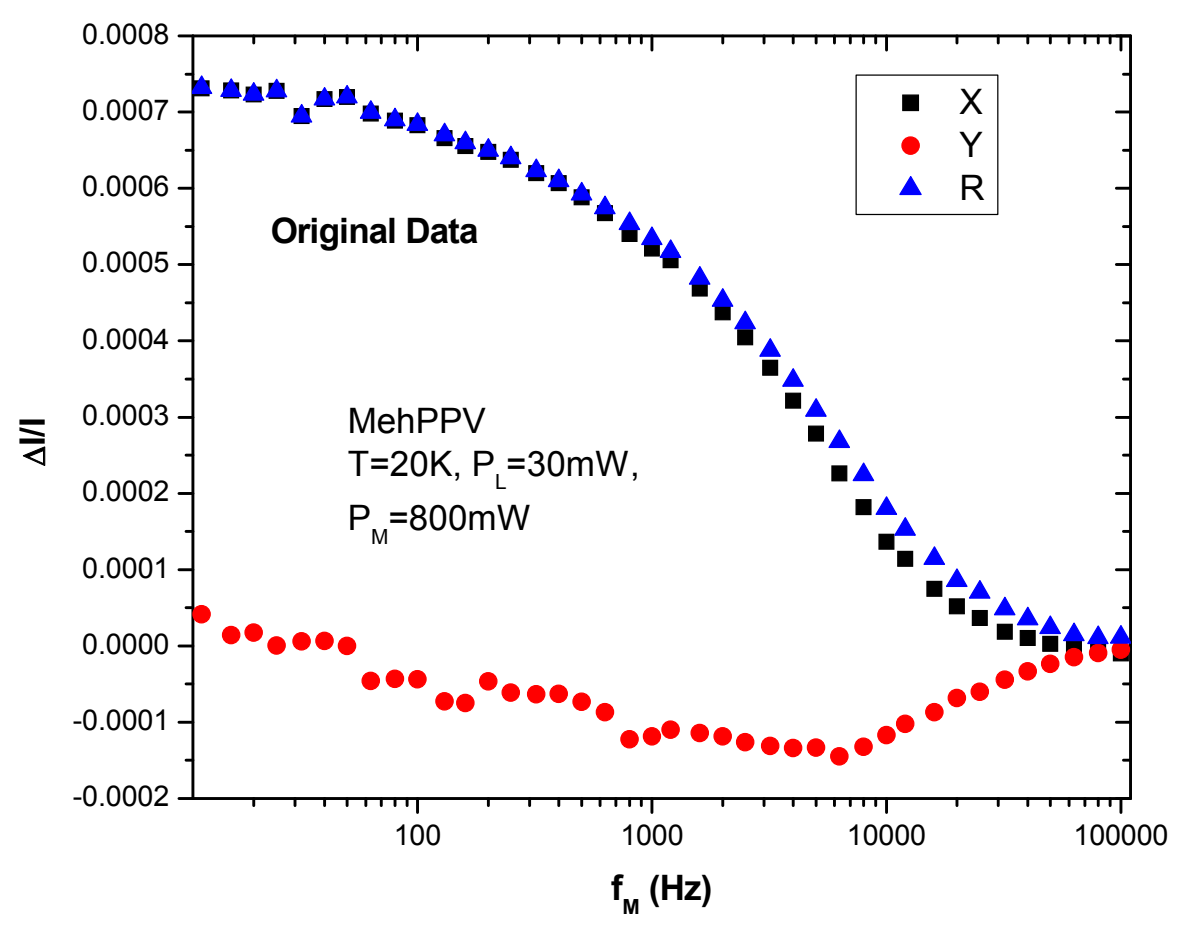

Figure 4.8 Experimental $f_{M}$ dependence of the in-phase $X$ component, quadrature $Y$ component, and amplitude $R$ of the single modulation positive spin 1/2 PLDMR in MEH-PPV films. $\mu$-wave power $P_{\mu}=810 \mathrm{~mW}$, Laser power $P_{L}=30 \mathrm{~mW} / \mathrm{mm}^{2}, T=20 \mathrm{~K}$

Tuning the parameters in the TPQ model to fit both components, agreement with the observed behavior can be obtained as shown in Figure 4.9. Note that both the experimental and simulated behavior are plotted in double logarithmic scales to emphasis the flipping of sign in the in-phase $X$ component. However, since $p_{M 1}$, $p_{M 2}$, and $z_{M}$ should be derived from Eqs. (4.1), (4.4), and (4.5), the values used to simulate the behavior lead to unrealistic TE and polaron liftimes and densities. 

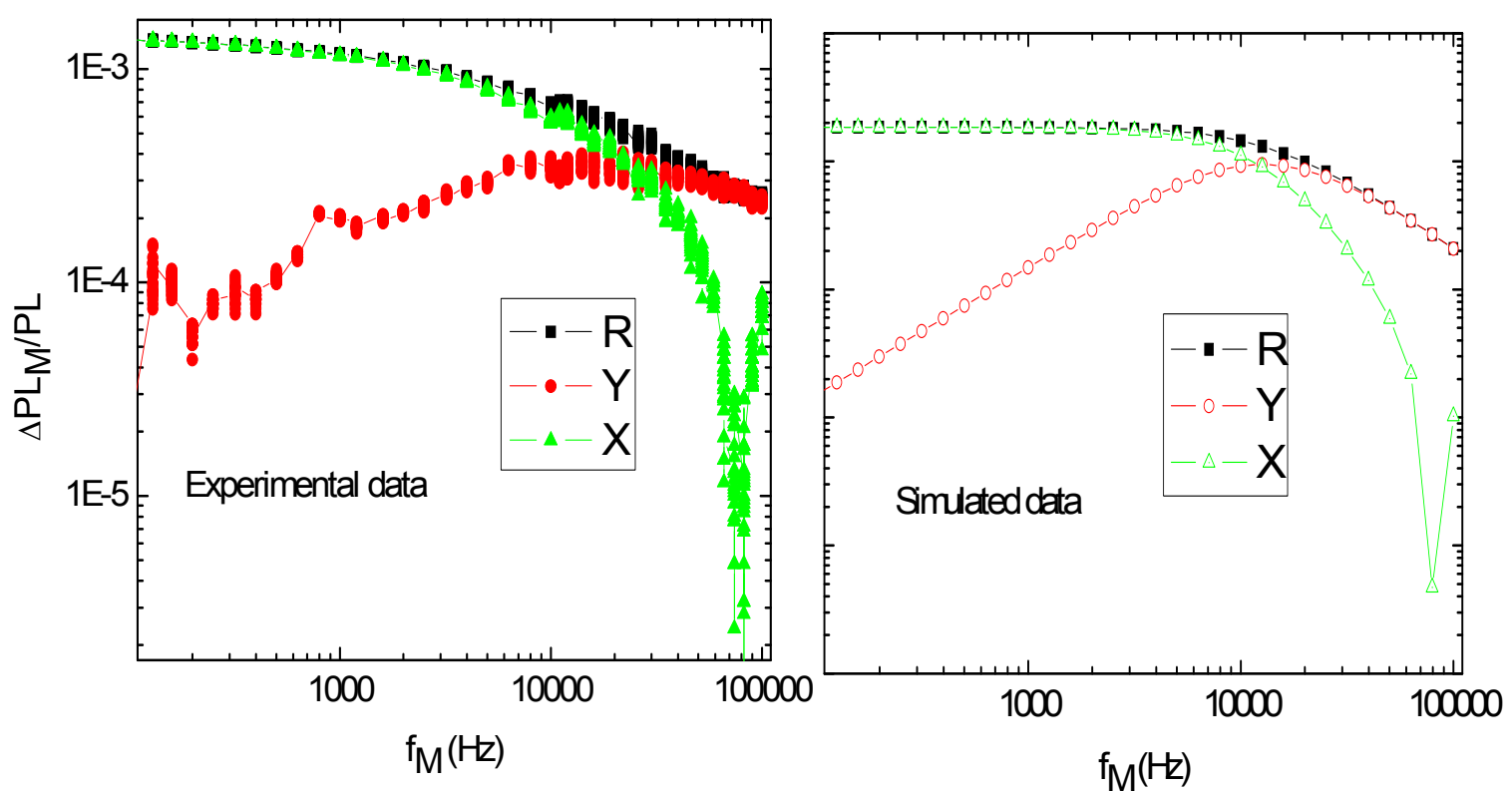

Figure 4.9 Comparison of experimental data and simulated data with tuned values for $p_{M 1}, p_{M 2}$, and $z_{M}$. These values, however, are unrealistic considering the physical meaning of each parameter.

The difficulties in accounting for the observed behavior within the TPQ model originate from one major assumption: That the parameter $c_{M}$ is a scaling factor that is a real number. In examining Eqs. (4.4) and (4.5), one should note that $c_{M}$ is a value proportional to $\gamma^{0,1}$, which is the change in $\gamma_{T P}$ caused by resonance and is not necessarily a real number that would require it to follow the modulation immediately.

Considering the effect of the magnetic resonance on the TPQ process, the enhancement in $\gamma_{T P}$ under resonance conditions actually comes with the following mechanism: Due to the requirement of spin conservation, TEs in TE-polaron (T-P) pairs with total spin $3 / 2$ (i.e., quadruplets) cannot be annihilated by the polaron, in 
contrast to TEs in T-P pairs that have total spin 1/2 (i.e., doublets). Thus for T-P pairs, the lifetimes $\tau_{Q}$ and $\tau_{D}$ for quadruplets and doublets, respectively, must be quite different, i.e., $\tau_{Q}>\tau_{D}$. In addition, the corresponding off-resonance densities $Q$ and $D$ for quadruplets and doublets, respectively, are also different, i.e., $Q>D$. Since only TEs in doublets can be annihilated, the TPQ term in Eq. (4.5), $\gamma_{T P} T(P+U)$, is obviously proportional to the doublet population $D$. Under resonance conditions, there is a net conversion of quadruplets to doublets, thus inducing a new equilibrium for $Q$ and $D$ and changing the annihilation rate. The rate equations for quadruplets and doublets at $T>>h f / k_{B} \cong 0.45 K$ (where $f=9.35 \mathrm{GHz}$ is the microwave frequency) can be written as [26]

$d Q / d t=G_{Q}-\gamma_{Q} Q-(Q-D) P_{\mu w}$

$d D / d t=G_{D}-\gamma_{D} D+(Q-D) P_{\mu w}$

where $\gamma_{Q}=\frac{1}{\tau_{Q}}, \gamma_{D}=\frac{1}{\tau_{D}}$, and $P_{\mu w}$ is the microwave power.

Eqs. (4.9) and (4.10) have the same form as the rate equations in reference 18. It is obvious that the solutions for these equations are frequency-dependent complex numbers that have both in-phase and quadrature components. Under weak signal conditions, i.e., the changes in $T, P$, and $U$ are small compared to the steady state populations, neglecting the $2^{\text {nd }}$ order terms, as mentioned above, we have $\gamma^{0,1} \propto D^{0,1}$. Following the method in reference 19, from Eq. (4.10), we have

$i \omega D^{0,1}=-\frac{1}{\tau_{D}} D^{0,1}+\left(Q_{0}-D_{0}\right) P^{0,1}$ 
Hence $D^{0,1}=\frac{\left(Q_{0}-D_{0}\right) P^{0,1}}{\frac{1}{\tau_{D}}+i \omega}$

where $Q_{0}$ and $D_{0}$ are the steady state populations of quadruplets and doublets off resonance, respectively. Compared to the modulated $P^{0,1}$, the modulated $D^{0,1}$ and thus $\gamma^{0,1}$ both have a phase shift $\theta$ that satisfies the relation

$\tan \theta=-\omega \tau_{D}=-2 \pi f \tau_{D}$

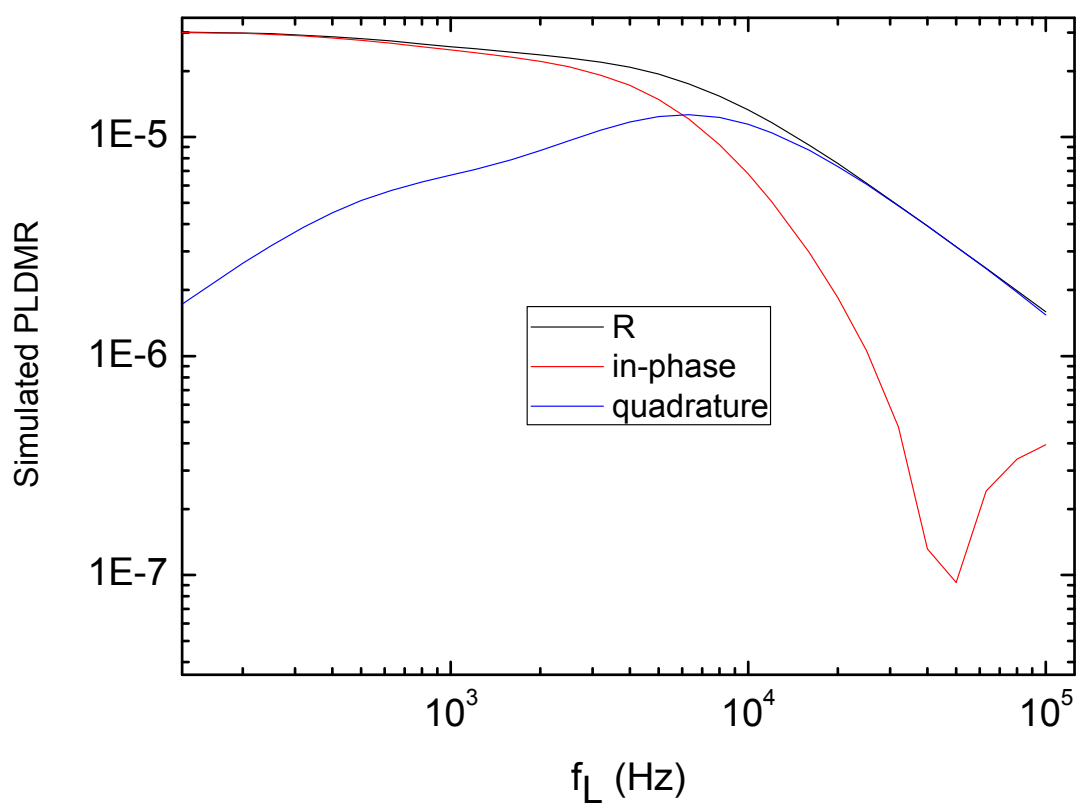

Figure 4.10 Simulated PLDMR under TPQ model which takes in account $\theta$ f the phase

$$
\text { shift in } \gamma_{T P} \text {. }
$$

With $\tau_{D}=500 n s$, we see that the sign of the in-phase signal changes around $f_{L}=50 \mathrm{KHz}$ in the simulation with the TPQ model, as shown in (Figure 4.10). Clearly, the calculations above include numerous approximations. However, they still show that only the TPQ model can account for all of the mateh the experimental results. 


\subsubsection{Conclusion}

Two models have been proposed for explaining the positive spin-1/2 PLDMR in luminescent $\pi$-conjugated materials: the TPQ and the SDR models. The DM-PLDMR results in P3HT and MEH-PPV films are in complete agreement with the TPQ model and in total disagreement with the SDR model. Interestingly, the TPQ model is also capable of explaining the behavior of the in-phase $X$ and quadrature $Y$ components of the single modulation PLDMR. Thus the TPQ model is the only model that fits all the experimental observations.

\section{References:}

[1] G. A. Chamberlain, Solar Cells, (1983), 8, 47

[2] D. Wöhrle et al., Adv. Mater., (1991), 3, 129

[3] C. J. Brabec et al., Adv. Funct. Mater., (2001), 11, 15

[4] J. Nelson et al., Curr. Opin. Solid State Mater. Sci., (2002), 6, 7

[5] H. Hoppe et al., J. Mater. Res., (2004), 19, 7

[6] Handbook of Conducting Polymers, edited by T. A. Skotheim, (1986)

[7] Handbook of Organic Conductive Molecules and Polymers, edited by H. S. Nalwa, (1997)

[8] Semiconducting Polymers, edited by G. Hadziioannou et al., (2000)

[9] C. winder et al., J. Mater. Chem., (2004), 14, 1077

[10] Y. Kim et al., Nature Mater., (2006), 5, 197

[11] G. Li et al., Nature Mater., (2005), 4, 864

[12] T. Xiao et al., Org. Electron., (2011), 12257 
[13] W. Ma et al., Adv. Funct. Mater., (2005), 15, 1617

[14] L. S. Swanson et al., Phys. Rev. Lett., (1990), 65, 1140

[15] V. Dyakonov et al., Phys. Rev. B, (1997), 56, 3852

[16] V. Dyakonov et al., Phys. Rev. B, (1999), 59, 8019

[17] M. Wohlgenannt et al., Phys. Rev. Lett., (2002), 88, 197401

[18] C. G. Yang et al., Phys. Rev. Lett., (2007), 99, 157401

[19] M. Segal et al., Phys. Rev. B, (2005), 71, 245201

[20] M. K. Lee et al., Phys. Rev. Lett., (2005), 94, 137403

[21] G. Li et al., Phys. Rev. B, (2004), 69, 165311

[22] J. Shinar, Optically Detected Magnetic Resonance Studies of Luminescence-Quenching Processes in $\pi$-conjugated Materials and Organic Light-Emitting Devices, to be published in Laser and Photonics Reviews

[23] A. Kadashchuk et al., Phys. Rev. B, (2007), 76, 235205

[24] C. G. Yang et al., Phys. Rev. B, (2007), 75, 246201

[25] C. G. Yang et al., Phys. Rev. Lett., (2006), 96, 089701

[26] E. Lifshitz et al., Annu. Rev. Phys. Chem., (2004), 55, 509 


\title{
Chapter 5. Colossal transient room temperature \\ photocurrent-detected magnetic resonance in organic devices: a magnetic resonance-induced switch
}

\begin{abstract}
Organic spintronics is drawing intense attention due to its potential to revolutionize, inter alia, computing technologies. Yet all magnetic field or related resonance effects reported to date are either small $(<3 \%)$ or significant only at low temperatures. This paper describes, for the first time, a colossal transient room-temperature X-band photocurrent $\left(I_{P C}\right)$-detected magnetic resonance (PCDMR) in single layer poly(2-methoxy-5-(2'-ethyl)-hexoxy-1,4- phenylene vinylene (MEH-PPV) devices, in effect a magnetic resonance switch. The mechanism most likely responsible for this colossal effect is the spin-dependent formation of spinless bipolarons adjacent to negatively-charged deep traps, apparently induced by $\mathrm{O}_{2}$ centers, to form trions.
\end{abstract}

\subsection{Introduction}

Magnetic field effects (MFEs) and optically and electrically detected magnetic resonance (ODMR and EDMR, respectively) in $\pi$-conjugated materials and devices have been studied for over twenty years.[1-16] Recently, however, such studies have gained strong momentum[17-20] due to their potential to spawn a spintronic technology revolution.[21-23] Yet all organic MFEs, ODMR, and EDMR effects reported to date are either small ( $\sim 3 \%$ or less) or significant only at low temperatures.

Poly(2-methoxy-5-(2'-ethyl)-hexoxy-1,4-phenylene vinylene (MEH-PPV) films 
and devices based on it have been widely studied by ODMR and EDMR.[14,15, 24-29] In particular, the photoluminescence (PL)-enhancing (positive) spin-1/2 PL-detected magnetic resonance (PLDMR) of MEH-PPV and other PPV films has been studied extensively.[2,5,7,14-16,19] The amplitude of the resonance, i.e., the fractional change in the PL intensity $\Delta I_{P L} / I_{P L}$, while strong relative to that of other materials, is only $10^{-4}-10^{-3}$ at room temperature. One of the strongest ODMRs or EDMRs reported to date is the photocurrent (PC) detected magnetic resonance (PCDMR) of indium oxide (ITO)/poly(p-phenylphenylene vinylene (PPPV)/Al,[9] where $\Delta I_{P C} / I_{P C} \sim 0.03$ at $77 \mathrm{~K}$.[9]

This paper describes the room temperature PCDMR in single layer ITO/MEH-PPV/Al devices, where $9.35 \mathrm{GHz} \mathrm{X}$-band magnetic resonance conditions induce a colossal transient $\Delta I_{P C} I_{P C}$, in particular in devices where the MEH-PPV film was baked overnight at $100^{\circ} \mathrm{C}$ in pure $\mathrm{O}_{2}$. Interestingly, $\Delta I_{P C}$ is relatively independent of the total steady state photocurrent $I_{P C}$. By comparing the behavior of devices in which the MEH-PPV film was baked overnight at $100^{\circ} \mathrm{C}$ in dry $\mathrm{O}_{2}$ vs those with unbaked films (which were kept in vacuum), it is concluded that the mechanism most likely responsible for this colossal effect is the strongly spin-dependent formation of spinless bipolarons adjacent to negatively-charged deep traps, apparently induced by $\mathrm{O}_{2}$ centers, to form trions.

\subsection{Experimental}

MEH-PPV (average number molecular weight 40,000 $\leq M_{n} \leq 70,000$ ) was 
purchased from Sigma-Aldrich. The polymer was dissolved in chlorobenzene and then spin coated on a Colorado Concept Coatings $\sim 140$ nm-thick $10 \Omega /$ sq ITO-coated glass substrate at $2000 \mathrm{rpm}$. It was then baked at $60^{\circ} \mathrm{C}$ for $30 \mathrm{~min}$ in an Argon-filled glovebox before further treatment. The Al was thermally deposited on the MEH-PPV layer in a vacuum chamber (background pressure $\sim 10^{-6}$ mbar) inside the glovebox.

The samples were placed in the quartz dewar of an Oxford Instruments cryostat inside an optically accessible $9.35 \mathrm{GHz}$ X-band microwave cavity during PCDMR measurements. $I_{P C}$ was excited at $488 \mathrm{~nm}$ by a Spectra-Physics $\mathrm{Ar}^{+}$laser. The microwave-induced $\Delta I_{P C}$ was detected by monitoring the voltage across a standard resistor connected in series with the ITO/MEH-PPV/Al device. For time-resolved measurements that voltage was monitored directly by a digital storage oscilloscope while the microwaves were chopped at $f_{\mu}=50 \mathrm{~Hz}$. For cw measurements that voltage was monitored by a lockin amplifier referenced by $f_{\mu}$, which was varied from $50 \mathrm{~Hz}$ to $10 \mathrm{kHz}$. Since the PCDMR amplitude is sensitive to fabrication variables, all comparisons are made to a reference cell from the same batch so that the uncontrolled fabrication variables are minimized.

Secondary ion mass spectrometry (SIMS) depth profiling of ITO/MEH-PPV/Al devices, using a $4 \mathrm{kV}$ Cs ion beam, was conducted by Evans Analytical Group using a PHI 6300 Quad SIMS system.

\subsection{Results and discussions}

\subsubsection{Colossal PCDMR}

Figure 5.1(a) shows the central result of this work. It compares the time-resolved 
behavior of $I_{P C}$ vs time $t$ at the resonant field $H_{O N}=3,333 \mathrm{G}$ (top trace) and off-resonance $H_{O F F}=3,283 \mathrm{G}$ (middle trace) of ITO/MEH-PPV/Al, with the MEH-PPV film baked overnight in $\mathrm{O}_{2}$. It was observed that baking in $\mathrm{O}_{2}$ greatly strengthens the resonance, as will be discussed in detail below. The bottom trace of Fig. 5.1(a) shows the microwave power vs $t$. Off resonance $I_{P C} \approx-1 \mathrm{nA}$ and $\Delta I_{P C}=0$.
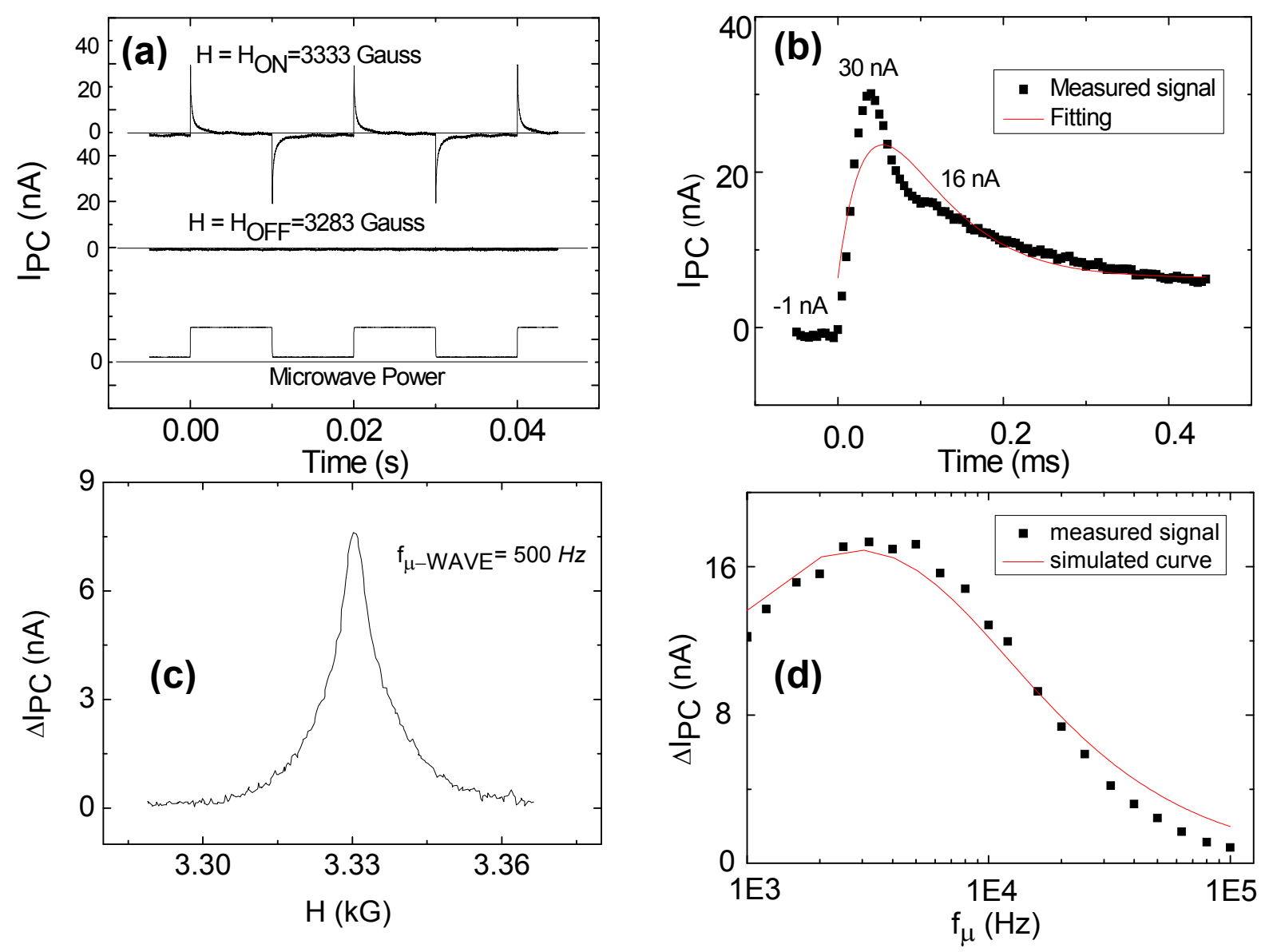

Figure 5.1. (a) $I_{P C} v$ s time t at $0 \leq t \leq 50 \mathrm{~ms}$ at $H_{O N}=3,333 \mathrm{G}$ (upper trace) and $H_{O F F}$ $=3,283 \mathrm{G}$ (middle trace). The lower trace is the microwave power. (b) $I_{P C}$ at $H_{O N} v s t$ at $0 \leq t \leq 400 \mu$ s. the microwaves are turned on at $t=0$. (c) $\Delta I_{P C} v s$ magnetic field observed through a lockin amplifier. The microwave chopping frequency $f_{\mu}=500 \mathrm{~Hz}$.

(d). $f_{\mu}$ dependence of $\Delta I_{P C}$.

However, on resonance a transient $\Delta I_{P C} \neq 0$ is clearly seen. Indeed, as seen in Fig. 
5.1(b), at $t=39 \mu \mathrm{s}, \Delta I_{P C} \approx+30 \mathrm{nA}$, i.e., $\Delta I_{P C} / I_{P C} \approx-30$. This is clearly a colossal value for any magnetic resonance. It is $\geq 3$ orders of magnitude stronger than the strongest $\mathrm{cw}$ or time resolved resonances reported to date. $[9,18]$ In effect, it demonstrates a room temperature magnetic resonance-induced switch.

To reduce noise, lockin detection at $f_{\mu}$ was used in the cw comparisons shown and discussed below. A typical $\mathrm{cw} \Delta I_{P C}$ is shown in Figure 5.1(c). Note that the lockin amplifier output gives only the time-averaged $\Delta I_{P C}$. Thus, as Figure 5.1(d) shows, this reading is $f_{\mu}$-dependent. To obtain a good signal-to-noise ratio at relatively low $f_{\mu}, f_{\mu}$ was kept at $500 \mathrm{~Hz}$ for the results shown below. As seen in Fig. 5.1(c), the resonance peak is at $H_{O N}=3,330.2 \mathrm{G}$ and $g=2.0027 \pm 0.0003$. This $g$ value is similar to that reported for the positive polarons in poly(2-methoxy-5-(3-,7-dimethyloctyloxy)-1,4-phenylenevinylene) (MDMO-PPV):[6,6]- phenyl $\mathrm{C}_{61}$-butyric acid methyl ester (PCBM).[30] In that system, $95 \mathrm{GHz}$ electron paramagnetic resonance (EPR) yielded $g_{\|}=2.0034(1)$ and $g_{\perp}=2.0024(1)$ for the positive polaron on MDMO-PPV, and $g_{x}=2.0003(1), g_{y}=$ 2.0001(1), and $g_{z}=1.9982(1)$ for the negative polaron on PCBM. These values are also consistent with a previous EDMR study on MEH-PPV devices.[27] In that work, a magnetic resonance-induced change in the (dark) conductivity $\sigma$, with $g$-factors between 2.001 and 2.003, were observed. In agreement with earlier and later studies, $[5,7,13,16]$ that EDMR was assigned to the strongly spin-dependent process $p^{+}+p^{+} \rightarrow b p^{++}+$phonons 
where $p^{+}$are positive spin $1 / 2$ polarons (holes) and $b p^{++}$are doubly-charged positive spinless bipolarons. The full-width-at-half maximum (FWHM) observed in this work is $\sim 18 \mathrm{G}$ at a lowered microwave power $P_{\mu}=40 \mathrm{~mW}$ (to avoid microwave-induced broadening), which is also similar to the FWHM $\sim 20 \mathrm{G}$ of the EDMR reported by Silva et al.[27]

Degradation increases $\left|\Delta I_{P C} I_{P C}\right|$, mainly due to the decreased $\left|I_{P C}\right|$. Figures 5.2(a) and 5.2(b) show the time evolution of $\Delta I_{P C}$ and $I_{P C}$, respectively. As seen, both degrade. However, while $\left|I_{P C}\right|$ degrades from $328 \mathrm{nA}$ to $4 \mathrm{nA}$ in $104 \mathrm{hrs}$, a drop of nearly $99 \%, \Delta I_{P C}$ is much more stable, degrading from $4.5 \mathrm{nA}$ to $2.6 \mathrm{nA}$, i.e., by $42 \%$ only, and it actually stabilizes at $2.5 \mathrm{nA}$ after $\sim 40 \mathrm{hrs}$. This increases $\left|\Delta I_{P C} / I_{P C}\right|$ with time, as shown in Figure 5.2(c). After $\sim 104$ hrs, it reaches a value of 0.73, colossal for any magnetic resonance amplitude, and it continues to increase, albeit much more slowly. The independence of $\Delta I_{P C}$ with respect to $I_{P C}$ can be further shown in biased PCDMR measurements. Figures 5.2(d) -5.2(f) show $\Delta I_{P C}, I_{P C}$, and $\Delta I_{P C} I_{P C}$ (the PCDMR) vs the bias $V$, where the Al cathode was grounded and a positive $V$ was applied to the ITO anode. With increasing $V$, up to $0.9 \mathrm{~V}, \Delta I_{P C}$ increases from 2.0 to $5.2 \mathrm{nA}$, while $I_{P C}$ increases from -263 to $+112 \mathrm{nA}$. Thus $\Delta I_{P C} I_{P C}$ actually diverges when $I_{P C}$ approaches zero at the open circuit voltage $V_{O C}$. The degradation effect and especially the bias dependence cannot be explained by enhanced quenching of $I_{P C}$ due to enhanced bipolaron formation, as described in earlier EDMR studies [5-7,13,16,27]. Any $P C$-quenching mechanism would limit $\Delta I_{P C}$ to $\left|I_{P C}\right|$ and thus $\Delta I_{P C}$ would vanish when $I_{P C} \rightarrow 0$, in stark contrast to the observed behavior. Additional evidence that the 
PCDMR observed here originates from a different mechanism, other than enhanced $b p^{++}$formation, is the behavior of the dark EDMR of these devices: Within the same bias range, or a bias that would generate a dark current similar to $\left|I_{P C}\right|$, no dark EDMR was observed, in contrast to the EDMR and PCDMR of PPV PLEDs reported by Swanson et al. [5]. This is not surprising as the previously reported EDMR has a magnitude $<10^{-4}$ in PPV-based devices [5,7,27] which is much smaller than $\Delta I_{P C} / I_{P C}$ observed in this work and too small to be observed using the present PCDMR setup.
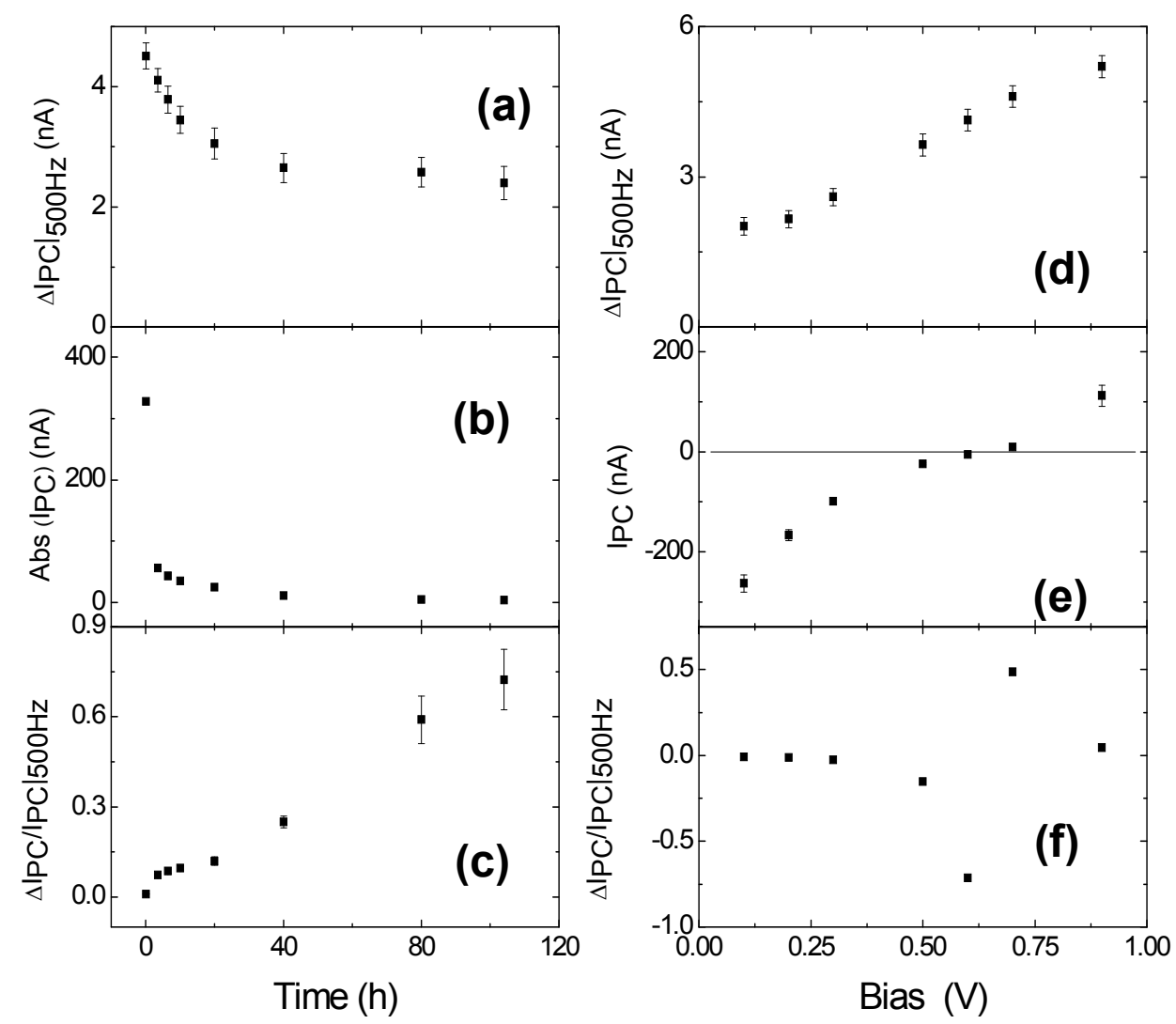

Figure 5.2. (a)-(c) Resonance induced change $\Delta I_{P C}$, off-resonance photocurrent $I_{P C}$, and $\triangle I_{P C} / I_{P C} v$ s time, respectively, when the sample degraded in air. (d)-(f) $\Delta I_{P C}, I_{P C}$, and $\Delta I_{P C} / I_{P C}$, respectively, vs bias. The lines are a guide to the eye. 


\subsubsection{Possible mechanisms}

As mentioned above, it was observed that baking in $\mathrm{O}_{2}$ enhances $\Delta I_{P C}$ strongly. Figure 5.3(a) compares $\Delta I_{P C}$ of devices with and without $\mathrm{O}_{2}$-baking. Following the deposition of the MEH-PPV layer, Device A1 was transferred into a vacuum chamber (pressure $\sim 10^{-6}$ torr) and pumped for $14 \mathrm{~h}$. Device A2 was baked at $100^{\circ} \mathrm{C}$ for $14 \mathrm{~h}$ in flowing dry high-purity oxygen. The Al electrode was then thermally evaporated on both devices, completing their fabrication. As clearly seen in Fig. 5.3(a), Device A2 that was baked exhibits a much stronger $\Delta I_{P C}$ than Device A1. As noted, due to the high sensitivity of the ODMR technique, small variations in the devices could lead to large differences in the ODMR amplitude, so only comparisons within the same batch of devices, with minimal uncontrolled variations, are made.

To understand the role of baking in $\mathrm{O}_{2}$, several mechanisms were examined. First, since $I_{P C}$ can be greatly affected by carrier transport and extraction conditions in the device, $\Delta I_{P C}$ can also be affected by these factors. Additionally, we expect that exposure of the MEH-PPV film to $\mathrm{O}_{2}$ for $14 \mathrm{~h}$ at $100^{\circ} \mathrm{C}$ will result in a higher concentration of oxidized $\mathrm{Al}$ at the $\mathrm{MEH}-\mathrm{PPV} / \mathrm{Al}$ interface, in effect creating an $\mathrm{AlO}_{x}$ buffer layer $[13,31,32]$ which in turn will affect charge carrier transport and extraction. To examine whether this change in carrier transport and extraction is responsible for the behavior of $\Delta I_{P C}$, measurements on devices (all with an MEH-PPV film baked in $\mathrm{O}_{2}$ ) with other buffer layers were performed. In Device B1, a $1 \mathrm{~nm} \mathrm{LiF}$ buffer layer was inserted between the MEH-PPV film and the Al cathode. In Device B2 a $5 \mathrm{~nm}$ $\mathrm{MoO}_{x}$ buffer layer was inserted between the ITO and the MEH-PPV layers. 

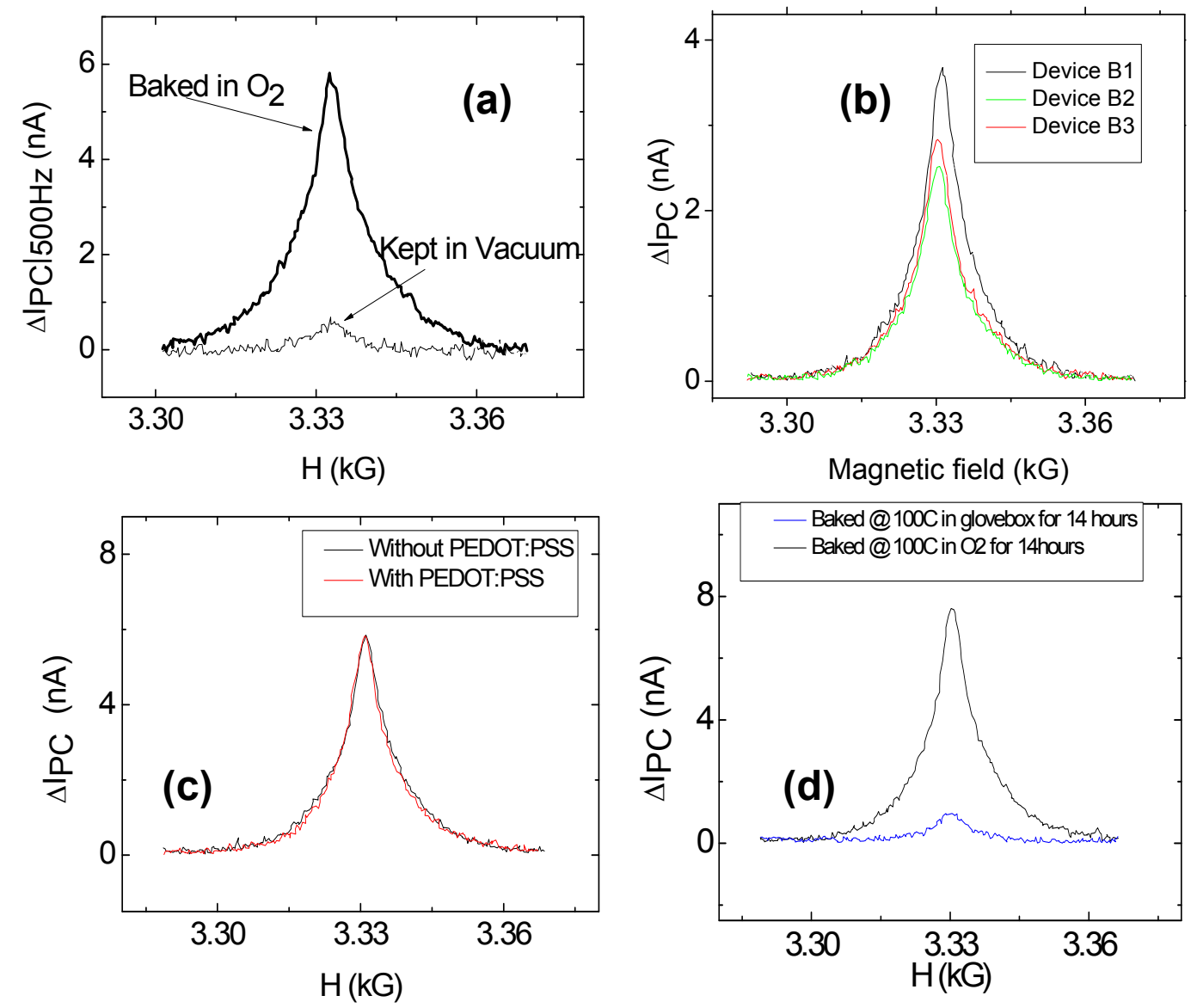

Figure 5.3. (a) $\triangle I_{P C} v s H$ in devices with unoxidized and oxidized MEH-PPV films. (b) $\triangle I_{P C}$ vs $H$ for devices B1-B3, where the MEH-PPV layer was baked in $O_{2}$. Device B1: ITO/MEH-PPV/LiF (Inm)/Al; Device B2: ITO/MEH-PPV/Al; Devices B3: ITO/MoO3 (5nm)/MEH-PPV/Al. (c) $\triangle I_{P C}$ vs H in Device C1 (ITO/PEDOT:PSS/MEH-PPV/Al) and Device C2 (ITO/MEH-PPV/Al), where the MEH-PPV layer was baked in $\mathrm{O}_{2}$. (d) $\Delta I_{P C} v s$ H in devices baked at $100^{\circ} \mathrm{C}$ for 14 hrs in an Ar-filled glove box and in dry oxygen. Device B3 is the reference cell of the batch (i.e., no additional buffer layer beyond the $\left.\mathrm{AlO}_{x}\right)$. We note that $\left|I_{P C}\right|$ under the same excitation conditions was significantly larger in Devices B1 (with the LiF buffer layer) and B2 (with the $\mathrm{MoO}_{x}$ buffer layer) than in Device B3 (the reference device; all values were measured before degradation). Excited at $P_{L}=10 \mathrm{~mW} / \mathrm{mm}^{2}$, Device B1 gives $\left|I_{P C}\right| \sim 1.7 \mu \mathrm{A}$, Device B2 gives $\left|I_{P C}\right| \sim$ 
$3.0 \mu \mathrm{A}$, while Device B3 gives only $\left|I_{P C}\right| \sim 0.4 \mu \mathrm{A}$, indicating obvious differences in carrier transport. Yet as shown in Figure 5.3(b), $\Delta I_{P C}$ exhibited no such strong differences. Thus, the observed effect of oxygen doping on $\Delta I_{P C}$ is less likely to be the result of improved carrier transport.

Another effect of baking of MEH-PPV in $\mathrm{O}_{2}$ could be the dissociation of ITO during the baking procedure. Oxygen and indium from ITO are known to diffuse into MEH-PPV.[33,34] On the other hand, inserting a layer of poly(3,4-ethylenedioxythiophene): poly(styrenesulfonate) (PEDOT:PSS) between ITO and the polymer is reported to block such diffusion [34]. To investigate whether the $\mathrm{O}_{2}$ baking effect on $\Delta I_{P C}$ is caused by such diffusion, devices with a PEDOT:PSS layer inserted between ITO and MEH-PPV (C1) were compared to devices without this layer (reference cell, C2). As Figure 5.3(c) shows, the devices yield similar $\Delta I_{P C}$. Thus, the order-of-magnitude difference in $\Delta I_{P C}$ seen in Figure 5.3(a) is unlikely to be due to ITO dissociation. Figure 5.3(d) compares the devices with the MEH-PPV baked at $100^{\circ} \mathrm{C}$ for $14 \mathrm{hrs}$ in an Ar-filled glove box (oxygen level $<5 \mathrm{ppm}$ ) (D1) vs baked in dry oxygen (reference cell, D2). As clearly seen, $\Delta I_{P C}$ of the device baked in $\mathrm{O}_{2}$ is an order of magnitude stronger than that of the device baked in the glove box. This comparison also demonstrates that $\Delta I_{P C}$ is not due to oxygen or indium diffusion from ITO, as that process would result in a similar $\Delta I_{P C}$ in both baked devices.

With the elimination of the mechanisms discussed above, we consider the following mechanism. Thermally stimulated luminescence studies on MEH-PPV films indicated enhanced formation of deep traps $(0.75-0.91 \mathrm{eV})$ near the surface of 
the films induced by exposure to air [36]. Based on a similar effect of air exposure, the mechanism for the PCDMR could be related to formation of such deep traps.

SIMS depth profiles were obtained to investigate the effects of oxidization on the devices; they are shown in Figures 5.4(a) and 5.4(b). The two profiled samples were prepared as those compared in Figure 5.3(a); their $\Delta I_{P C}$ values were similar. As seen in Figures 5.4(a) and 5.4(b), there is a clear rise in the oxygen concentration near the $\mathrm{Al} / \mathrm{MEH}-\mathrm{PPV}$ interface in the oxidized sample. And in the MEH-PPV bulk region (i.e., the region with the strongly increased carbon concentration), the oxygen concentration first decreases and then rises; it is probably due to oxygen of ITO, since it closely follows the indium and tin concentration profiles in this region. The peak observed in the oxygen concentration near the $\mathrm{Al} / \mathrm{MEH}-\mathrm{PPV}$ interface is consistent with our previous assumption: there is more oxygen related deep traps near the interface and less in the bulk region. On the other hand, in the sample that is not intentionally oxidized, this peak near the A1/MEH-PPV interface is much weaker. This is also consistent with our observation that $\Delta I_{P C}$ is greatly reduced if the sample is not intentionally oxidized, as shown in Figure 5.3(a). As the Al cathodes were deposited simultaneously on both devices, the difference in the oxygen peak levels in these two samples is clearly not due to different levels of remnant oxygen in the evaporation chamber. Hence, this oxygen surplus originates from oxygen trapped in MEH-PPV during its oxidation, although we cannot currently determine the oxygen level that is bonded to $\mathrm{Al}$ or to $\mathrm{C}$, or the trapped $\mathrm{O}_{2}$ following $\mathrm{Al}$ deposition.

It has been reported that bipolarons are stabilized by countercharges to form 
trions in PPVs [16]. It is therefore reasonable to assume that the electron traps near the Al/MEH-PPV interface would stabilize the $b p^{++} \mathrm{s}$ to form positively charged trions. The density of these trions would increase under magnetic resonance conditions, as $\left(p^{+}, p^{+}\right)$pairs in the triplet pair configuration would convert to singlet pairs and form spinless $b p^{++}$. Naturally, the concentration profile of these trions would follow the concentration of the deep traps, which is lower in the bulk and higher toward the interface. Meanwhile, since the $p^{+}$s that contribute to the PCDMR are generated by exciton dissociation, the enhanced formation of trions on these trap sites also assists the dissociation of electron-hole pairs, which in turn releases more electrons. Due to the concentration gradient near the interface, these electrons diffuse to the bulk and thus form a dipole layer with the positively charged trions. The resonance-induced transient redistribution of charges in the MEH-PPV layer generates a transient "image current" in the circuit, which is observed as $\Delta I_{P C}$, and is opposite to $I_{P C}$. An illustration of this model is shown in Figure 5.4(c). When the microwave field is turned on, there is little $\Delta I_{P C}$ because the formation of trions and release of electrons is not immediate. When the population of released electrons reaches its maximum, their redistribution causes the maximum $\Delta I_{P C}$. Each electron will be trapped after some time. Thus, when the device approaches the new steady state at the resonance conditions, no net additional electrons are released, i.e., positive charges originating from bipolaron dissociation counterbalance newly released electrons.

Since $\Delta I_{P C}$ is largely determined by the oxygen concentration gradient, it degrades differently from $I_{P C}$. The degradation of $I_{P C}$ can be caused by many factors, such as 
photooxidation of MEH-PPV [37] or trap formation during operation [38]. However, the evolution of the trap density gradient $\partial n(x, t) / \partial x$ can be very different from the evolution of the trap density $n(x, t)$. For example, the gradient can decrease while the total trap density increases by a large, mostly additive, factor. In addition, the
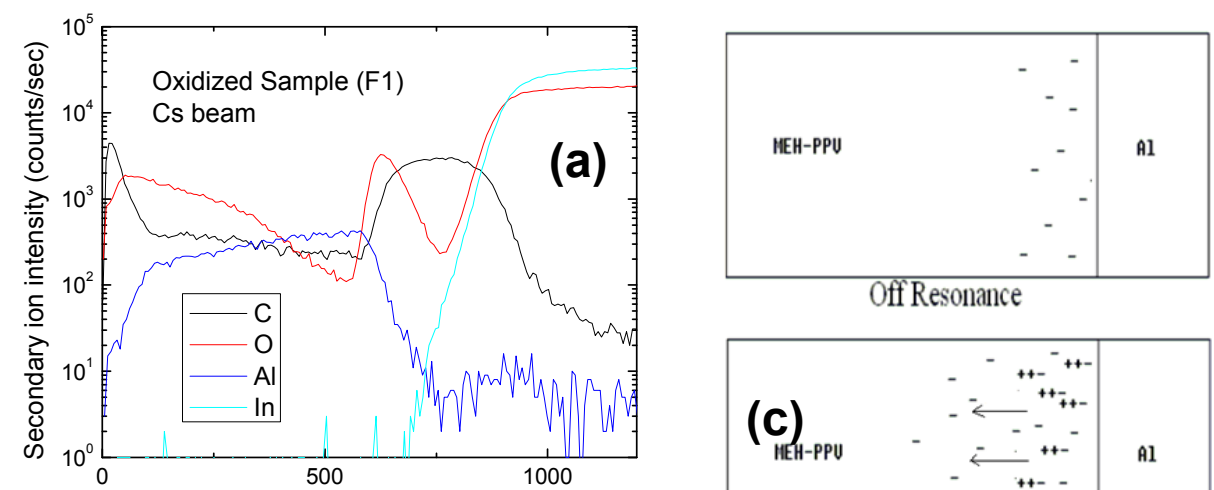

Off Resonance
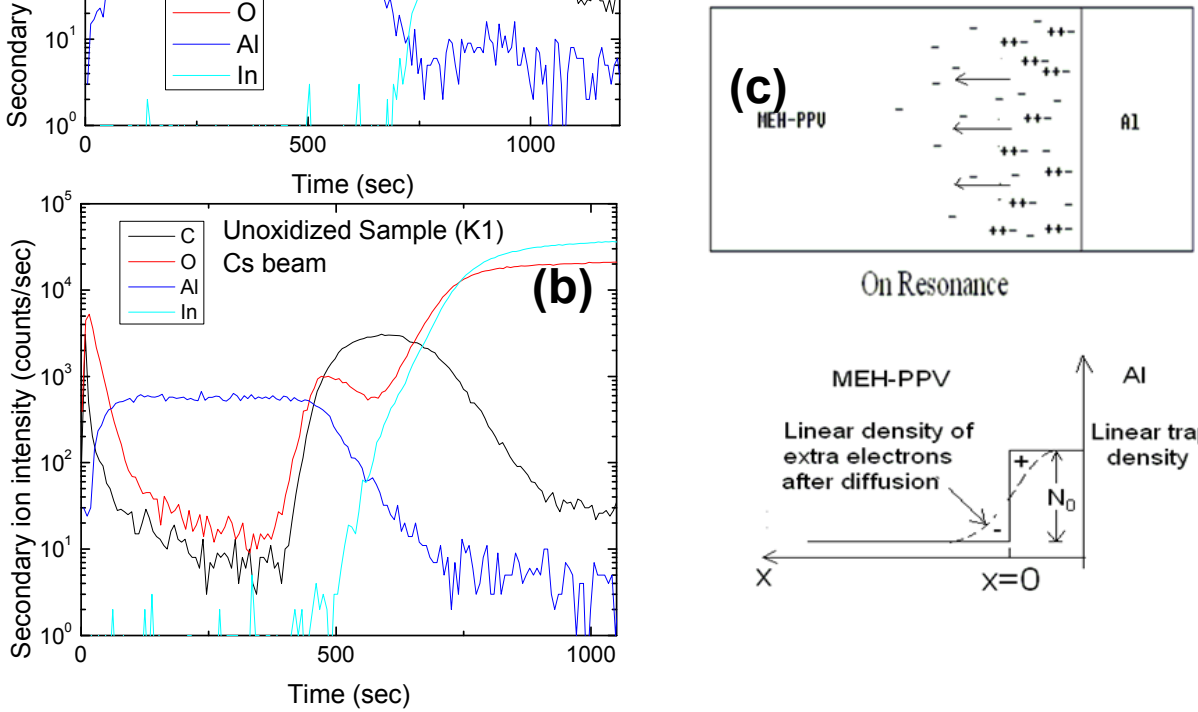

On Resonance

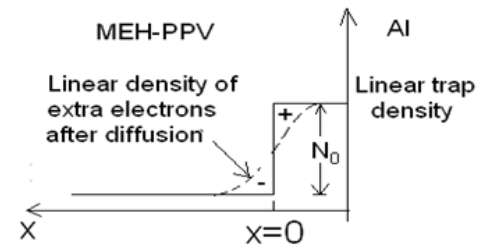

Figure 5.4. (a) and (b) SIMS depth profiles of ITO/MEH-PPV/Al devices with the MEH-PPV layer (a) baked in oxygen and (b) left in vacuum overnight before Al deposition. (c) Suggested PCDMR mechanism: off resonance, traps near the Al/MEH-PPV interface are negative oxygen-related sites. Under resonance conditions, these sites become positively charged by trion formation. Extra electrons are released during the formation of these trions. While trions are pinned at these sites, the diffusion of the released electrons creates an image current in the circuit. 


\subsubsection{A quantitative analysis}

direction of the built-in field would not affect the sign of $\Delta I_{P C}$. Adding a forward bias would therefore further separate the electrons from the trions and lead to a higher $\Delta I_{P C}$, as observed and shown in Figure 5.2.

A quantitative model was developed based on the foregoing scenario. For simplicity, we assume that the trap concentration is a step function; near the $\mathrm{MEH}-\mathrm{PPV} / \mathrm{Al}$ interface the concentration is high and at some point toward the bulk, defined as $x=0$, it drops to a negligible value throughout the bulk region (see Fig. $5.4)$.

Consider the global charge dipole $P(t)$ generated by the magnetic resonance-induced diffusion and trapping of extra electrons. This dipole can be visualized as composed of a fixed layer of positive trions near the Al cathode and the "free" electrons diffusing into the bulk of the MEH-PPV layer. In this scenario,

$$
\Delta I_{P C} \propto d P(t) / d t
$$

Since the extra electrons diffuse throughout the MEH-PPV layer,

$$
P(t)=\int_{0}^{\infty} q x n(x, t) d x
$$

where $q=1.6 \times 10^{-19} \mathrm{C}$ and $n(x, t)$ is the linear density of electrons at position $x$ at time $t$; it is the sum of the linear densities of electrons generated between time $t_{G}$ and $t_{G}+$ $d t_{G}$

$$
n(x, t)=\int_{0}^{t} n^{\prime}\left(x, t, t_{G}\right) d t_{G}
$$

Here the upper bound for $t_{G}$ is $t$ because the generation must have occurred before the detection time $t$. Yet $n^{\prime}\left(x, t, t_{G}\right)$ is governed by two processes: (i) electron generation 
at $t_{G}$ followed by (ii) (re)distribution till $t$, including trapping at some time before $t$.

Hence,

$n^{\prime}\left(x, t, t_{G}\right)=G\left(t_{G}\right) R\left(x, t, t_{G}\right)$

where $G\left(t_{G}\right)$ is the generation rate of extra electrons at $t_{G}$ and $R\left(x, t, t_{G}\right)$ is their normalized distribution function. We consider two possible contributions to $R$ : (i) the contribution $R_{1}$ from electrons that diffuse freely until $t$; (ii) the contribution $R_{2}$ from electrons that become trapped before $t$ (but after $t_{G}$ ). Within this scenario, $R_{1}$ is given by [39]

$R_{1}\left(x, t, t_{G}\right)=r_{f}\left(t^{\prime}\right) \frac{1}{2}\left[1-\operatorname{erf}\left(\frac{x}{\sqrt{4 D_{\text {diff }} t^{\prime}}}\right)\right]$

where $r_{f}\left(t^{\prime}\right)$ is the fraction of these freely diffusing electrons, $D_{\text {diff }}$ is their diffusion constant and $t^{\prime}$ is the diffusion time. Clearly, $t^{\prime}=t-t_{G}$.

For the contribution (ii) from trapped electrons, if the time that the electrons diffused before getting trapped is $s$, we have

$R_{2}\left(t^{\prime}\right)=\int_{0}^{t^{\prime}} r_{t r}(s) \frac{1}{2}\left[1-\operatorname{erf}\left(\frac{x}{\sqrt{4 D_{\text {diff }} s}}\right)\right] d s$

where $r_{t r}(s)$ is the fraction of electrons that become trapped between $s$ and $s+d s$.

We can obtain expressions for $G\left(t_{G}\right), r_{f}\left(t^{\prime}\right)$, and $r_{t r}(s)$ by considering the rate equations for trion generation and trapping of diffusing electrons. The generation of one trion releases two electrons:

$G\left(t_{G}\right)=2 \frac{d n_{\text {trion }}\left(t_{G}\right)}{d t_{G}}$

where $n_{\text {trion }}\left(t_{G}\right)$ is the number of trions at $t_{G}$. We approximate the trion formation rate 
as

$\frac{d n_{\text {trion }}\left(t_{G}\right)}{d t_{G}}=-\frac{d N_{O_{x}^{-}}\left(t_{G}\right)}{d t_{G}}=\frac{N_{O_{x}^{-}}\left(t_{G}\right)}{\tau_{1}}$

where $N_{O x-}\left(t_{G}\right)$ is the number of negatively charged traps that are still available for trion formation at $t_{G}$ and $\tau_{1}$ is the characteristic time of trion formation at those sites.

The solution for $N_{O x-}\left(t_{G}\right)$ is

$N_{O_{\mathrm{x}}^{-}}\left(t_{G}\right)=N_{0} e^{-t_{G} / \tau_{1}}$

where $N_{0}$ is the number of traps at $t_{G}=0$. In this model, it is proportional to the step height at $x=0$. Thus, combining Eqs. (5.8) - (5.10), we have

$G\left(t_{G}\right)=\frac{2 N_{0}}{\tau_{1}} e^{-t_{G} / \tau_{1}}$

Now consider trapping of electrons. Assuming the probability of trapping is the same for every electron, the number of diffusing electrons $n_{\text {diff }}$ is given by

$$
\frac{d n_{d i f f}(s)}{d s}=-\frac{n_{d i f f}(s)}{\tau_{2}}
$$

where $\tau_{2}$ is the lifetime of these diffusing electrons. Therefore, the fraction of these freely diffusing electrons at $s=t^{\prime}$ is

$r_{f}\left(t^{\prime}\right)=e^{-t^{\prime} / \tau_{2}}$

The fraction $r_{t r}(s)$ of electrons that become trapped between $s$ and $s+d s$ is

$r_{t r}(s)=\frac{1}{\tau} e^{-s / \tau_{2}}$

Substituting Eq. (5.13) into Eq. (5.6) and Eq. (5.14) into Eq. (5.7), then those new equations, together with Eq. (5.11), into Eq. (5.5), we get an expression for $n^{\prime}\left(x, t, t_{G}\right)$ 
that is inserted into Eq. (5.4). Then we insert that expression for $n(x, t)$ into Eq. (5.3) to get the following expression for $P(t)$.

$$
\begin{aligned}
& \begin{aligned}
P(t) & =\int_{0}^{\infty} q x \int_{0}^{t} \frac{N_{0}}{\tau_{1}} e^{-\left(t-t^{\prime}\right) / \tau_{1}}\left\{e^{-t^{\prime} / \tau_{2}}\left[1-e r f\left(\frac{x}{\sqrt{4 D_{\text {diff }} t^{\prime}}}\right)\right]+\int_{0}^{t^{\prime}} \frac{e^{-s / \tau_{2}}}{\tau_{2}}\left[1-e r f\left(\frac{x}{\sqrt{4 D_{\text {diff }} s}}\right)\right] d s\right\} d t^{\prime} d x \\
& =\frac{\tau_{2}}{\tau_{1}} N_{0} q D_{\text {diff }}\left(\tau_{1}+\frac{\tau_{1}^{2}}{\tau_{2}-\tau_{1}} e^{-t / \tau_{1}}-\frac{\tau_{1} \tau_{2}}{\tau_{2}-\tau_{1}} e^{-t / \tau_{2}}\right)
\end{aligned} \\
& \text { Thus, } \Delta I_{P C}(t) \propto \frac{d P(t)}{d t}=\frac{N_{0} q D_{\text {diff }}}{\tau_{2}-\tau_{1}}\left(e^{-t / \tau_{2}}-e^{-t / \tau_{1}}\right)
\end{aligned}
$$

Obviously, this is a highly simplified analysis. For example, in modeling the diffusion of electrons, charge drift due to the built-in field and the Coulomb attraction between electrons and positively charged trions are neglected as they should, in first approximation, simply decrease the value of $D_{\text {diff. }}$ Indeed, Figures 5.1(b) and 5.1(d) demonstrate that, with $\tau_{1}=56 \mu \mathrm{s}$ and $\tau_{2}=842 \mu \mathrm{s}$, this simple model is in good agreement with the experimental results.

The value of $\tau_{2} \approx 0.84 \mathrm{~ms}$ is plausible given the low electron mobility $\mu_{e} \sim 10^{-8}$ $\mathrm{cm}^{2} / \mathrm{Vs}$ in MEH-PPV [40], which, using the Einstein relation $D=\mu k T / q$ and $L=$ $(D \tau)^{1 / 2}$, yields a diffusion length $L \sim 5 \mathrm{~nm}$. Note that $D>D_{\text {diff }}$, as the latter is affected by the built-in field and the Coulomb attraction to the trions.

\subsection{Conclusions}

In conclusion, for the first time, a colossal transient change in the photocurrent $\Delta I_{P C}$ due to magnetic resonance conditions is observed in ITO/MEH-PPV/Al devices at room temperature, yielding, in effect, a magnetic resonance-induced switch. This 
transient change is largely independent of the off-resonance photocurrent $I_{P C}$, rendering a colossal $\mathrm{cw} \Delta I_{P C} / I_{P C}$ when $I_{P C}$ is small. The magnitude of this resonance signal is greatly strengthened by baking of the MEH-PPV film in pure dry oxygen. The results suggest that the resonance is due to traps induced by oxygen exposure/baking. A plausible scenario involves enhanced formation of positive trions, i.e., positive bipolarons stabilized by a negatively charged (oxygen-induced) trap site. This results in dissociation of electron-hole pairs with released electrons that diffuse to the bulk, forming a dipole layer with the trions. The transient resonance-induced redistribution of electrons causes a transient "image current" in the circuit, which is observed as a colossal PCDMR. Simulations of this model are in good agreement with the experimental results.

\section{References}

[1] L. S. Swanson et al., Phys. Rev. Lett. (1990) 65, 1140

[2] L. S. Swanson et al., Phys. Rev. B (1991) 44, 10617

[3] X. Wei et al., Phys. Rev. Lett. (1992). 68, 666

[4] E. L. Frankevich et al., Phys. Rev. B, (1992). 46, 9320

[5] L. S. Swanson et al., Phys. Rev. B (1992). 46, 15072

[6] J. Shinar et al., Mol. Cryst. Liq. Cryst. (1994). 256, 691

[7] N. C. Greenham et al., Phys. Rev. B (1996). 53, 13528

[8] W. Graupner et al., Phys. Rev. Lett. (1996). 77, 2033

[9] V. Dyakonov et al., J. Appl. Phys. (1996). 79, 1556

[10] E. J. List et al., Phys. Rev. B (2000). 61, 10807 
[11] E. J. List et al., Phys. Rev. B (2001). 64, 155204-1

[12] E. J. List, Phys. Rev. B (2002). 66, 235203-1

[13] G. Li et al., Phys. Rev. B (2004). 69, 165311-1

[14] M. K. Lee et al., Phys. Rev. Lett. (2005). 94, 137403

[15] M. Segal et al., Phys. Rev. B (2005). 71, 245201-1

[16] A. Kadashchuk et al., Phys. Rev. B (2007). 76, 235205-1

[17] P. A. Bobbert et al., Phys. Rev. Lett. (2007). 99, 216801-1

[18] D. R. McCamey et al., Nat. Mat. (2008), 7, 723; D. R. McCamey et al., Phys. Rev. Lett. (2010), 104, 017601.

[19] T. D. Nguyen et al., Nat. Mat. (2010). 9, 345

[20] J. Behrends et al., Phys. Rev. Lett. (2010). 105, 176601

[21] Z. H. Xiong et al., Nature (2004). 427, 821

[22] S. Pramanik et al., Nature Nanotech. (2007). 2, 216

[23] W. J. M. Naber et al., J. Phys. D (2007). 40, R205

[24] M. Wohlgenannt et al., Nature (London) (2001) 409, 494

[25] M. Wohlgenannt et al., Phys. Rev. Lett., (2002), 88, 197401

[26] C. G. Yang et al., Phys. Rev. Lett. (2007) 99, 157401

[27] G. B. Silva et al., Physica B (2001) 308-310, 1078-1080

[28] Braun D et al., Appl. Phys. Lett., 1991 58(18), 1982-4,

[29] Sariciftci N.S. et al. Appl.Phys.Lett. (1993) 62, 585-587

[30] J. De Ceuster et al., Phys. Rev. B (2001). 64, 195206

[31] K. Demirkan et al., Appl. Phys., (2008) 103, 034505 
[32] F. Li et al., Appl. Phys. Lett., (1997) 70, 1233

[33] J. C. Scott et al., J. Appl. Phys., (1996) 79, 2745,

[34] T. P. Nguyen et al., Synth. Met. (2003) 138113

[35] M. P. De Jong et al, Appl. Phys. Lett., (2000) 77, 14

[36] Kažukauskas V. et al., Appl. Phys. Lett. (2002), 80, 017

[37] D. G. J. Sutherland et al., Appl. Phys. Lett. (1996), 68, 2046

[38] S. Kim et al., J. Soc. Inf. Disp., (2004), 5, 14

[39] J. Crank, The Mathematics of Diffusion (Clarendon Press, Oxford, England, 1975), Chap. 2

[40] H. S. Kang et al., Curr. Appl. Phys., (2001), 1, 443 


\title{
Chapter 6. Evidence for holes beyond the recombination zone \& trions in the electron transport layer of OLEDs
}

\begin{abstract}
.
Recent electroluminescence (EL) detected magnetic resonance (ELDMR) and transient EL studies reveal the presence and role of holes that drift beyond the recombination zone and approach the cathode in small molecular OLEDs with specific materials and structures. In particular, these studies suggest that these holes are responsible for trion (i.e., a bipolaron stabilized by a counterpolaron on an adjacent molecule) formation in the electron transport layer, and may contribute to EL spikes observed at the end of a bias pulse. The significance of these holes to overall OLED performance is discussed.
\end{abstract}

\subsection{Introduction}

Optically and electrically detected magnetic resonance (ODMR and EDMR, respectively) studies of luminescent $\pi$-conjugated materials and organic light-emitting devices (OLEDs) have provided deep insight into the dynamics of singlet excitons (SEs), triplet excitons (TEs), $p^{+}$and $p^{-}$positive and negative polarons, respectively, and bipolarons in these systems.[1-16] Inter alia, the negative photoinduced absorption (PA)-detected magnetic resonance (PADMR) spectra demonstrated unambiguously, in a totally model-independent manner, that the spin 1/2 magnetic resonance conditions decrease the polaron and TE populations (Fig. 1 and refs. 3, 10). 
This result was crucial to the elimination of at least one scenario (enhanced TE-TE annihilation to SEs) as responsible for the positive spin 1/2 PLDMR, which is due to the same mechanism as the negative spin 1/2 PADMR. However, the actual spin-dependent process responsible for this decrease in their population, as well as for the positive spin 1/2 photoluminescence (PL)-, electroluminescence (EL)- and electrically-detected magnetic resonance (PLDMR, ELDMR, and EDMR, respectively) (Fig. 2), has not been settled. Many of the experimental results are consistent with enhanced $p^{+}+p^{-}$recombination to SEs at the expense of TEs, i.e., delayed PL and EL that is enhanced by magnetic resonance conditions [1-4,17-19]. However, very strong evidence points to a completely different mechanism, namely enhanced spin-dependent quenching of TEs by polarons, which obviously reduces the TE population [6-16, 20-21]. Perhaps less obvious is that it also decreases the polaron population, as the (typically trapped) polaron gains the energy of the quenched TE, becomes mobile, and hence recombines after a time shorter than it would without quenching the TE. In this scenario, the magnetic resonance conditions, while reducing the overall polaron population, actually increase the current through the OLED, because trapped polarons that do not contribute to the current off-resonance, do so on-resonance. The increased current results in a positive spin 1/2 EDMR, as observed and shown in Fig. $3[12,13]$ The magnetic resonance conditions yield the positive spin 1/2 PLDMR and ELDMR due to reduced quenching of SEs by the reduced populations of polarons and TEs. 


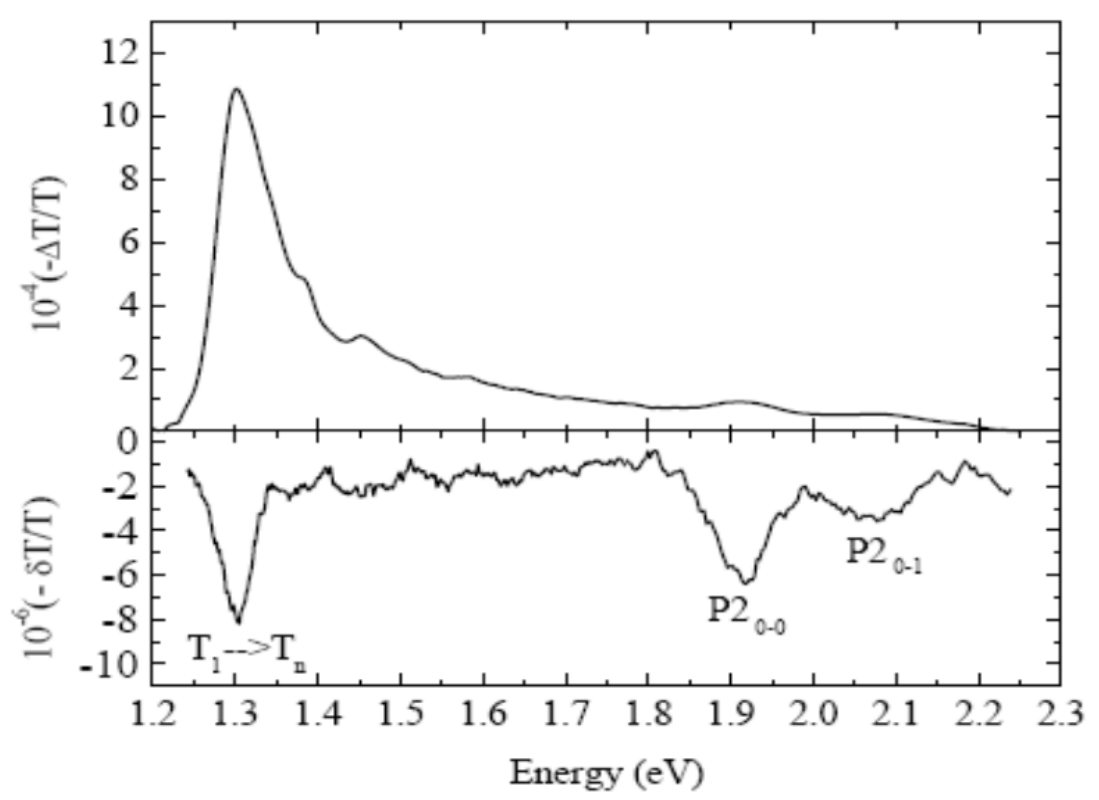

Fig. 6.1. PADMR of methyl-bridged ladder-type poly(p-phenylene) (m-LPPP)[10].

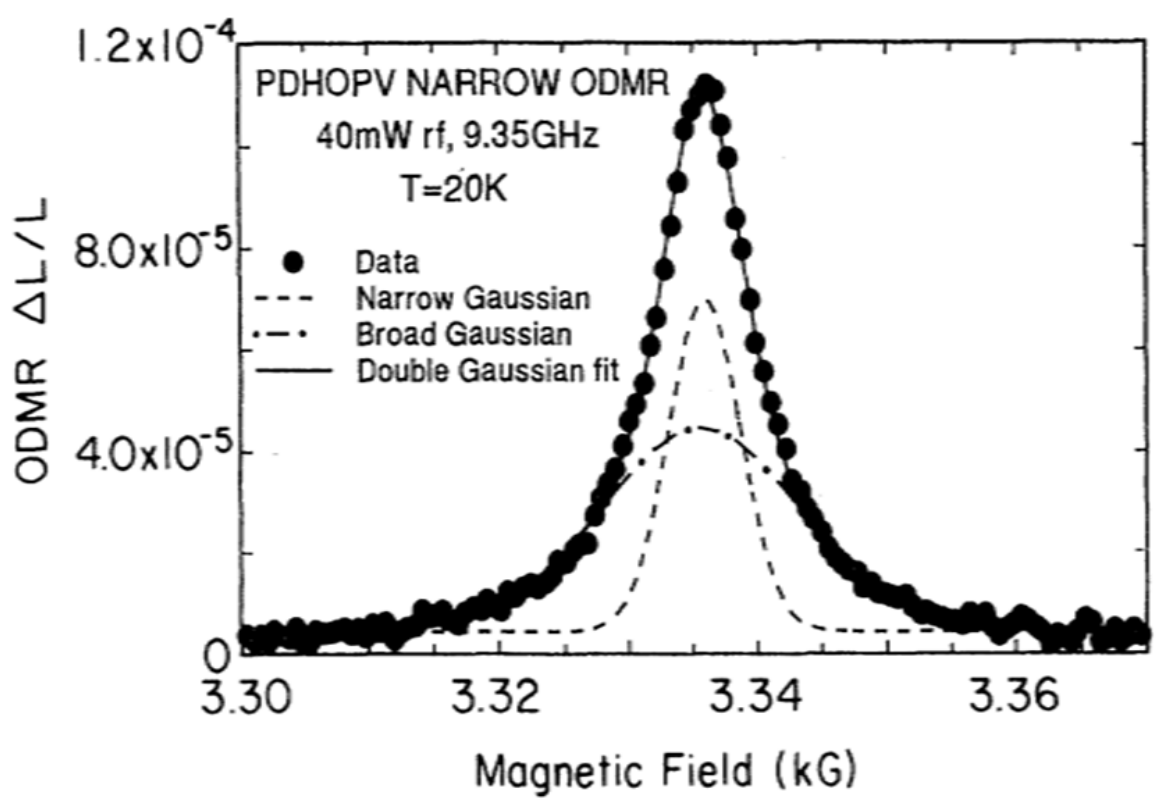

Fig. 6.2. The positive spin 1/2 PLDMR in a film of the dihexoxy derivative of PPV[2].

Besides the negative spin 1/2 PADMR and positive spin 1/2 PLDMR, ELDMR, and EDMR, all of which are clearly due to a common mechanism, luminescent-conjugated materials typically exhibit three other resonances. Two are 
positive TE powder patterns (one around $g=2$ that is $\sim 500-1000 \mathrm{G}$ wide at $\mathrm{X}$ band, the other around $g=4$ that is characteristically asymmetric and $\sim 30 \mathrm{G}$ wide), shown unambiguously to result from reduced quenching of SEs by a TE population that is reduced by magnetic resonance conditions [11]. The third, which is one of the foci of this paper, is a negative spin $1 / 2$ PLDMR observed under photoexcitation at sufficiently short wavelengths [5] and/or sufficiently low wave chopping frequencies[16], and a similar negative ELDMR and EDMR observed in all OLEDs studied to date $[4,6,12,13]$ (and seen in Fig. 3). In contrast to the positive spin $1 / 2$ resonances, in which the amplitude $|\Delta I / I|$ increases with excitation power $P[10,12]$, typically as $P^{1 / 2}$, the amplitude of the negative resonances generally decreases with excitation intensity $I_{E x c}$ (photoexcitation intensity or injected current density) $[4,13,22]$. Since first reported in 1992 [4], this negative spin $1 / 2$ resonance has been attributed to the enhanced spin-dependent formation of bipolarons (spin dependent since the total spin of the bipolaron is 0 ), and this assignment has not been challenged. Subsequent studies of OLEDs, both polymer (PLEDs) and small molecule (SMOLEDs), indicated that in these devices the bipolarons are affected by the organic/organic and organic/cathode interfaces $[6,12,13]$. A PLDMR study of rubrene has shown that in this material they are induced by oxygen centers that dope it [22]. In particular, that study showed that the absolute value of the amplitude of the oxygen-induced negative resonance, $\left|\Delta I_{P L}\right|$, actually increases with excitation intensity $I_{E x c}$, but sublinearly. As $I_{P L} \propto I_{E x c},\left|\Delta I_{P L}\right| / I_{P L}$ decreases with $I_{E x c}$. Thus, it is becoming clear that this enhanced bipolaron formation occurs at specific sites that typically 
become saturated at low $I_{E x c}$, causing $|\Delta I| / I$, whether PL, EL, or current, to decrease with $I_{E x c}$. This behavior, in particular that of the oxygen-doped rubrene, is consistent with the long-held argument that bipolarons require a countercharge, whether a dopant ion or a counterpolaron on an adjacent molecule, for stabilization [16, 23-25]. In the latter case, the resulting composite state comprising the bipolaron and counterpolaron is a (overall singly charged) trion. Such trions are believed to be the source of the negative spin $1 / 2$ PLDMR observed in poly[2-(N-carbazolyl)-5-(2'-ethyl)-hexoxy-1,4-phenylenevinylene] [16], a PPV derivative that yields high-quality PLEDs.
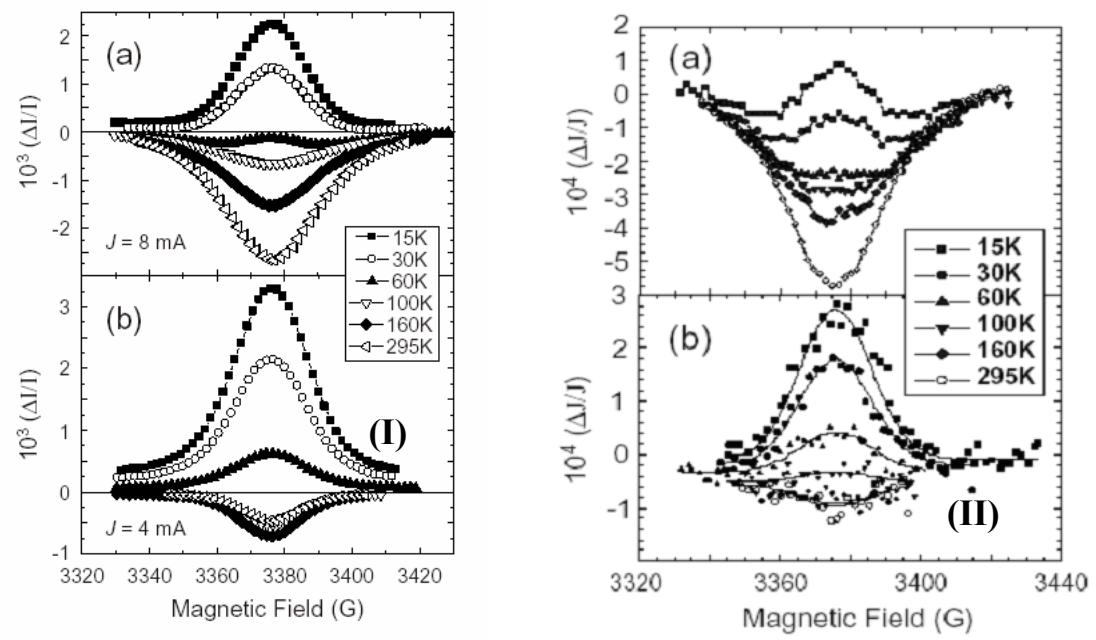

Fig. 6.3. (I) The spin $1 / 2$ ELDMR and (II) spin $1 / 2$ EDMR in (a)

TPD/Alq $3 / \mathrm{AlO}_{x} / \mathrm{Al}$ and (b) TPD/Alq $3 / \mathrm{CsF} / \mathrm{Al}$ OLEDs[12] (see text). Note that the positive spin 1/2 resonances weaken with increasing temperature $T$ while the negative spin 1/2 resonances strengthen with increasing $T$. 
If the negative spin $1 / 2$ resonance is indeed a signature of such trions, which in some cases clearly occur near the organic-cathode interface[12], then there are holes well beyond the recombination zone (RZ) in those cases. This paper examines this scenario by coupling the ODMR and EDMR results with results of transient EL studies that are also consistent with the presence of a significant number of such holes. Such studies yield EL "spikes" or "overshoots" following a bias pulse[26-28], and one mechanism that likely contributes to the amplitude of these spikes is an increased current of holes that "turn back" toward the RZ after having drifted beyond the RZ toward the cathode[28].

\subsection{EXPERIMENTAL}

\subsubsection{ODMR and EDMR measurements.}

The ODMR and EDMR spectra were measured using the system described previously $[1,2,4,6,10,12,13,15,16]$. In brief, the films typically sealed in evacuated $5 \mathrm{~mm}$ outer diameter quartz tubes) and $\sim 3.5 \mathrm{~mm}$ wide OLED samples were placed in a quartz "finger dewar" of an Oxford Instruments He gas flow cryostat, which is inserted in an optically accessible microwave cavity centered between the pole pieces of a dc electromagnet. The resonances were measured by lock-in detection of the changes in the PA, PL, or EL intensity, or current through the OLED, induced by 9.35 GHz microwaves chopped at $500 \mathrm{~Hz}$. The microwaves (typically $160 \mathrm{~mW}$ ) induce a resonance at a magnetic field of $\sim 3.3 \mathrm{kG}$, where the microwave photon energy $h_{w}$ is 
equal to the Zeeman splitting between two spin sublevels

$h_{w}=g \beta H$

where $g$ is the gyromagnetic ratio, $\beta$ is the Bohr magneton, and $H$ is the magnetic field strength. Magnetic resonance changes the spin sub-level populations, which affects the spin-dependent interactions among them.

\subsubsection{OLED Fabrication.}

Indium tin oxide (ITO) / $5 \mathrm{~nm}$ copper phthalocyanine (CuPc) / $50 \mathrm{~nm}$ N,N'-diphenyl-N,N'-bis(3-methylphenyl)-1,1'-biphenyl-4,4'-diamine $\quad$ (TPD) or N,N'-diphenyl-N,N'-bis(1-naphthylphenyl)-1,1'-biphenyl-4,4'-diamine (NPB, $\alpha$-NPB, $\mathrm{NPD}$, or $\alpha$-NPD) / $40 \mathrm{~nm}$ tris(8-hydroxyquinoline) $\mathrm{Al}\left(\mathrm{Alq}_{3}\right) / 1 \mathrm{~nm} \mathrm{CsF} / \sim 120 \mathrm{~nm} \mathrm{Al}$ and other SMOLEDs were fabricated by thermal vacuum evaporation (base pressure $\sim 10^{-6}$ mbar) in a chamber installed in an argon glove box. The $R_{\square} \sim 12 \Omega / \square, 140$ nm-thick ITO-coated glass substrates were etched using $\mathrm{HCl}$ and zinc powder, and cut to $4 \times 20 \mathrm{~mm}^{2}$ for ELDMR and EDMR measurements, or to other desired sizes for other measurements. They were then cleaned as previously described $[29,30]$ using surfactant and acetone, and treated in a UV-ozone oven. Deposition rates were typically $\sim 0.5 \AA / \mathrm{s}$ for the organic layers $\sim 0.1 \AA / \mathrm{s}$ for CsF or LiF buffer layers, and $\sim 5$ $\AA / \mathrm{s}$ for the final Al cathode. It should be noted that significant variations in the ELDMR were observed from batch to batch. Those were probably due to slight variations in the organic/buffer/Al interface, as it is well established that such slight 
variations at the interface can and will significantly affect both the $I-L-V$ curves [31] and the ELDMR and EDMR $[12,13]$. Hence, for ELDMR and EDMR it is only meaningful to compare variations among devices from the same batch, where the organic/cathode interface is as identical in all samples as possible.

\subsubsection{Transient EL measurements.}

The $100 \mathrm{~s}$ bias pulses were generated by an Avtech Model AV-1011 with nominal rise and fall times of $\sim 10$ ns. The EL was monitored by a Hamamatsu R6060-02 photomultiplier tube with a $50 \Omega$ external load resistance connected to a $350 \mathrm{MHz}$ oscilloscope.

\subsection{RESULTS AND DISCUSSION}

\subsubsection{ELDMR.}

Figs. 6.4 and 6.5 shows the effect of adding a layer of 4,7-diphenyl-1,10-phenanthroline (Bphen), a well- known electron-transporting and hole-blocking material [32], between $\mathrm{Alq}_{3}$ and the cathode, to the negative room temperature spin 1/2 ELDMR of $\mathrm{ITO} / \mathrm{CuPc} / \mathrm{NPD} / \mathrm{Alq}_{3} / \mathrm{CsF} / \mathrm{Al}$ OLEDs. As clearly seen, the ELDMR weakens when the BPhen layer is added, consistent with a weaker $h^{+}$(i.e., positive polaron) current and consequent fewer holes that drift beyond the RZ and could stabilize negative bipolarons in either the $\mathrm{Alq}_{3}$ or $\mathrm{BPhen}$ layer that is near the cathode. 


\subsubsection{Transient EL.}

Fig. 6.6(I) shows the transient EL (note the log-log scale) of various $\mathrm{Alq}_{3}$-based SMOLEDs at room temperature. It should be emphasized that at low temperatures, all devices exhibit the spikes at $\sim 70-300 \mathrm{~ns}$ and $\mu$ s-long tails. At room temperature, as seen from Fig. 6.6(I), only those with a hole injection barrier (i.e., no hole injection layer or a $\mathrm{CuPc}$ layer), carrier-trapping guest-host emitting layer (i.e., a doped $\mathrm{Alq}_{3}$ RZ), and no strong hole-blocking layer (i.e., no BPhen) exhibit strong spikes. As Fig. 6.6(II) shows, these narrow and appear earlier under post-pulse reverse bias.

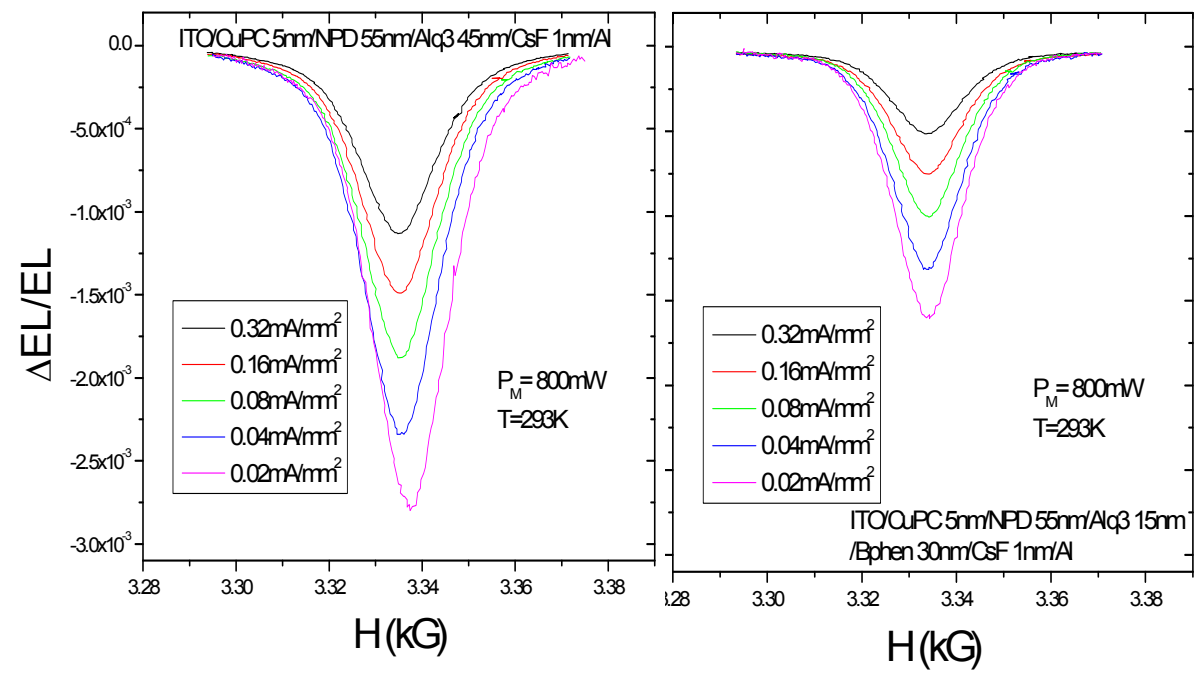

Fig. 6.4. Negative spin 1/2 ELDMR at increasing current density in

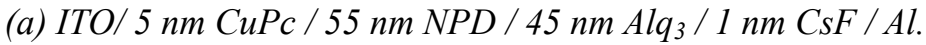

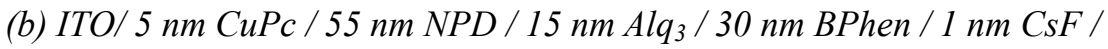

Al. 


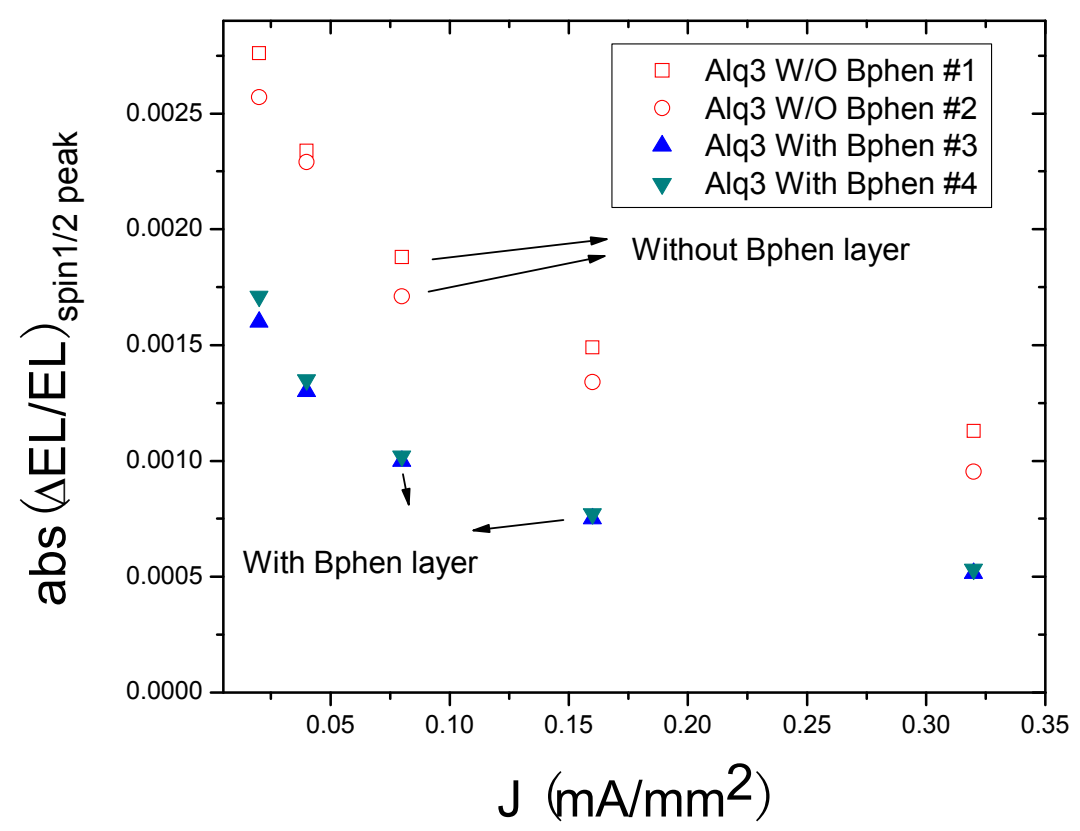

Fig. 6.5. Absolute ELDMR amplitude $\left|\Delta I_{E L} / I_{E L}\right|$ vs current density of two devices without and two devices with a Bphen layer, all fabricated in the same batch As seen from Fig. 6.6(I), the spikes and tails are in good agreement with a model of recombination of correlated charge pairs (CCPs) and initially unpaired charges [28]. However, understanding the occurrence of spikes with amplitudes exceeding the dc EL level is intriguing and challenging. We suspect that decreased post-pulse field-induced dissociative quenching of SEs and CCPs, and possibly some increased post-pulse current of holes that "turn back" toward the RZ after having drifted beyond it cause the intriguing spikes' amplitude. In particular, the effects of the post-pulse reverse bias are qualitatively consistent with a contribution of such holes that drift beyond the RZ toward the cathode [28]. 

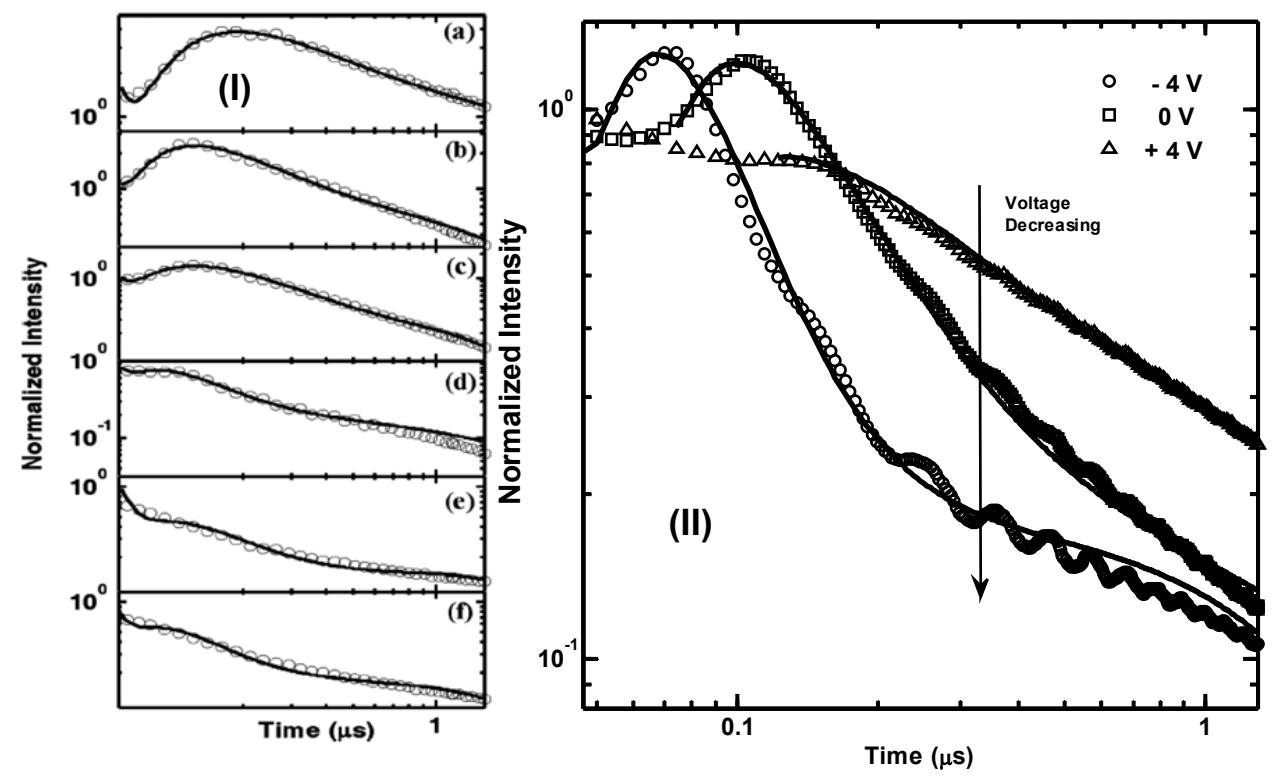

Fig. 6.6. (I) The room-temperature transient EL following a $100 \mu$ s bias pulse for six devices with different structures, normalized to the DC level. The lines are fits by simulations taking recombination of correlated charge pairs (CCPs) and independant charges into account [28].

(a) ITO / $\alpha$-NPB / coumarin6 (C6)-doped Alq 3 / Alq 3 / LiF / Al.

(b) ITO / CuPc / $\alpha-N P B$ / C6-doped $\mathrm{Alq}_{3} / \mathrm{Alq}_{3} / \mathrm{LiF} / \mathrm{Al}$.

(c) ITO / CuPc / $\alpha$-NPB / C6-doped Alq 3 / Alq 3 / BPhen / LiF / Al.

(d) $\mathrm{ITO} / \mathrm{CuPc} / \alpha-\mathrm{NPB} / \mathrm{Alq}_{3} / \mathrm{LiF} / \mathrm{Al}$.

(e) $\mathrm{ITO} / \mathrm{MoO}_{3} / \alpha-\mathrm{NPB} / \mathrm{C6}$-doped $\mathrm{Alq}_{3} / \mathrm{Alq}_{3} / \mathrm{LiF} / \mathrm{Al}$.

(f) $\mathrm{ITO} / \mathrm{MoO}_{3} / \alpha-N P B$ / C6-doped Alq $/ \mathrm{Alq}_{3} / \mathrm{BPhen} / \mathrm{LiF} / \mathrm{Al}$.

(II) Effect of postpulse bias.

Other guest-host SMOLEDs, where the guest clearly traps a hole or electron because its highest occupied molecular orbital (HOMO) level is higher (shallower) 
than that of the host or its lowest unoccupied molecular orbital (LUMO) level is lower (deeper) than that of the host, also exhibit significant spikes at room temperature. These cases, e.g., fac tris(2-phenylpyridine) $\operatorname{Ir}(\operatorname{Ir}(\mathrm{ppy} 3))$-doped spiro-4,4'-Bis(9-carbazolyl) biphenyl (spiro-CBP), which are treated elsewhere [33], confirm the universal nature of this behavior.

\subsection{Conclusions}

In summary, ELDMR, EDMR, and transient EL measurements on various SMOLEDs are consistent with significant generation of negative bipolarons at organic/organic interfaces located between the RZ and the cathode, and near the organic/cathode interface. As it is widely believed that such negative bipolarons require a positive countercharge (on an adjacent molecule) for stabilization, resulting in an overall composite trion with a net single negative charge, it is concluded that the contribution of holes (i.e., positive polarons) that drift beyond the RZ toward the cathode to the stabilization of such negative bipolarons is significant. An increased current of such holes that "turn back" toward the RZ after the bias pulse, and/or reduced quenching of SEs and CCPs by an electric field that decays after the bias pulse, appear to be responsible for the observed EL spikes.

\section{References}

[1] L. S. Swanson et al., Phys. Rev. Lett., (1990), 65, 1140

[2] L. S. Swanson et al. Phys. Rev. B, (1991), 44, 10617 
[3] X. Wei et al., Phys. Rev. Lett., (1992), 68, 666

[4] E. L. Frankevich et al., Phys. Rev. B, (1992), 46, 9320

[5] L. S. Swanson et al., Phys. Rev. B, (1992), 46, 15072

[6] J. Shinar et al., Mol. Cryst. Liq. Cryst., (1994), 256, 691

[7] N. C. Greenham et al., Phys. Rev. B, (1996), 53, 13528

[8] W. Graupner et al., Phys. Rev. Lett., (1996), 77, 2033

[9] V. Dyakonov et al., J. Appl. Phys., (1996), 79, 1556

[10] E. J. List et al., Phys. Rev. B, (2000), 61, 10807

[11] E. J. List et al., Phys. Rev. B, (2001), 64, 155204

[12] E. J. List, Phys. Rev. B, (2002), 66, 235203

[13] G. Li et al., Phys. Rev. B, (2004), 69, 165311

[14] M. K. Lee et al., Phys. Rev. Lett., (2005), 94, 137403

[15] M. Segal et al., Phys. Rev. B, (2005), 71, 245201

[16] A. Kadashchuk et al., Phys. Rev. B, (2007), 76, 235205

[17] P. A. Bobbert et al., Phys. Rev. Lett., (2007), 99, 216801

[18] D. R. McCamey et al., Nat. Mat., (2008), 7, 723; D. R. McCamey et al., Phys. Rev. Lett., (2010), 104, 017601.

[19] T. D. Nguyen et al., Nat. Mat., (2010), 9, 345

[20] J. Behrends et al., Phys. Rev. Lett., (2010), 105, 176601

[21] Z. H. Xiong et al., Nature, (2004), 427, 821

[22] S. Pramanik et al., Nature Nanotech., (2007), 2, 216

[23] W. J. M. Naber et al., J. Phys. D, (2007), 40, R205 
[24] M. Wohlgenannt et al., Nature (London), (2001), 409, 494

[25] M. Wohlgenannt et al., Phys. Rev. Lett., (2002), 88, 197401

[26] C. G. Yang et al., Phys. Rev. Lett., (2007), 99, 157401

[27] G. B. Silva et al., Physica B, (2001), 308-310, 1078

[28] Braun D et al., Appl. Phys. Lett., (1991), 58, 1982,

[29] Sariciftci N.S. et al. Appl.Phys.Lett., (1993), 62, 585

[30] J. De Ceuster et al., Phys. Rev. B, (2001), 64, 195206

[31] K. Demirkan et al., Appl. Phys., (2008), 103, 034505

[32] J. C. Scott et al., J. Appl. Phys., (1996), 79, 2745,

[33] T. P. Nguyen et al., Synth. Met., (2003), 138113

[34] M. P. De Jong et al, Appl. Phys. Lett., (2000), 77, 14

[35] Kažukauskas V. et al., Appl. Phys. Lett., (2002), 80, 017 


\section{Summary and General Conclusion}

In summary, ODMR techniques were used to investigate the dynamics of excitons and charge carriers in $\pi$-conjugated organic semiconductors. Degradation behavior of the negative spin-1/2 ELDMR was observed in Alq3 devices. The increase in the resonance amplitude implies an increasing bipolaron formation during degradation, which might be the result of growth of charge traps in the device. The same behavior of the negative spin-1/2 ELDMR was observed in $2 \mathrm{wt} \%$ Rubrene doped Alq3 devices. However, with increasing injection current, a positive spin-1/2 ELDMR, together with positive spin 1 triplet powder patterns at $\Delta m_{S}= \pm 1$ and $\Delta m_{S}= \pm 2$, emerges. Due to the similarities in the frequency dependences of single and double modulated ELDMR and the PLDMR results in MEH-PPV films, the mechanism for this positive spin-1/2 ELDMR was assigned to enhanced triplet-polaron quenching under resonance conditions. The ELDMR in rubrene doped Alq3 devices provides a path to investigate charge distribution in the device under operational conditions. Combining the results of several devices with different carrier blocking properties and the results from transient EL, it was concluded trions not only exist near buffer layer but also exist in the electron transport layer. This TPQ model can also be used to explain the positive spin-1/2 PLDMR in P3HT films at low temperature and in MEH-PPV films at various temperatures up to room temperature. Through quantitative analysis, TPQ model is shown having the ability to explain most behaviors of the positive spin-1/2 resonance. PCDMR studies on MEH-PPV devices 
revealed a novel transient resonance signal. The signal may originate from the higher concentration of deep traps near cathode. A quantitative analysis based on this assumption was carried out and found to be consistent with the experimental results. 


\section{Acknowledgements}

This work was performed at the Ames Laboratory under contract number DE-AC02-07CH11358 with the U. S. Department of Energy.

I would like to express my deep gratitude to my advisor Dr. Joseph Shinar for all the years' guidance, support and encouragements. Without these, the work presented in this dissertation would not have been possible.

Special thanks to Dr. Ruth Shinar, for all the helpful suggestions and support in my research.

I'm lucky to have all these great collaborators in our group during these years: Gang Li, Moon-Ky Lee, Zhaoqun Zhou, Yun Tian, Min Cai, Yuankun Cai, Zhengqing Gan, Teng Xiao, Rui Liu, Emily Hellerich and Weipan Cui. The assistance and suggestions are very important to me.

Finally, I would like to thank my parents and brother for all these years' selfless love. It was their love that brought me courage to face the challenges. 